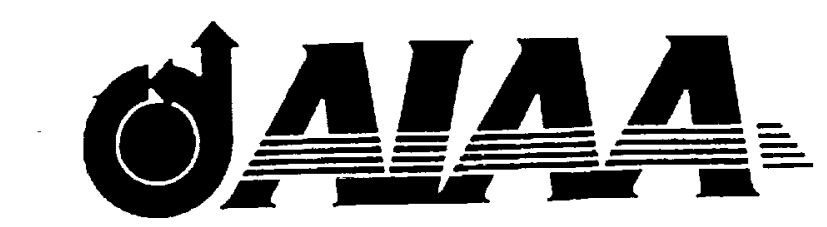

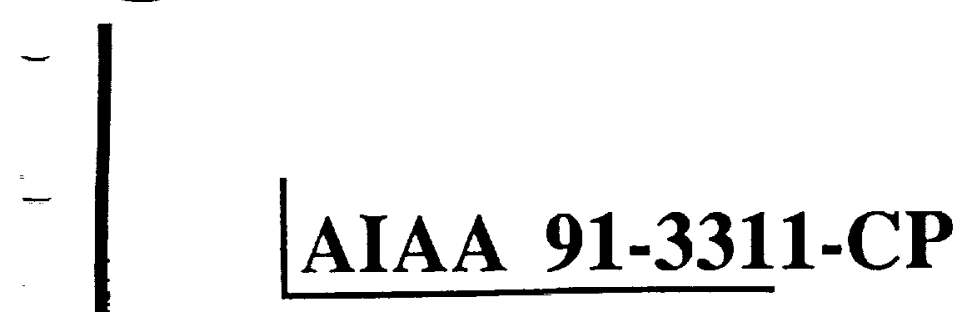 \\ v $91-30083$ \\ SUPERSONIC QUASI-AXISYMMETRIC VORTEX BREAKDOWN
}

Osama A. Kandil and Hamdy A. Kandil Old Dominion University, Norfolk, VA 23529

C. H. Liu

NASA Langley Research Center, Hampton, VA 23665

\begin{abstract}
AIAA 9th Applied Aerodynamics Conference Baltimore, Maryland, September 23-25, 1991
\end{abstract}

For permission to copy or republlsh, contact the American Institute of Aeronautics and Astronautics 370 L'Entant Promenade, S.W., Weshington, D.C. 20024 



\title{
SUPERSONIC QUASI-AXISYMMETRIC VORTEX BREAKDOWN
}

\author{
Osama A. Kandil" and Hamdy A. Kandil"* \\ Old Dominion University, Norfolk, VA 23529 \\ C. H. Liu"* \\ NASA Langley Research Center, Hampton, VA 23665
}

\section{ABSTRACT}

An extensive computational study of supersonic quasiaxisymmetric vortex breakdown in a configured circular duct is presented. The unsteady, compressible, full Navier-Stokes (NS) equations are used for the present computational study. The NS equations are solved for quasi-axisymmetric flows using an implicit, upwind, fluxdifference splitting, finite-volume scheme. The quasiaxisymmetric solutions are time accurate and are obtained by forcing the components of the flowfield vector to be equal on two axial planes, which are in close proximity of each other. The computational study addresses the effect of the Reynolds number, for laminar flows, on the evolution and persistence of vortex breakdown. The effect of boundary conditions at the duct exit on vortex breakdown is also studied. Finally, the effect of swirl ratio at the duct inlet is investigated.

\section{Introduction}

The majority of the experimental studies of vortex breakdown phenomenon has been focused on incompressible flows in pipes ${ }^{1-5}$. Two main types of vortex breakdown have been documented experimentally: the quasiaxisymmetric bubble type and the asymmetric spiral type. Other types of vortex breakdown were also generated in pipes.

The major effort of numerical study of vortex breakdown flows, has also been focused on incompressible, quasi-axisymmetric isolated vortices. Grabowski and Berger ${ }^{6}$ were the first to use the incompressible, quasiaxisymmetric NS equations to study isolated vortex flow in an. unbounded region. Hafez, et. $\mathrm{al}^{7}$ solved the incompressible, steady, quasi-axisymmetric Euler and NS equations using the stream function-vorticity formulation for isolated vortex flows. They predicted vortex breakdown flows similar to those of Garbowski and Berger. Salas and Kuruvila solved the unsteady, quasi-axisymmetric NS equations in a straight circular pipe. They obtained steady, multiple bubble-type vortex breakdown for a Reynolds number range of $100-1,800$. Menne' has also

\footnotetext{
-Professor and Eminent Scholm, Deperment of Mechenical Engineering and Mechinica, Associne Fellow ALA

- Graduate Research Asxistom, Member AIAA.

... Group Leader, Theoretical Flow Physics Branch, Senior Member AIAA.
}

Copyright 1991 by Ouma Kandil. Published by The American Institute of Aeronautics and Astronsurics, line. with permission. used the stream function-vorticity formulation for studying unsteady, incompressible quasi-axisymmetric isolated vortex flows. Wu and Hwang ${ }^{10}$ used the stream functionvorticity formulation to study quasi-axisymmetric vortex breakdown in a pipe. Their study focused on the effects of inflow, wall boundary conditions and Reynolds number on breakdown structure. They showed that the evolution of breakdown can be steady, periodic or unsteady depending on the inflow velocity profiles and Reynolds number. Menne and $\mathrm{Liu}^{11}$ integrated the laminar, incompressible, NS equations for the breakdown of a vortex in a slightly diverging pipe. They showed breakdown flow cases which are based on the purely quasiaxisymmetric and non-axisymmetric analyses. The results were in good agreement with the experimental results of Leibovich4. Spall, et. al ${ }^{12}$ used the vorticityvelocity formulation of the incompressible NS equations to study the three-dimensional vortex breakdown. Breuer and Hänel ${ }^{13}$ solved the unsteady incompressible NS equations using a dual-time stepping, upwind scheme to study the temporal evolution of the three-dimensional vortex breakdown. In Refs. 12 and 13, both types of breakdown; the bubble type and the spiral type, were predicted. Reviews of the physical and computational aspects of the in compressible vortex breakdown were presented by Krause in Refs. 14 and 15. One of the most important aspect of vortex breakdown which Krause discusses in Ref. 15 is the effect of side boundary conditions on the up- or downstream motion of the breakdown point. Also, he presents alternative outflow boundary conditions.

Transonic and supersonic flows around highly swept wings and slender wing-body configurations at moderate to high angles of attack are characterized by interacting vortex cores and shock waves. Other applications which encounter vortex-shock interaction include a supersonic inlet ingesting a vortex and injection of a swirling fuel jet into a supersonic combustor to enhance mixing between the air stream and the fuel ${ }^{16-18}$. In all these applications vortex breakdown due to the interaction of a longitudinal vortex and a shock wave may occur or is intended to occur. For such problems, computational schemes are needed to study, predict and control vortex-shock interaction including vortex breakdown. Unfortunately, the literature lacks this type of analysis with the exception of the preliminary work of Liu, Krause and Menne ${ }^{19}$, Copening and Anderson ${ }^{20}$, Delery, et al ${ }^{16}$, Kandil and Kandil $^{21}$ and Meadows, Kumer and Hussaini ${ }^{22}$. 
$-$

-

$-$

$\underline{\underline{\underline{\underline{\underline{i n}}}}}$

$\overline{1}$

$\bar{\sigma}$

$\underline{\underline{\underline{\underline{\underline{y}}}}}$

$=$

$\bar{\square}$

$\underline{\underline{\underline{\underline{\underline{ }}}}}$

5

$\overline{0}$

晋

$\bar{\Xi}$

$\underline{\underline{\underline{\underline{\underline{\underline{e}}}}}}$

풀

W

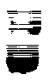

$\bar{\equiv}$ 
The first time-accurate NS solution for a supersonic vortex breakdown was developed by the present authors in Ref. 23. They considered a supersonic quasiaxisymmetric vortex flow in a configured circular duct. The time-accurate solution of the unsteady, compressible NS equations was obtained using an implicit, upwind, flux-difference splitting finite-volume scheme. A shock wave has been generated near the duct inlet and unsteady vortex breakdown has been predicted behind the shock. The predicted flow was characterized by the evolution, convection and shedding of vortex breakdown bubbles. The Euler equations were also used to solve the same problem. The Euler solution showed larger size and number of vortex-breakdown bubbles in comparison with those of the NS solutions. The time-accurate solution was carried out for 3,200 time steps which are equivalent to a dimensionless time of 16 . Only one value of Reynolds number of 10,000 was considered in Ref. 23.

In the present paper, we expand our study of this flow using time-accurate computations of the NS equations with a fine grid in the shock-vortex interaction region and for long computational times. Several issues are addressed in the present study. First, we show the effect of Reynolds number on the temporal evolution and persistence of vortex-breakdown bubbles behind the shock. In this stage of computations, the conditions at the downstream exit are obtained by extrapolating the components of the flowfield vector from the interior cell centers. Although the flow is supersonic over a large portion of the duct exit, subsonic flow exists over a small portion of the exit around the duct centerline. Therefore, selected flow cases have been recomputed using a Riemann-invarienttype boundary conditions at subsonic points of the duct exit. Finally, the effect of swirl ratio at the duct inlet has been investigated.

\section{Formulation}

The conservative form of the dimensionless, unsteady, compressible, full NS equations in terms of timeindependent, body-conformed coordinates $\xi^{1}, \xi^{2}$ and $\xi^{3}$ is given by

$$
\frac{\partial \overline{\mathrm{Q}}}{\partial \mathrm{t}}+\frac{\partial \overline{\mathrm{E}}_{\mathrm{m}}}{\partial \xi^{\mathrm{m}}}-\frac{\partial\left(\overline{\mathrm{E}}_{\mathrm{v}}\right)_{\mathrm{s}}}{\partial \xi^{\mathrm{t}}}=0 ; \mathrm{m}=1-3, \mathrm{~s}=1-3
$$

where

$$
\begin{gathered}
\xi^{\mathrm{m}}=\xi^{\mathrm{m}}\left(\mathrm{x}_{1}, \mathrm{x}_{2}, \mathrm{x}_{3}\right) \\
\overline{\mathrm{Q}}=\frac{\hat{\mathrm{q}}}{\mathrm{J}}=\frac{1}{\mathrm{~J}}\left[\rho, \rho \mathrm{u}_{1}, \rho \mathrm{u}_{2}, \rho \mathrm{u}_{3}, \rho \mathrm{e}\right]^{\mathrm{t}}
\end{gathered}
$$

$$
\begin{aligned}
\overline{\mathrm{E}}_{\mathrm{m}} & \equiv \text { inviscid flux } \\
& =\frac{1}{\mathrm{~J}}\left[\partial_{\mathrm{k}} \xi^{\mathrm{m}} \hat{\mathrm{E}}_{\mathrm{k}}\right]^{\mathrm{l}} \\
& =\frac{1}{\mathrm{~J}}\left[\rho \mathrm{U}_{\mathrm{m}}, \rho \mathrm{u}_{1} \mathrm{U}_{\mathrm{m}}+\partial_{1} \xi^{\mathrm{m}} \mathrm{p}, \rho \mathrm{u}_{2} \mathrm{U}_{\mathrm{m}}\right. \\
& \left.+\partial_{2} \xi^{\mathrm{m}} \mathrm{p}, \rho \mathrm{u}_{3} \mathrm{U}_{\mathrm{m}}+\partial_{3} \xi^{\mathrm{m}} \mathrm{p},(\rho \mathrm{e}+\mathrm{p}) \mathrm{U}_{\mathrm{m}}\right]^{\mathrm{t}}
\end{aligned}
$$

$$
\begin{aligned}
& \left(\overline{\mathrm{E}}_{\mathrm{v}}\right)_{\mathrm{s}} \equiv \text { viscous and beat-conduction flux in } \xi^{\mathrm{s}} \\
& =\frac{1}{\mathrm{j}}\left[0, \partial_{\mathrm{k}} \xi^{\mathbf{8}} \eta_{\mathrm{k} 1}, \partial_{\mathrm{k}} \xi^{\mathbf{3}} \eta_{\mathrm{k} 2}, \partial_{\mathrm{k}} \xi^{\mathbf{3}} \eta_{\mathrm{k} 3}\right. \text {, } \\
& \left.\partial_{k} \xi^{3}\left(u_{n} \tau_{k n}-q_{k}\right)\right]^{i} ; \quad k=1-3, n=1-3 \\
& U_{m}=\partial_{k} \xi^{m} u_{k}
\end{aligned}
$$

The first element of the three momentum elements of Eq. (5) is given by

$$
\begin{aligned}
\partial_{k} \xi^{s} \pi_{x_{1}} & \equiv \frac{M_{x} \mu}{\operatorname{Re}}\left[\left(\partial_{k} \xi^{s} \partial_{1} \xi^{n}-\frac{2}{3} \partial_{1} \xi^{s} \partial_{k} \xi^{n}\right) \frac{\partial u_{k}}{\partial \xi^{n}}\right. \\
& \left.+\partial_{k} \xi^{s} \partial_{k} \xi^{n} \frac{\partial u_{\downarrow}}{\partial \xi^{s}}\right]
\end{aligned}
$$

The second and third elements of the momentum elements are obtained by replacing the subscript 1 , everywhere in Eq. (7), with 2 and 3, respectively. The last element of Eq. (5) is given by

$$
\begin{aligned}
& \partial_{k} \xi^{s}\left(u_{p} \pi_{k p}-q_{k}\right) \equiv \frac{M_{\infty} \mu}{R e}\left[\left(\partial_{k} \xi^{z} \partial_{p} \xi^{n}\right.\right. \\
& \left.-\frac{2}{3} \partial_{\mathrm{p}} \xi^{\mathbf{s}} \partial_{\mathrm{k}} \xi^{\mathrm{n}}\right) \mathrm{u}_{\mathrm{p}} \frac{\partial \mathrm{u}_{\mathrm{k}}}{\partial \xi^{\mathrm{n}}} \\
& +\partial_{k} \xi^{s} \partial_{k} \xi^{n} u_{p} \frac{\partial u_{p}}{\partial \xi^{n}} \\
& \left.+\frac{1}{(\gamma-1) \mathrm{Pr}_{\mathrm{r}}} \partial_{\mathrm{k}} \xi^{*} \frac{\partial\left(\mathrm{a}^{2}\right)}{\partial \xi^{\mathrm{a}}}\right] ; \mathrm{p}=1-3
\end{aligned}
$$

The reference parameters for the dimensionless form of the equations are $L, a_{\infty}, L / a_{\infty}, \rho_{\infty}$ and $\mu_{\infty}$ for the length, velocity, time, density and molecular viscosity, respectively. The Reynolds number is defined as $\mathrm{Re}=$ $\rho_{\infty} V_{\infty} \mathrm{L} / \mu_{\infty}$, where $\mathrm{L}$ is the initial radius of the vortex or the duct inlet radius. The pressure, $p_{\text {, is related }}$ to the total energy per unit mass and density by the gas equation

$$
p=(\gamma-1) \rho\left[e-\frac{1}{2}\left(u_{1}^{2}+u_{2}^{2}+u_{3}^{2}\right)\right]
$$

The viscosity is calculated from the Sutherland law

$$
\mu=\mathrm{T}^{3 / 2}\left(\frac{1+\mathrm{C}}{\mathrm{T}+\mathrm{C}}\right), \mathrm{C}=0.4317
$$

and the Prandel number $P_{r}=0.72$. In Eqs. (1)-(8), the indicial notation is used for convenience. 
$\square$

$\bar{\vdots}$

-

묻

窒

言

를

㖺

害

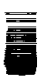

業

E

-

$=$

$\underline{\underline{\underline{\bar{b}}}}$

$\overline{\overline{1}}$

$\bar{\equiv}$

章

5

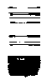

-

$=$

$\overline{\underline{\underline{E}}}$ 


\section{Computational Scheme}

The Computational scheme used to solve the unsteady, compressible full NS equations is an implicit, upwind, flux-difference splitting, finite-volume scheme. It employs the flux-difference splitting scheme of Roe which is based on the solution of the approximate onedimensional Riemann problem in each of the three directions. In the Roe scheme, the inviscid flux difference at the interface of a computational cell is split into left and right flux differences. The splitting is accomplished according to the signs of the eigenvalues of the Roe averaged-Jacobian matrix of the inviscid flux at the cell interface. The smooth limiter is used to eliminate oscillations in the shock region. The viscous and heat-flux terms are linearized and the cross-derivative terms of the viscous Jacobians are dropped in the implicit operator. These terms are differenced using secord-order spatially accurate central differencing. The resulting difference equation is approximately factored and is solved in three sweeps in the $\xi^{1}, \xi^{2}$ and $\xi^{3}$ directions. The scheme is used for third-order spatial accuracy and first-order temporal accuracy. The scheme is coded in the computer program which is called "FTNS3D".

\section{Computational Study and Discussion}

Figure 1 shows a configured circular duct which consists of a short, straight cylindrical part at the inlet which is followed by a short divergent cylindrical part until the axial length of 0.74 . The divergence angle is $6^{\circ}$. The duct radius is then kept constant and a convergent-divergent nozzle with a throat radius of 0.95 is attached. The duct exit radius is 0.98 and its total length is 2.9 . The divergent part of the duct ensures the stability of the formed shock in the inlet region. The configuration of the duct is intended to ensure that the supersonic inflow will become supersonic at the exit. As the computations will show, a small portion of the duct exit near its centerline becomes subsonic at certain times for the specified inflow conditions.

This configured duct has also been used by Delery, et. $\mathrm{al}^{16}$ for their Euler equations computations of supersonic vortex breakdown in an attempt to computationally model an experimental set up. It should be pointed here that the Euler equations, used by Delery, et. al, assume isenthalpic flow in order to drop the energy equation. This is a serious approximation since the upstream flow is rotational. Moreover, as our present calculations show, the flow is actually unsteady and hence, the isenthalpic assumption is not valid.

The NS solver is used to compute all the following flow cases by using a grid of $221 \times 51$ on two axial planes, where 221 points are in the axial direction and 51 points are in the radial direction. In the inlet region up to the 0.74 axial station, 100 grid points are used and the remaining 121 points are used in the remaining part of the duct. The grid is also clustered at the centerline (CL) and the wall. The minimum radial grid size at the $\mathrm{CL}$ is 0.002 . The two axial planes are spaced circumferentially at a certain angle so that the aspect ratio of the minimum grid size will be less than 2 . The upstream Mach number is kept at 1.75 and the Reynolds number is varied from 2,000 up to 100,000 . The initial profile for the tangential velocity is given by

$$
\frac{w}{U_{\infty}}=\frac{k_{e}}{r}\left[1-\exp \left(-\frac{r^{2}}{r_{m}^{2}}\right)\right]
$$

where $U_{x}=1.74, r_{m}=0.2$ and $k_{e}=0.1$. The maximum $\frac{w}{L_{\alpha}}$, swirl ratio $\beta$, is at $r=0.224$. The radial velocity, $v$, at the initial station is set equal to zero and the radial momentum equation is integrated 10 obtain the initial pressure profile. Finally, the density $\rho$ is obtained from the definition of the speed of sound for the inlet flow. With these compatible set of profiles, the computations are carried out accurately in time with $\Delta t=0.0025$. The wall boundary conditions follow the typical NavierStokes solid-boundary conditions. These computations have been carried out on the CRAY YMP of the NASA Langley Research Center. The CPU time is $40 \mu \mathrm{s} / \mathrm{grid}$ pointiteration for the NS calculation.

Next, we present the results of the computational study which covers the effects of Reynolds number, the exit boundary conditions and the inlet swirl ratio.

\section{Effect of Reynolds Number}

For these flow cases, the Reynolds number values are 2,$000 ; 4,000 ; 10,000 ; 20,000$ and 100,000 . The swirl ratio, $\beta$, is kept fixed at 0.32 . The exit boundary conditions are obtained by extrapolation from the interior cell centers.

$\mathrm{Re}=2,000$

Figure 2 shows the streamlines and Mach contours for this flow case at $t=11$, which is equivalent to 4,400 time steps. No vortex breakdown develops and the Mach contours show a steady shock at the duct inlet. The shock is a normal shock over most of the duct inlet. The flow at the duct exit is supersonic.

$\operatorname{Re}=4,000$

Figure 3 shows snapshots of the streamlines and Mach contours for the flow case of $\operatorname{Re}=4,000$. For this value of Reynolds number a single breakdown bubble is seen at $t=5$ and it is convected downstreams as time passes. This breakdown bubble is formed during the downstream motion of the inlet shock, which reaches its maximum downstream displacement at $t=5$. Later on, the shock moves upstream, as it is seen at $t=8$, while the breakdown bubble is convected in the downstream direction. Thereafter, the shock stays stationary at the inlet. This swirling flow case shows a transient single breakdown flow. It should be noticed that at $t=5$ a small portion of the duct exit at its centerline becomes subsonic. At $t=8$, it expands radially to about $25 \%$ of the duct exit radius.

$\operatorname{Re}=10,000$

Figures 4 and 5 show snapshots of the streamlines and 
Mach contours for the flow case of $\operatorname{Re}=10,000$. At $t=3$, a single breakdown bubble is formed behind the downstream moving shock. In this range of $t=3-5$, the bubble grows in all directions while the shock moves downstreams. In the time range of $t=6-8$, the breakdown bubble splits into two bubbles which are convected as they diffuse in the downstream direction. The inlet shock moves upstreams during this time range. During the time range of $t=9-12$, the inlet shock slightly oscillates at the duct inlet while a new breakdown bubble is formed behind the shock. As the bubble size and strength increase, the inlet shock moves very slowly downstreams. More breakdown bubbles (three bubbles) are formed as seen at $t=17$. The breakdown bubbles are then convected downstreams $(l=19)$ while the inlet shock again moves upstreams. As the most downstream bubble is shed through the totally supersonic exit, the inlet shock slowly moves downstreams. In the time range $t=22$ 33 , the shock keeps its slow downstream motion until it becomes stationary. During this time, downstream shedding of breakdown bubbles continues and the formation of new breakdown bubbles slows down until it stops when the inlet shock becomes stationary. Thereafter, no breakdown bubbles are formed and the flow becomes steady. This swirling flow case shows a transient multi-bubble breakdown flow.

$\operatorname{Re}=20,000$

Figures 6 and 7 show snapshots of the streamlines and Mach contours for the flow case of $\operatorname{Re}=20,000$. The mechanism of evolution, convection and shedding of the vortex-breakdown bubbles while the inlet shock is moving downstreams, then upstreams and finally downstreams to become stationary is very similar to the previous case of $\operatorname{Re}=10,000$. However there are some few differences. First, the size, number and strength of breakdown bubbles are larger than those of the case of $R e=10,000$. Second, the displacements of the inlet shock are larger than those of the case of $R e=10,000$. Third, the transient time of the multi-bubble breakdown is longer than that of the case of $\operatorname{Re}=10,000$. The reader can easily compare the snapshots of the streamlines and Mach contours of the two cases at exactly the same time instants. Again this swirling fow case shows a transient multi-bubble breakdown flow.

$\operatorname{Re}=100,000$

Figures 8 and 9 show snapshots of the streamlines and Mach contours for the flow case of $\operatorname{Re}=100,000$. It should be noted that the radial extension of the shown streamlines snapshots is $r=0.6$, which is larger than those of $\operatorname{Re}=2,000-20,000$. Again the mechanism of evolution, convection and shedding of the vortex breakdown bubbles up to $t=30$ is very similar to the previous flow cases of $R e=10,000$ and 20,000 . It is noticed that the size, number and strength of breakdown bubbles are larger than those of the previous cases. Moreover, it is noticed here that short periodic evolution, merging, convection and shedding cycles of the breakdown bubbles occur, e.g.: the time periods of 16-21, 22-27 and 28-32.
At $t=33$ and beyond, a new mode of evolution, convection and shedding of the breakdown bubbles occurs. It should be noticed that the inlet shock keeps on moving slowly in the downstream direction and another shock, which is downstream of the inlet shock, does not extend to the duct axis and is first seen at $t=30$, also keeps on moving in the downstream direction. During the motion of these shocks a breakdown bubble grows behind the inlet shock and sheds a breakdown bubble in the downstream direction. At $t=61$, the downstream bubble grows up in size and strength. Later on, the upstream breakdown bubble also grows up in size and strength. The upstream breakdown bubble becomes larger and stronger than the downstream bubble, and the downstream bubble is convected through the duct exit at $t=78$. Next the upstream breakdown bubble is convected downstreams and new breakdown bubbles appear behind the inlet shock. In the time range of $t=84-95$, the mechanism of the evolution, merging, convection and shedding which is similar to that in the time range of $t=24-35$ is repeated. In the time range of $t=96-120$, the flow is similar to that of the time range of $t=37-78$. At $t=123$ and beyond, the whole process of vortex-breakdown-bubbles evolution, merging, convection and shedding is repeated. It is seen that the snapshots of streamlines at $t=124$ and 130 are exactly similar to those at $t=3$ and 17 . Therefore, it is concluded that the vortex-breakdown mechanism for this flow case is periodic with a long period of time. Within this long cycle, short periodic cycles of vortex-breakdown develops. In summary, this flow case shows that several periodic modes of vortex breakdown develop, which correspond to different frequencies.

\section{Effect of Exit Boundary Conditions}

The Mach contours of the previous cases show that varying small portions of the duct exit become subsonic. Hence, extrapolating the components of the flowfield vector from the interior cell centers for the duct exit boundary conditions is mathematically improper when it is viewed through the behavior of the characteristics at subsonic points. However, such boundary conditions could represent typical physical conditions. Therefore, it is decided to examine the effect of using the Riemann-invariant-type boundary conditions at the subsonic points. This requires that four variables are extrapolated from the interior cell centers while the fifth must be specified at the exit. We chose to specify the pressure at the duct exit at the subsonic points.

For the flow case of $\operatorname{Re}=100,000$, we specified the exit pressure $P_{b}=P_{\infty}$ at the subsonic points. This type of boundary condition is enforced at $t=45$ of the previous flow case (the solution of previous case at $t$ $=45$ serves as initial condition for the present case). Thereafter, the time-accurate integration with this type of boundary condition is continued. Figure 10 shows snapshots of the streamlines and Mach contours of this case. Although the solutions of this case in the time range 
$-$ 
of $t=46-61$ is very similar to those of the previous case, a completely different flow for this case develops as of $t=63$. The two breakdown bubbles which are seen at $t=61$ start moving in the downstream direction. They are continuously convected without any new generation of breakdown bubbles until they are completely shed through the small subsonic portion of the duct exil. In the meantime, the inlet shock disappears and the flow becomes supersonic throughout the duct.

Figures 11 and 12 show the results for $\operatorname{Re}=20,000$ with $\mathrm{P}_{b}=\mathrm{P}_{\propto}$ and $\mathrm{P}_{\mathrm{b}}=2 \mathrm{P}_{\propto}$, respectively. At $\mathrm{t}=5$, the breakdown bubble is exactly the same for these two cases, as it is seen from the streamline figures and the Mach- contour figures as well. At this instant of time, these solutions are the same as those of Fig. 6. It is obvious that the exit boundary conditions has not yet affected the upstream breakdown bubble. At the advanced time instants $t=18-20$, we can see substantial differences between the solutions at each time instant of Figures 11, 12 and 6.

Thus, it is concluded that the exit boundary conditions have a substantial effect on the mechanism of breakdown bubbles evolution, merging, convection and shedding. In particular, the solutions which are based on the Riemann-invariant-type boundary conditions are dependent upon the specified value of the pressure. It should be noted here that the Riemann-invariant-type boundary conditions, as it is well known is one dimensional and is based on inviscid analysis. Although the duct-exit flow is neither one-dimensional nor inviscid, the Riemanninvariant-type-boundary conditions are the best available non-reflective boundary conditions. Therefore, the question remains: what is the proper type of exit boundary condition that one needs to apply? Refer to Krause ${ }^{15}$ for discussion on alternative boundary conditions.

\section{Effect of The Inlet Swirl Ratio}

In this flow case, the Reynolds number is kept at 100,000 and the inlet swirl ratio is increased to 0.38 . Figure 13 shows snapshots of the streamlines and Mach contours up to $\mathrm{t}=30$. Comparing the results of the present flow case with those of $\beta=0.32$, Figs. 8 and 9, we notice that the breakdown bubbles are larger and stronger. Moreover, in the time range of $t=17-30$, the breakdown bubbles are oscillating around a mean position and a process of bubble generation, convection and shedding is taking place around a large breakdown bubble. The inlet shock has a very small amplitude oscillation around a mean position. This is a completely different mechanism from that of the case of Figs. 8 and 9. The computations of this flow case have not yet been carried out further in time.

\section{Concluding Remarks}

The unsteady, compressible NS equations are used for extensive computational study of supersonic quasiaxisymmetric vortex breakdown in a configured cincular duct. The quasi-axisymmetric solutions are time accurate. Several issues have been addressed in this paper. First, we have shown the effect of Reynolds number on the evolution, merging convection and shedding of vortex breakdown bubbles. Several modes of vortex breakdown have been obtained; a transient single-bubble breakdown, a transient multi-bubble breakdown and an unsteady periodic multi-bubble breakdown. These solutions have been obtained by using extrapolated flow conditions from the interior cell centers at the duct exit. Next, selected flow cases have been recomputed using a Riemann-invarianttype boundary conditions at the subsonic points of the duct exit. It has been shown that substantial different solutions have been obtained and the question of what is the proper type of exit boundary conditions remains to be answered. Finally, the effect of the inlet swirl ratio has been investigated for the high Reynolds-number flow. It has been shown that a completely different mechanism of vortex breakdown develops at advanced time instants. Work is underway to understand this mode of vortex breakdown.

\section{Acknowledgment}

For the first two authors, this work has been supported by the NASA Langley Research Center under Grant No. NAG-1-994.

\section{References}

1. Harvey, J. K., "Some Observations of The Vortex Breakdown Phenomenon," Journal of Fluid Mech., Vol. 14, 1962, pp. 595-592.

2. Sarpkaya, T., "Vortex Breakdown in Swirling Conical Flows," AIAA Journal, Vol. 9. No. 9, Sept. 1971, pp. 1792-1799.

3. Sarpkaya, T., "Effect of The Adverse Pressure Gradient on Vortex Breakdown," AIAA Joumal, Vol. 12, No. 12, Dec. 1974, pp. 602-607.

4. Leibovich, S., "Vortex Stability and Breakdown: Survey and Extension," AIAA Journal, Vol. 22, No. 9, Sept. 1984, pp. 1192-1206.

5. Escudier, M. P. and Zender, N., "Vonex Flow Regimes," Joumal of Fluid Mechanics, Vol. 115. 1982, pp. 105-122.

6. Grabowski, W. J. and Berger, S. A., "Solutions of the Navier-Stokes Equations for Vortex Breakdown," Journal of Fluid Mechanics, Vol. 75, Part 3, 1976, pp. 525-544.

7. Hafez, M., Kuruvila, G. and Salas, M. D., "Numerical Study of Vortex Breakdown," Journal of Applied Numerical Mathematics, No. 2, 1987, pp. 291-302. 
8. Salas, M. D. and Kuruvila, G., "Vortex Breakdown Simulation: A Circumspect Study of The Steady, Laminar, Axisymmetric Model," Computers and Fluids, Vol. 17, No. 1, 1989, pp. 247-262.

9. Menne, S., "Vortex Breakdown in an Axisymmetric Flow," AIAA 88-0506, January 1988.

10. Wu, J. C. and Hwang, S., "Computational Study of Vortex Breakdown in Circular Tube," AIAA 91-1820, June 1991.

11. Menne, S. and Liu, C. H., "Numerical Simulation of a Three-Dimensional Vortex Breakdown," Z. Flugwiss. Weltraumforsch. 14, 1990, pp. 301-308.

12. Spall, R. E., Gatski, T. B. and Ash, R. L., "The Structure and Dynamics of Bubble-Type Vortex Breakdown," Proc. R. Soc., London, A429, 1990, pp. 613. 637.

13. Breuer, M. and Hänel, D., "Solution of The 3-D Incompressible Navier-Stokes Equations for the Simulation of Vortex Breakdown," Eight GAMM Conference, Delfh, Netherlands, September 27-29, 1989.

14. Krause, E., "Vortex Breakdown: Physical Issues and Computational Simulation," Third International Congress of Fluid Mechanics, Cairo, Egypt, January 1990, Vol. 1, pp. 335-344.

15. Krause, E.," The Solution of The Problem of Vortex Breakdown," Invited paper, International Conference on Numerical Methods for Fluid Dynamics, Oxford, England, June 1990.
16. Delery, J., Horowitz, E., Leuchter, O. and Solignac, J. L., "Fundamental Studies of Vortex Flows," La Recherche Aérospatiale, No. 1984-2, 1984, pp. 124.

17. Metwally, O., Seutles, G. and Horstman, C., "An Experimental Study of Shock Wave/Vortex Interaction," AIAA 89-0082, January 1989.

18. Cutler, A. D. and Levey, B. S., "Vortex Breakdown in a Supersonic Jet," AIAA 91-1815, June 1991.

19. Liu, C. H., Krause, E. and Menne, S., "Admissible Upstream Conditions for Slender Compressible Vortices," AIAA 86-1093, 1986.

20. Copening, G. and Anderson, J., "Numerical Solutions to Three-Dimensional Shock/Vortex Interaction at Hypersonic Speeds," AIAA 89-0674, January 1989.

21. Kandil, O. A. and Kandil, H. A., "Computation of Compressible Quasi-Axisymmetric Slender Vortex Flow and Breakdown," IMACS 1st International Conference on Computational Physics, University of Colorado, Boulder, June 1990, pp. 46-51. Also in Computer Physics Communications, Vol. 65, NorthHolland, March 1991, pp. 164-172.

22. Meadows, K., Kumar, A. and Hussaini, M., "A Computational Study on the Interaction Between a Vortex and a Shock Wave," AIAA 89-1043, April 1989.

23. Kandil, O. A., Kandil, H. A. and Liu, C. H., "Computation of Steady and Unsteady Compressible QuasiAxisymmetric Vortex Flow and Breakdown," AIAA 91-0752, January, 1991. 
-

$\overline{\overline{\bar{E}}}$

E

$\equiv$

$\overline{5}$

$\overline{\overline{\underline{\underline{\underline{\underline{E}}}}}}$

$\overline{\overline{\underline{\underline{E}}}}$

$=$

三

$\overline{\bar{\equiv}}$

产

$\bar{E}$

$\overline{\bar{\equiv}}$

$\overline{\underline{\underline{\underline{z}}}}$

E

$\overline{\bar{E}}$ 

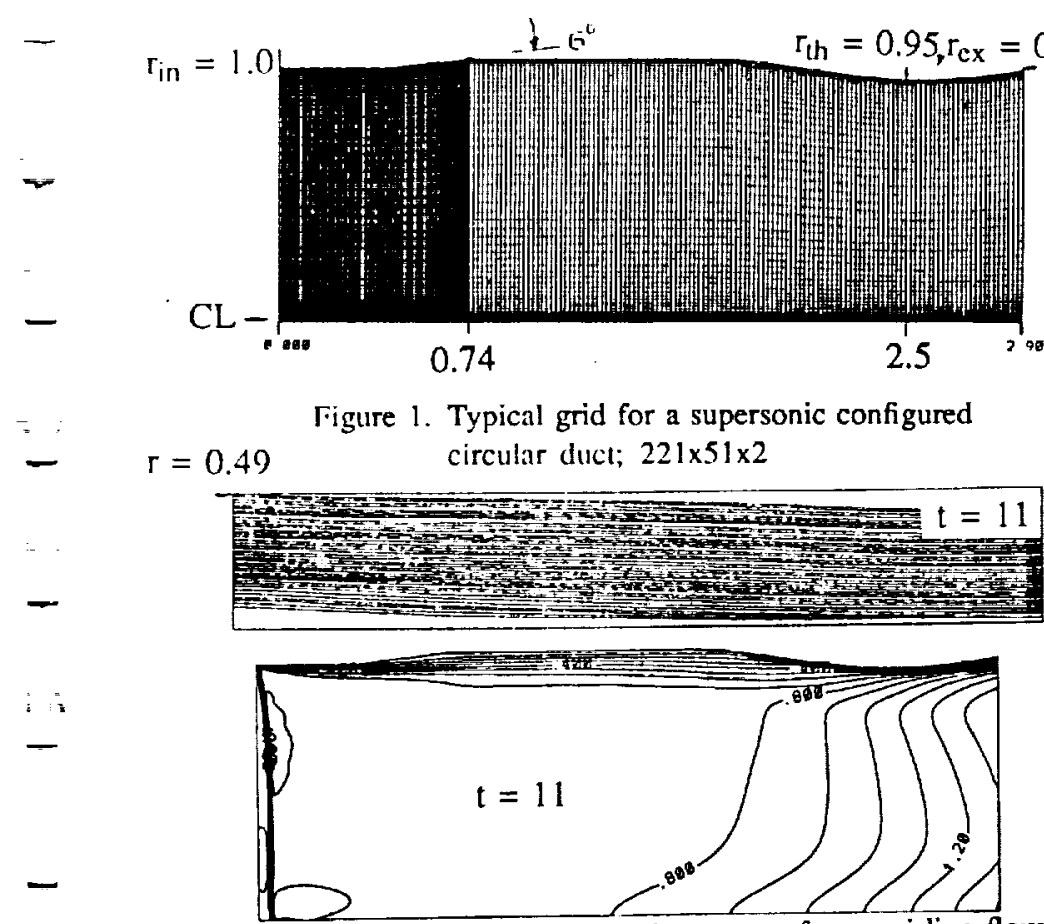

Figure 2. Streamlines and Mach contours for a swirling flow $\begin{array}{ll}r=0.49 & \text { without breakd } \\ R_{\mathrm{i}}=2,000 .\end{array}$

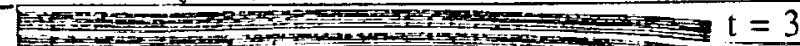

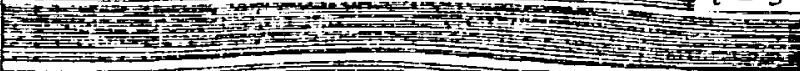
r

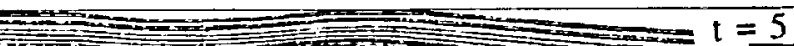

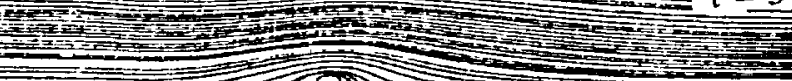

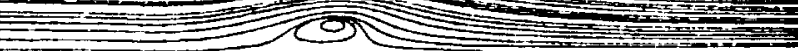

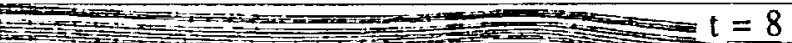
Fin

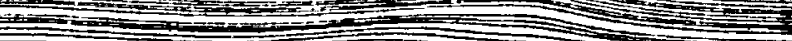
$2=2$

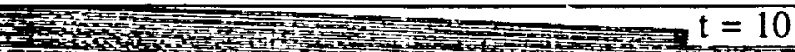

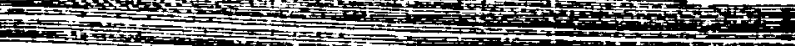
2.
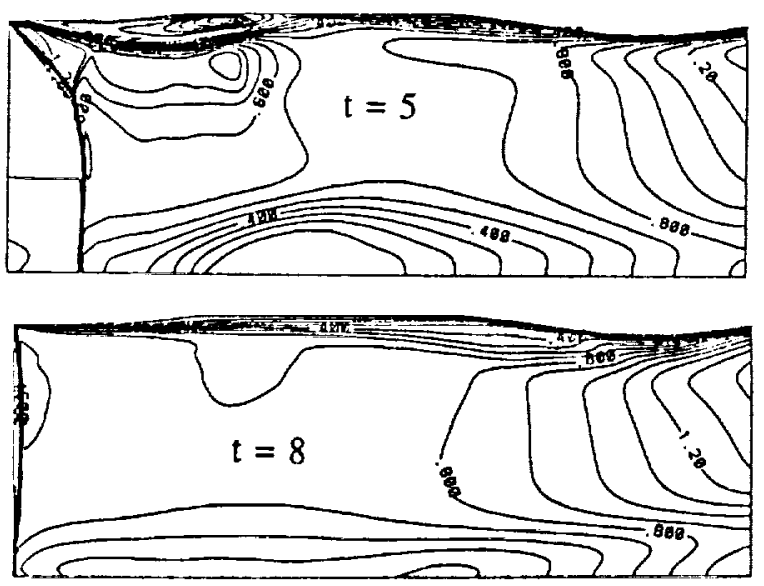

Figure 3. Streamlines and Mach contours for a swirling flow with a transient breakdown, $\mathrm{M}_{\infty}=1.75, \beta=0.32$, $R_{e}=4,000$.
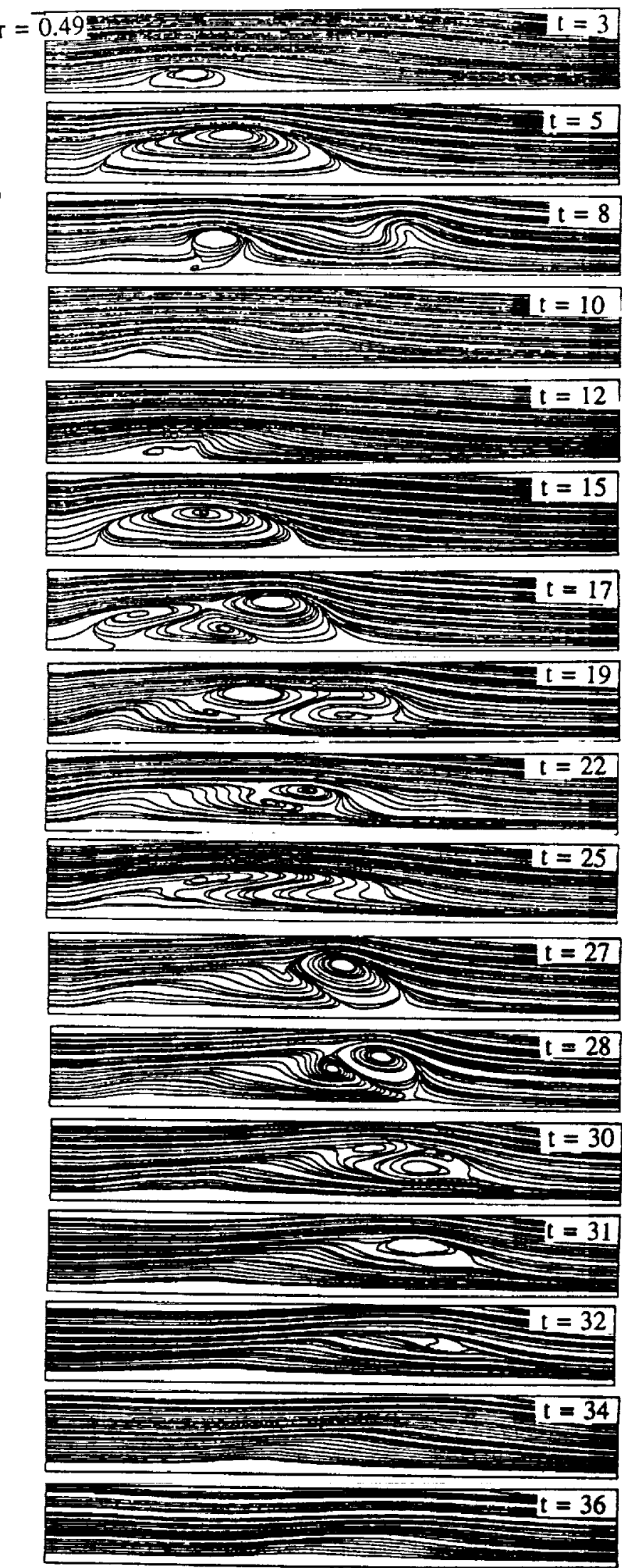

Figure 4. Streamlines for a swirling flow with transient multi-bubble breakdowns, $\mathrm{M}_{\infty}=1.75, \beta=0.32$, $R_{e}=10,000$. 
$\overline{\bar{E}}$

$\bar{\sigma}$

$\overline{=}=$

涪

$\overline{\bar{E}}$

룰

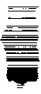

咳

$\overline{\bar{E}}$

$\overline{\bar{E}}$

플

焉

E

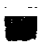

$=$

$\bar{\equiv}$ 

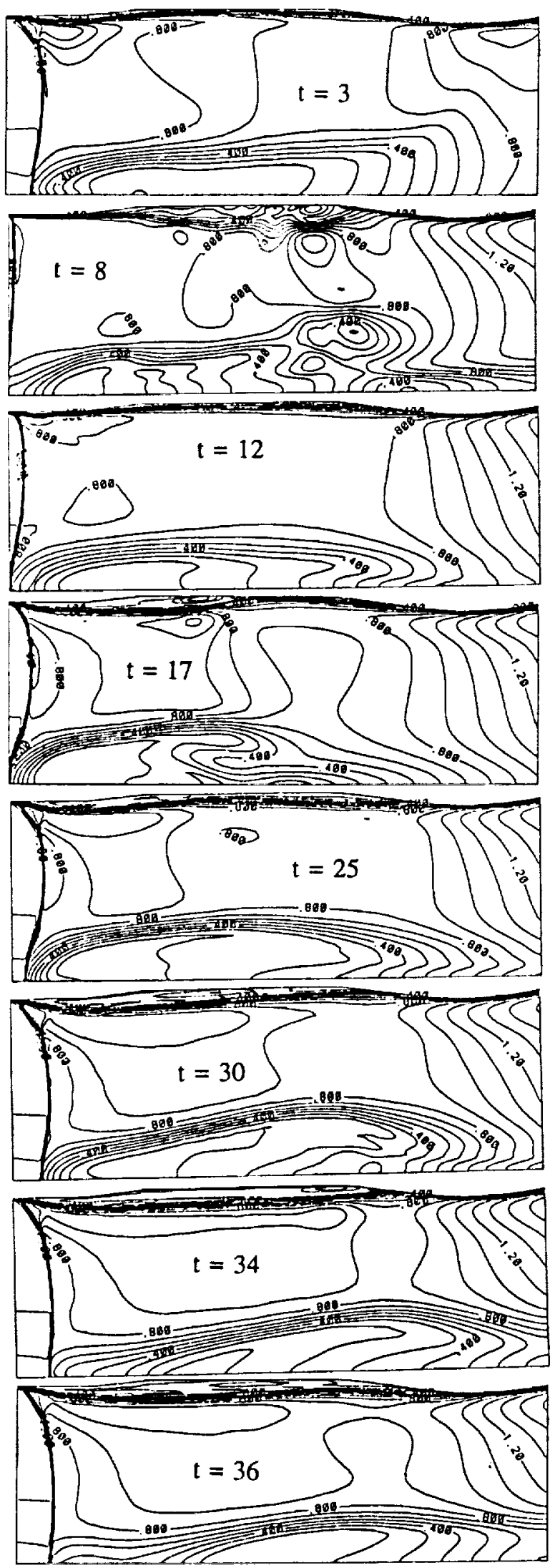

Figure 5. Mach contours for a swirling flow with transient multi-bubble breakdowns, $\mathrm{M}_{\infty}=1.75, \beta=0.32$, $R_{e}=10,000$.
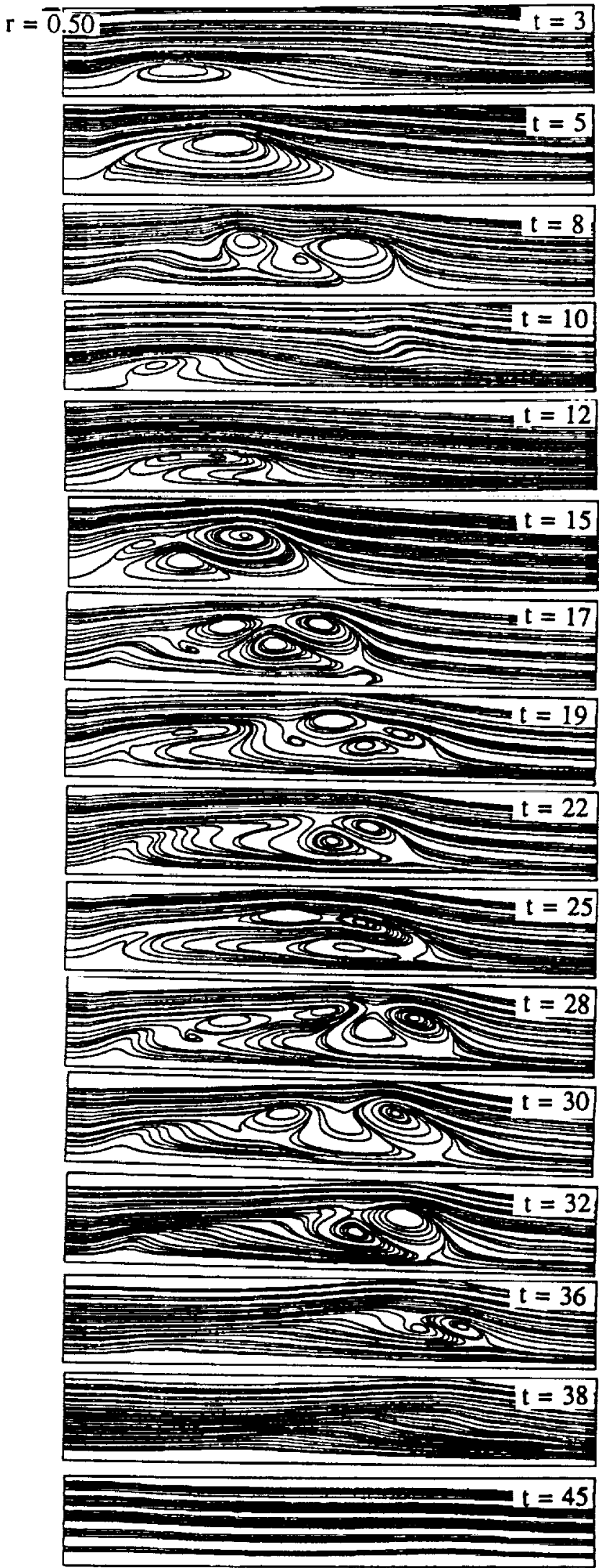

Figure 6. Streamlines for a swirling flow with transient multi-bubble breakdowns, $\mathrm{M}_{\infty}=1.75, \beta=0.32$, $\mathrm{Re}_{\mathrm{e}}=20,000$. 


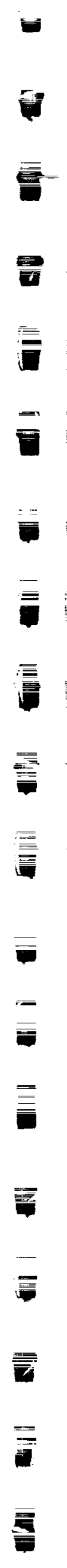



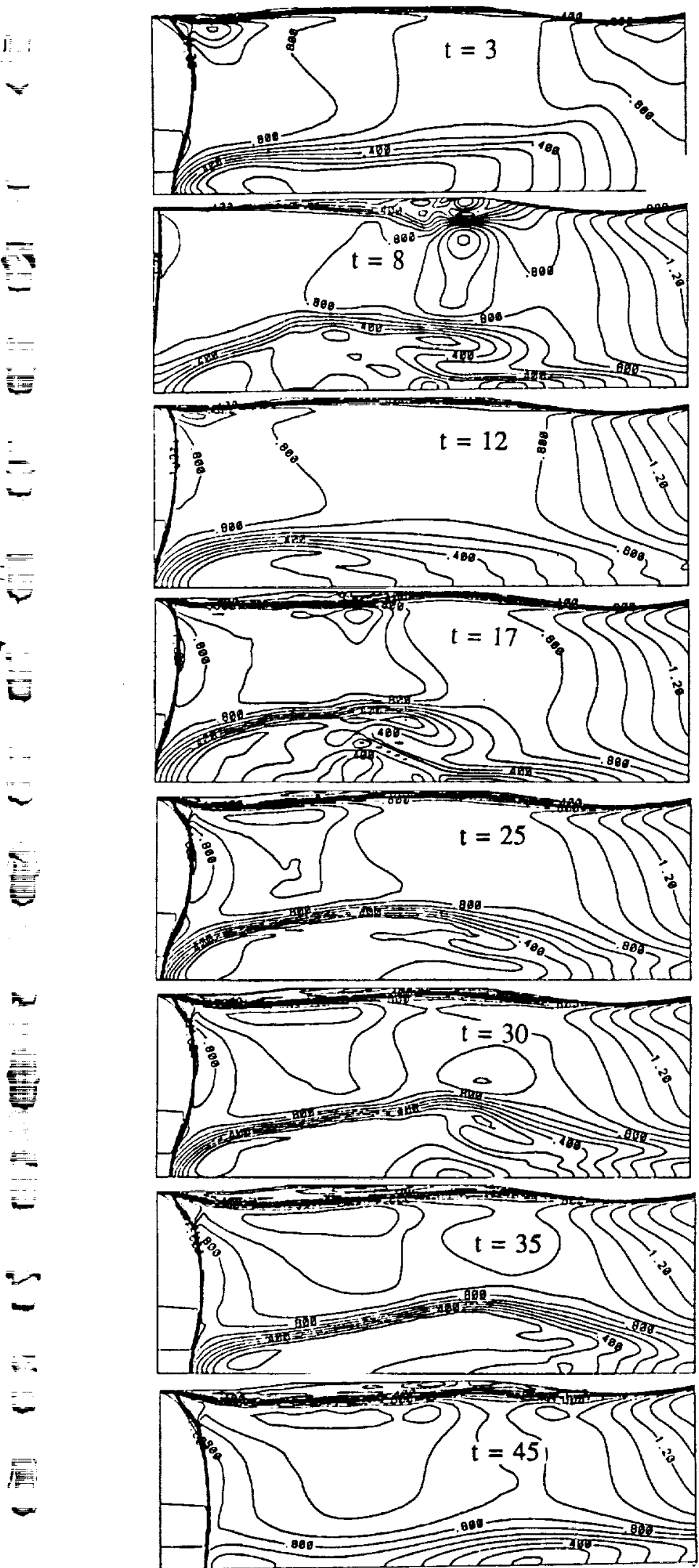

Figure 7. Mach contours for a swirling flow with transient multi-bubble breakdowns, $\mathrm{M}_{\infty}=1.75, \beta=0.32$, $R_{e}=20,000$. $r=0.60 \Longleftrightarrow \mathrm{t}=3$

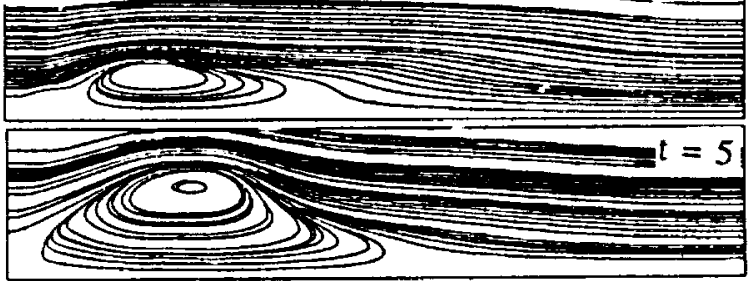

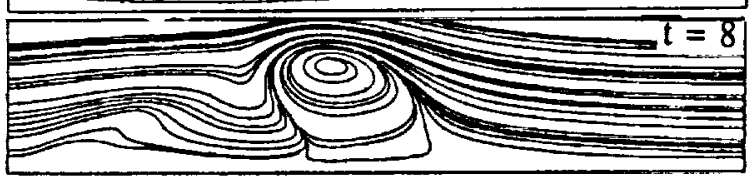
(1)
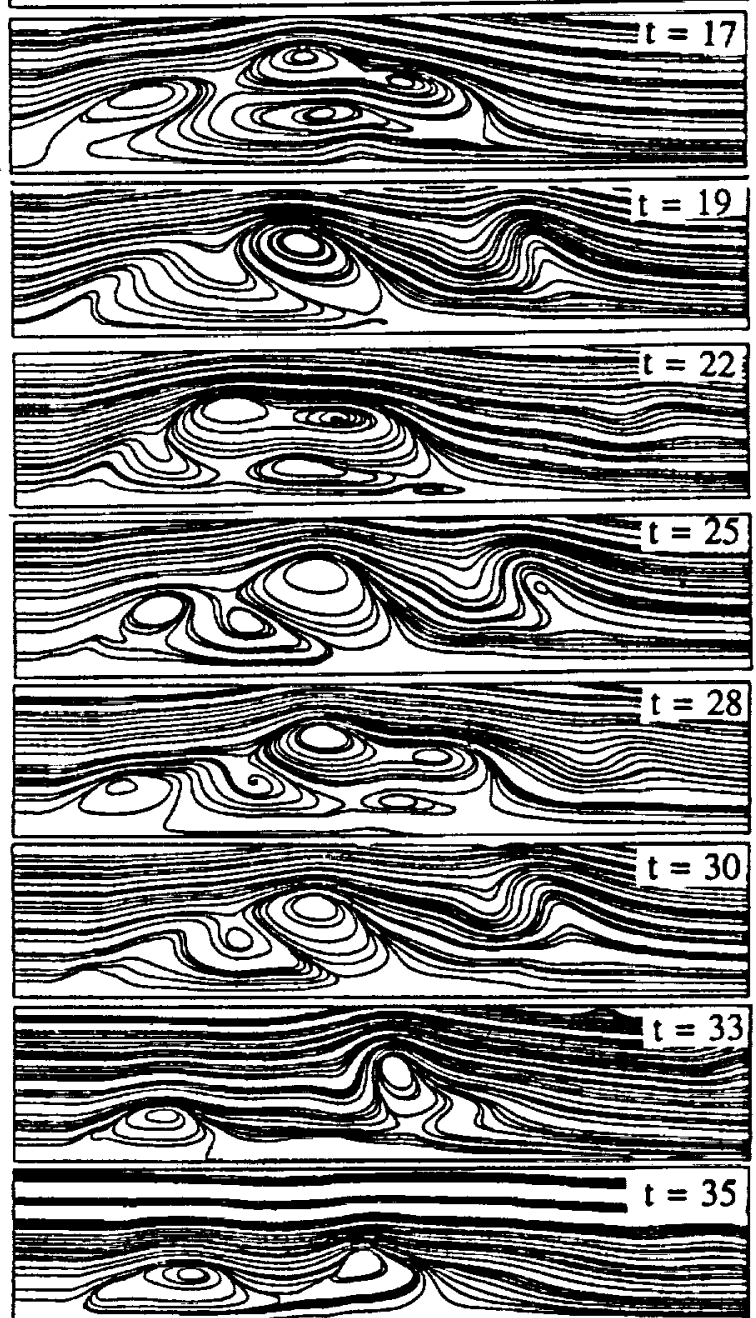


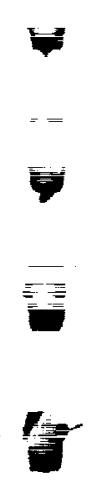

$\overline{0}$

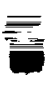

포를

$\varnothing$

$\underline{\underline{\underline{\underline{3}}}}$

$\bar{\equiv}$

$\overline{7}$

言

$-$

$=$

$\Rightarrow$

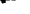

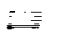

$-$

$\underline{\underline{\underline{\underline{\underline{b}}}}}$

$\overline{\bar{E}}$

$\underline{\bar{E}}$

吾

$\bar{\equiv}$ 
$t=39$ 00 $\mathrm{COm}=2$ 000000010 $\Longrightarrow \mathrm{t}=45$

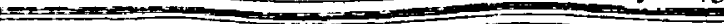

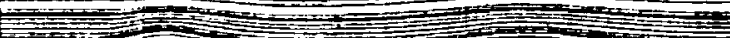
CoSS 2013

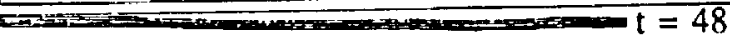
10

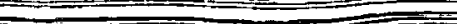

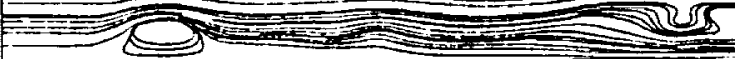
$\Rightarrow t=53$

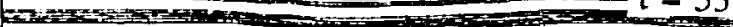

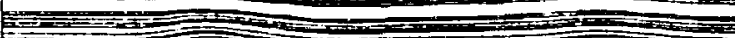
$12 \times 151$ 200005015 $\Rightarrow \mathrm{t}=61$ $\Longrightarrow 1200$

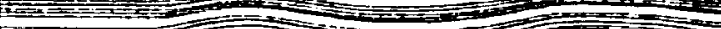
000000005 $\mathrm{t}=66$

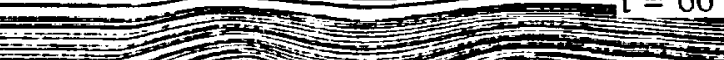

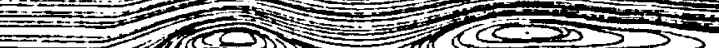
$=0 \times 1025)$
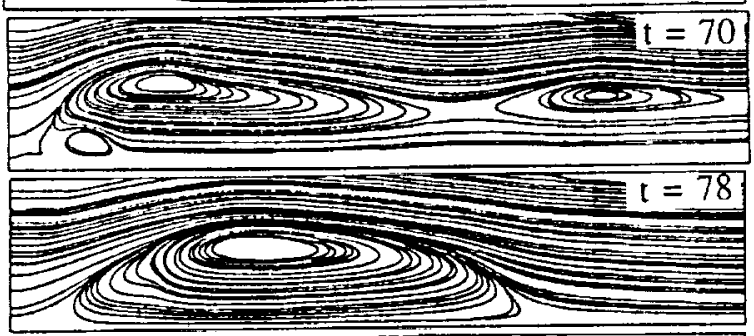
$1 \mathrm{t}=81$

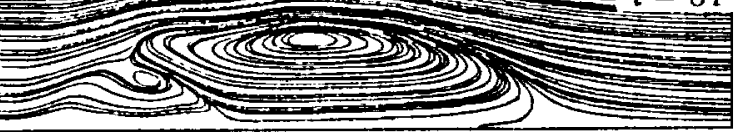
$=0(5) 1$ (ए) and $\mathrm{t}=87$

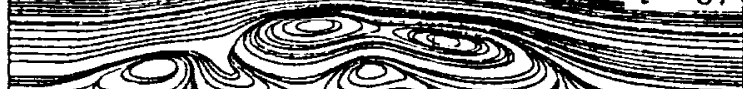

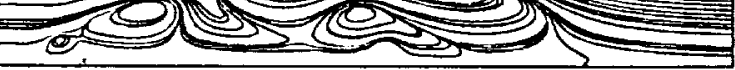

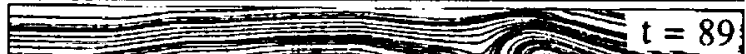

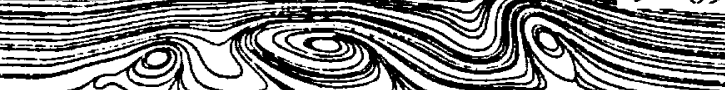
$-150, \ldots$ D $1=93$

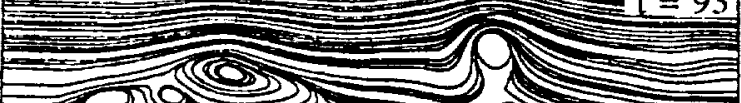

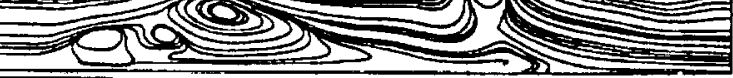
t $=95$

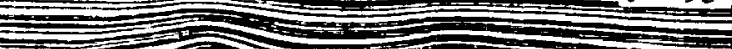

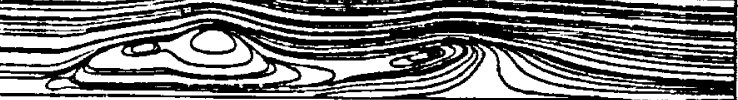
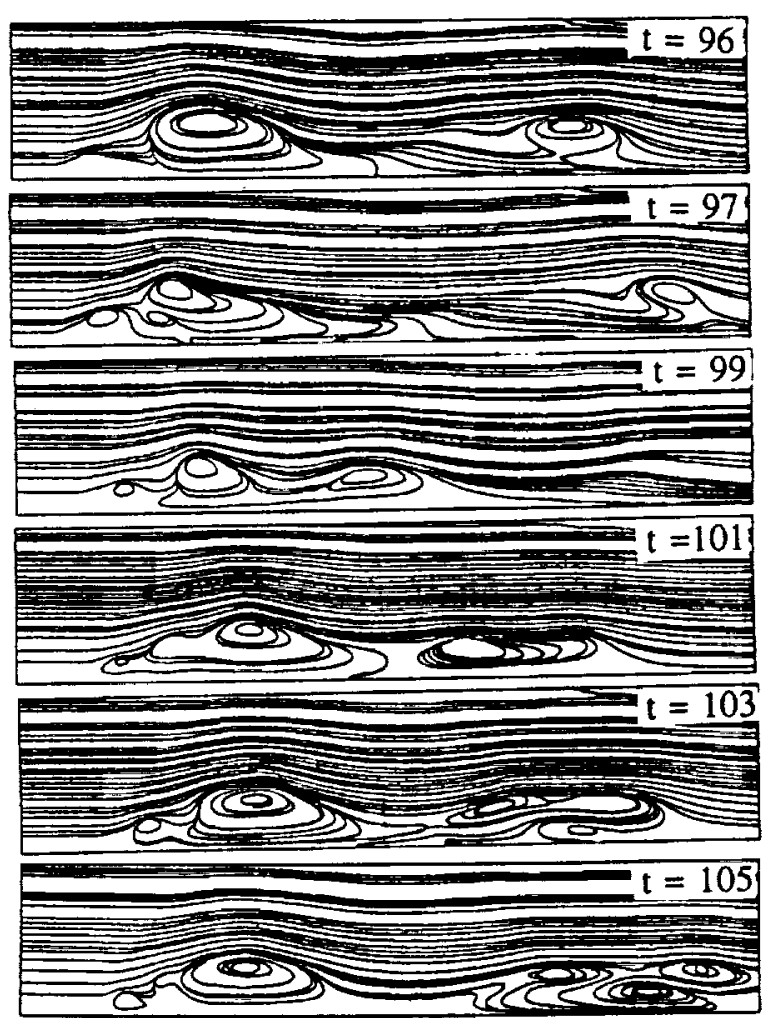

10
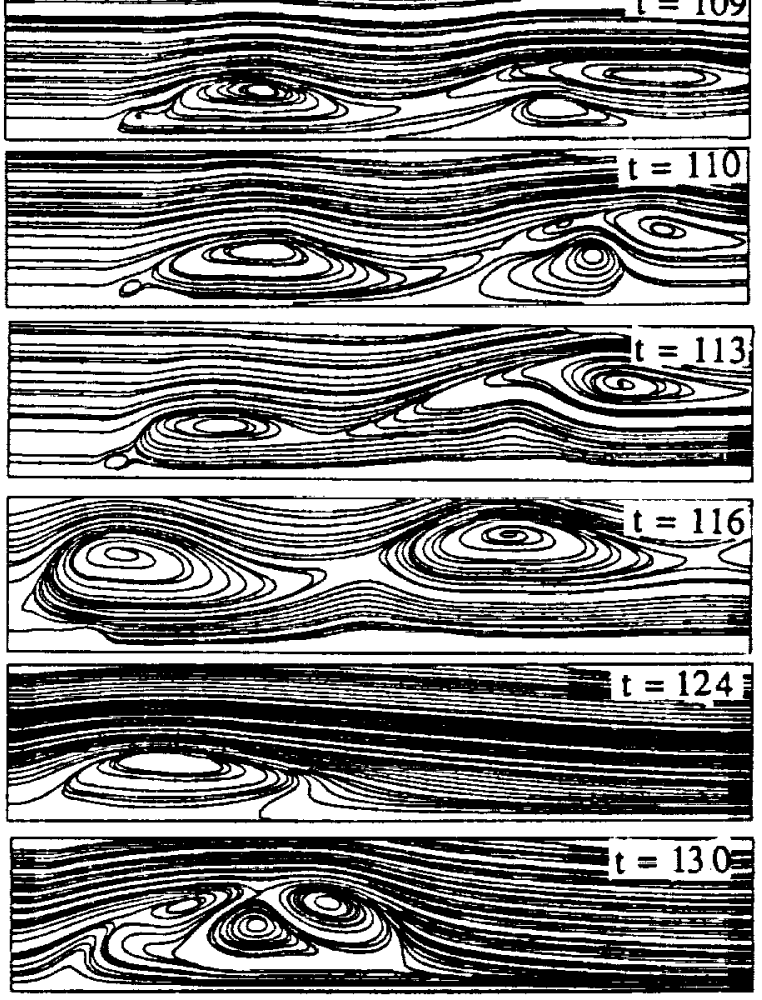

Figure 8. Streamlines for a swirling flow with unsteady multi-bubble breakdowns, $\mathrm{M}_{\infty}=1.75, \beta=0.32$, $R_{e}=100,000$. 


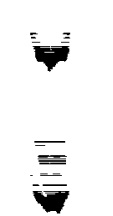

$\overline{\mathbf{z}}$

$\overline{4}$

$=$

汪

些

高

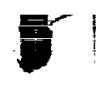

들

$\bar{F}$

衰

$=$

言

$\underline{\underline{\underline{z}}}$

$\overline{\overline{1}}$

$\overline{\bar{E}}$

亨 

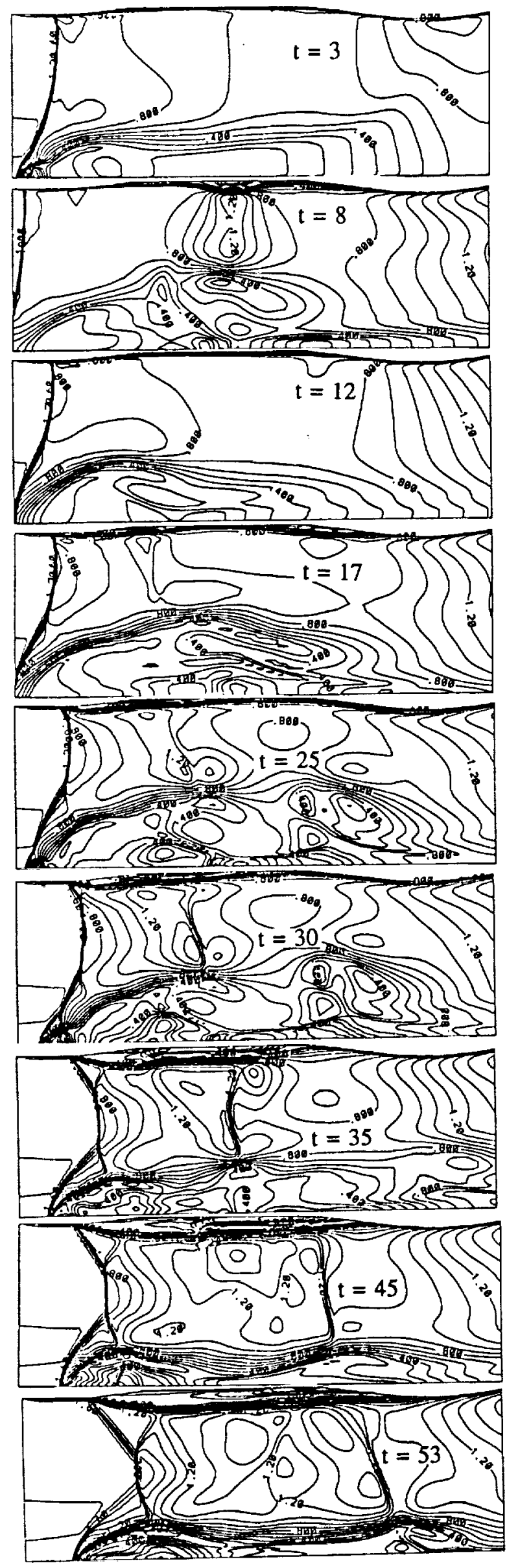
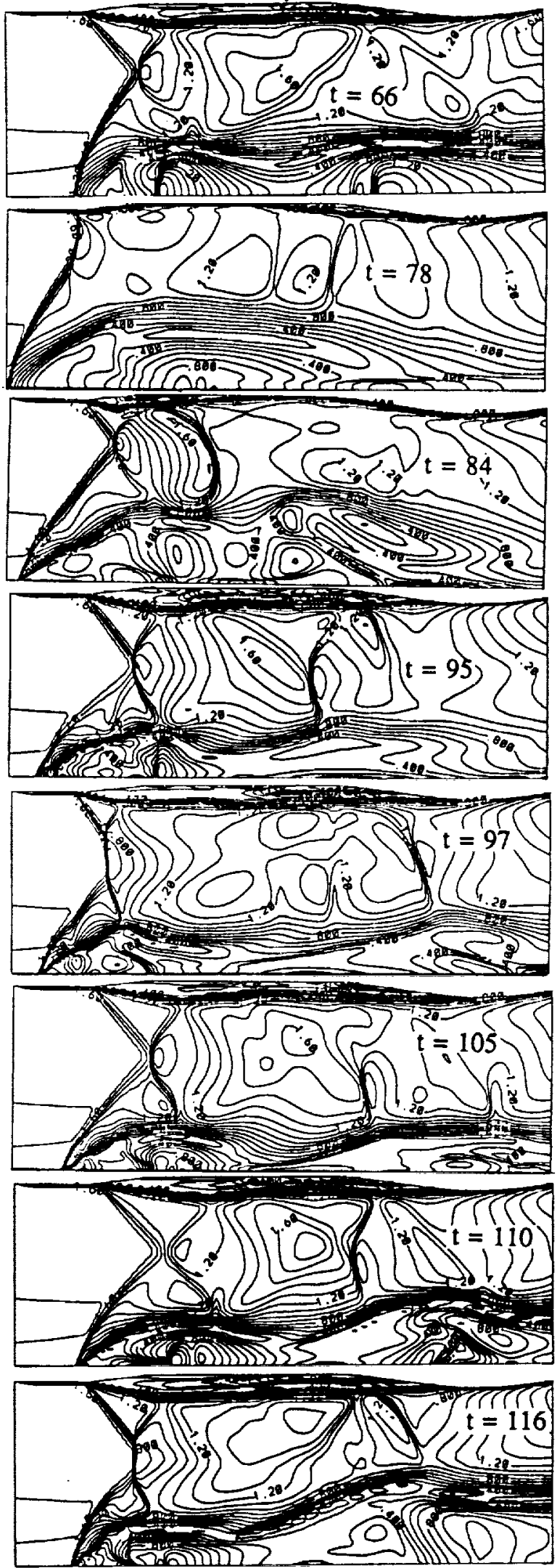

Figure 9. Mach contours for a swirling flow with unsteady multi-bubble breakdowns, $\mathrm{M}_{\infty}=1.75, \beta=0.32$, $\mathrm{R}_{e}=100,000$. 

$\mathrm{r}=0.6 \Longrightarrow \mathrm{t}=53$ 00 20 2035 $t=61$

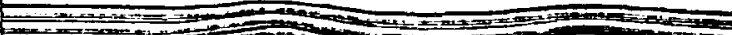

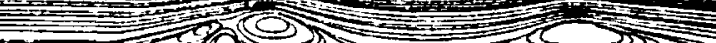
$=2003030203$

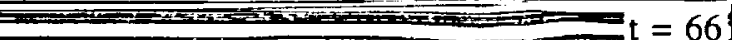
-

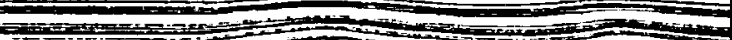
2005000020 $20 \leq 255$
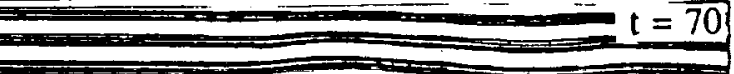

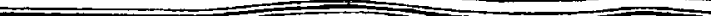
2025
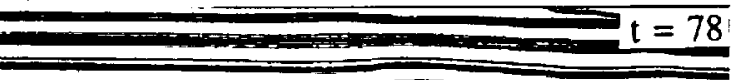
$=$

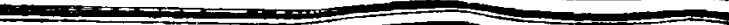
$\longrightarrow 25$

$0 \mathrm{t}=81$

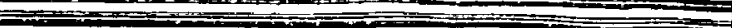

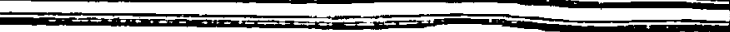

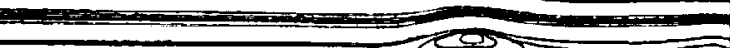
20

$=\mathrm{t}=84$

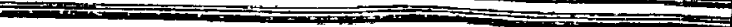
rar $\Rightarrow=0$
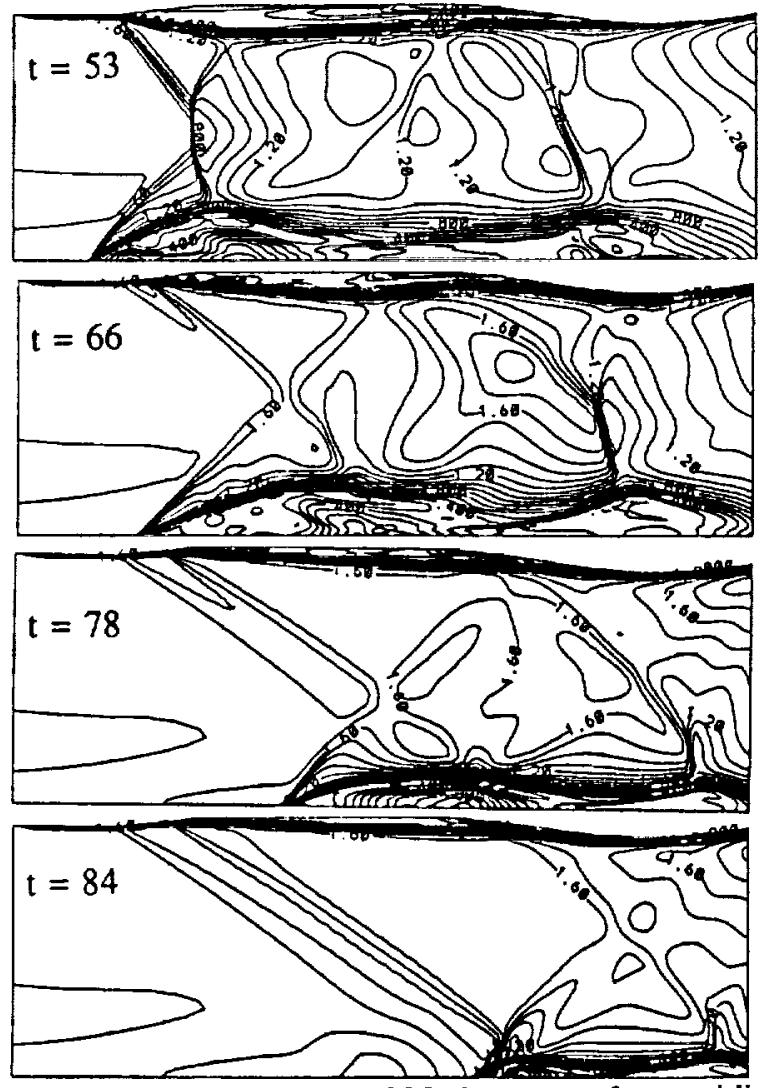

Figure 10. Streamlines and Mach contours for a swirling flow with transient multi-bubble breakdowns, $\mathrm{M}_{\infty}=1.75, \beta=0.32, \mathrm{R}_{e}=100,000, \mathrm{P}_{\mathrm{b}}=\mathrm{P}_{\infty}$
I
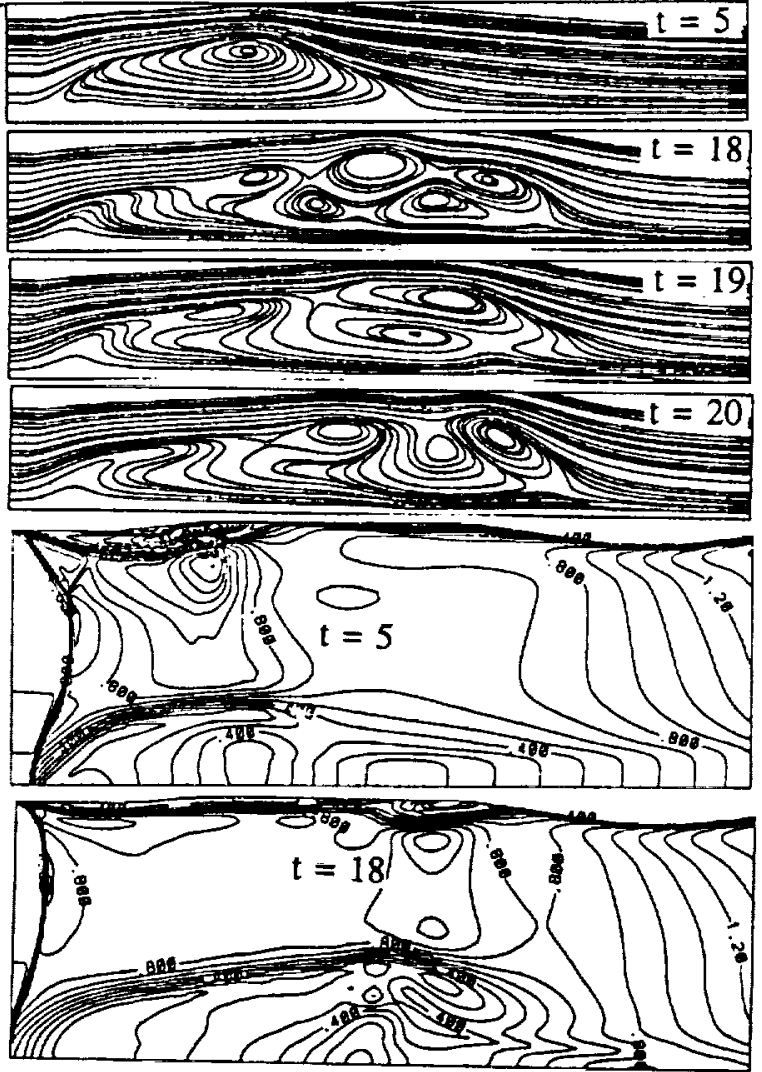

Figure 11. Effect of exit boundary condition, $\mathrm{P}_{\mathrm{b}}=\mathrm{P}_{\infty}$, $\mathrm{M}_{\infty}=1.75, \beta=0.32, \mathrm{R}_{\mathrm{e}}=20,000$.
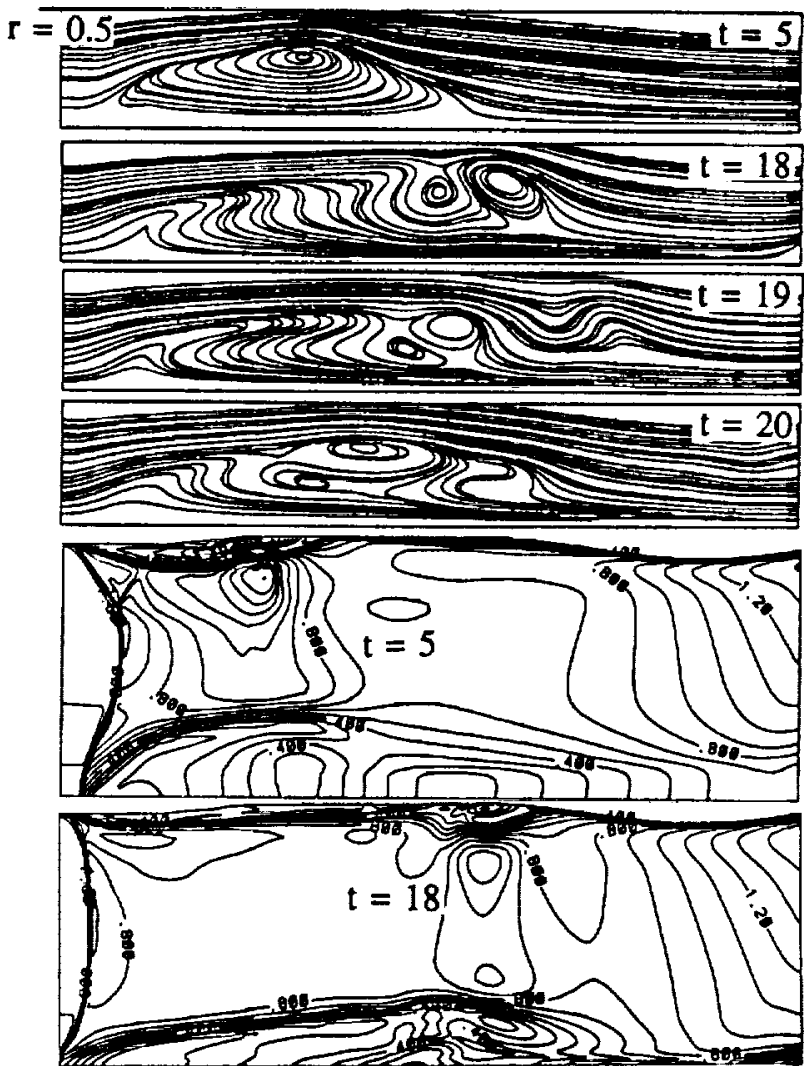

Figure 12. Effect of exit boundary condition, $\mathrm{P}_{\mathrm{b}}=2 \mathrm{P}_{\infty}$ i $\mathrm{M}_{\infty}=1.75, \beta=0.32, \mathrm{R}_{e}=20,000$. 

$\mathrm{r}=0.6 \mathrm{x}=3$

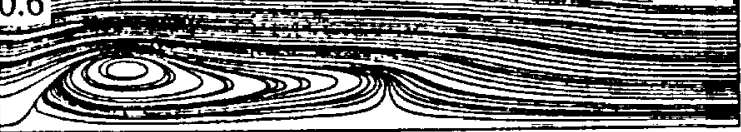

$\Longrightarrow \quad-3=5$ 303 2000 $\Rightarrow \quad t=8$ $\Rightarrow \quad 03$

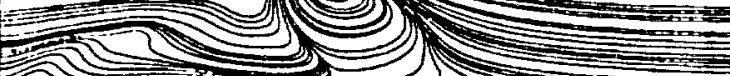

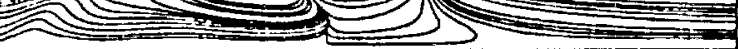
t $t=10$

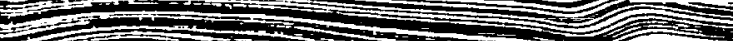

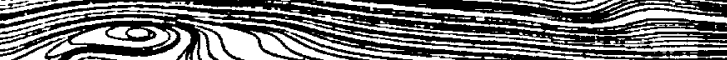
3020 $125 \mathrm{t}=12$

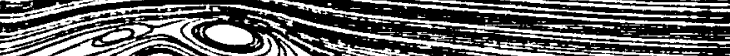

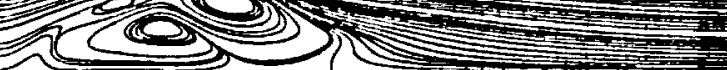
(c)

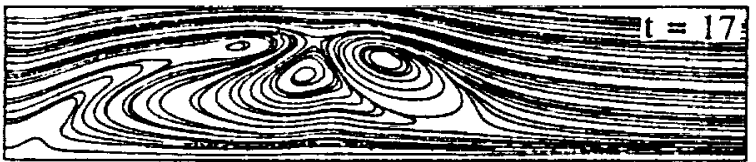

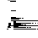

$-$

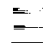

工

$=$

$-$

$-$

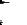

$-$

-

$\overline{5}$

$=$

$-$

$+$
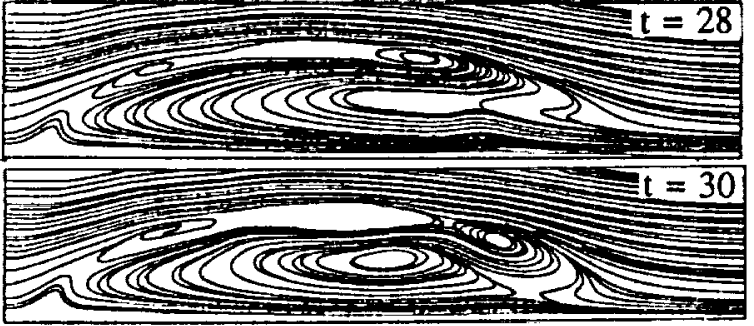
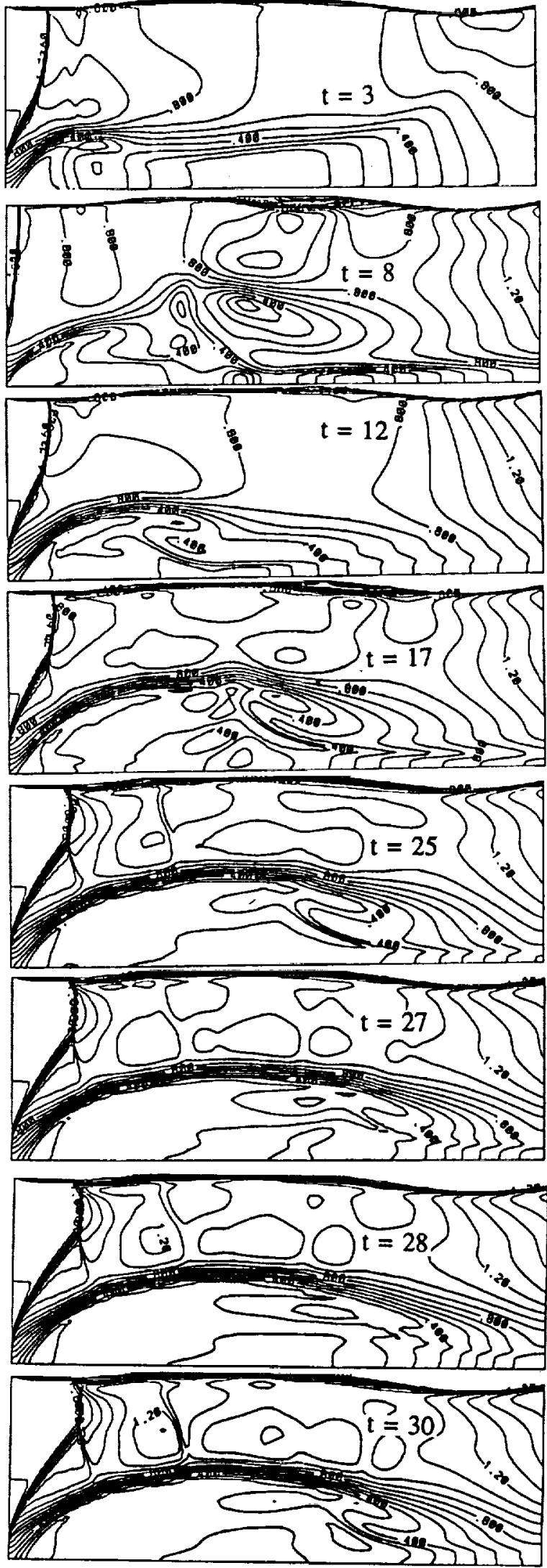

Figure 13. Streamlines and Mach contours for a swirling flow with unsteady multi-bubble breakdowns, $\mathrm{M}_{\infty}=1.75, \beta=0.38, \mathrm{R}_{\mathrm{e}}=100,000$. 



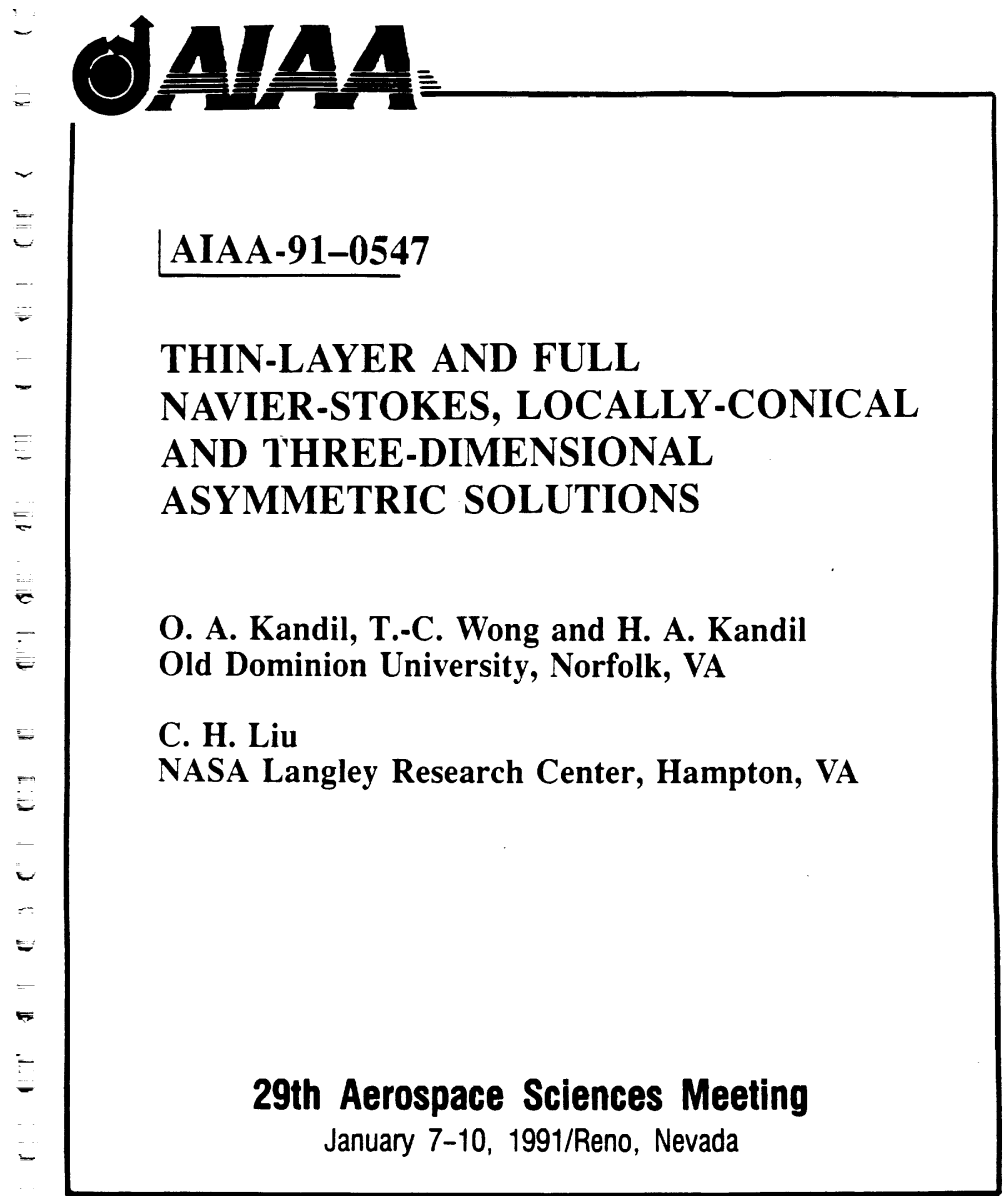

三 For permission to copy or republish, contact the American Institute of Aeronautics and Astronautics 370 L'Entant Promenade, S.W., Washington, D.C. 20024 

THIN-LAYER AND FULL NAVIER-STOKES, LOCALLY-CONICAL AND THREE-DIMENSIONAL ASYMMETRIC SOLUTIONS

\author{
O. A. Kandil*, T.-C. Wong** and H. A. Kandil*** \\ Old Dominion University, Norfolk, VA \\ C. H. Liu $\dagger$ \\ NASA Langley Research Center, Hampton, VA
}

\begin{abstract}
The unsteady, compressible thin-layer and full Navier-Stokes equations are used to numerically simulate steady and unsteady asymmetric, supersonic, locallyconical flows around a $5^{\circ}$-semiapex angle circular cone. The main computational scheme used for the present computations is the implicit, upwind, flux-difference splitting, finite-volume scheme. Comparisons of the solutions using the two sets of equations are presented for the flow asymmetry and its control. Computational studies are also presented to investigate the effects of the freestream Reynolds number and the locally-scaled Reynolds number on the flow asymmetry. These studies are carried out using the full Navier-Stokes equations. Three-dimensional, asymmetric flow solutions are also presented for a $5^{\circ}$. semiapex angle cone of unit length and a cone-cylinder configuration. The three-dimensional solutions are obtained by using the thin-layer equations and short-duration transient side-slip disturbances along with a very fine grid.
\end{abstract}

\section{Introduction}

Highly swept, round and sharp-leading-edge wings and pointed slender bodies are common aerodynamic components to fighter aircraft and missiles. The study of vortex-dominated flow around these isolated aerodynamic components adds to our basic understanding of vortical flows under various conditions including unsteady vortex-dominated flows, vortex-shock interaction, asymmetric vortex flow and vortex breakdown. In this paper, we focus on the problem of asymmetric vortex flow about slender bodies in the high AOA range. The problem is of vital importance to the dynamic stability and controllability of missiles and fighter aircraft.

The onset of flow asymmetry occurs when the relative incidence (ratio of angle of attack to nose semiapex angle) of pointed forebodies exceeds certain critical values. At these critical values of relative incidence, flow asymmetry develops due to natural and/or forced disturbances. The origin of natural disturbances may be a transient side slip. an acoustic disturbance, or likewise disturbance of short duration. The origin of forced disturbances is geometric

\footnotetext{
-Profeasor and Emineat Scholar, Depe of Mecherical Encinearing and Mecherics, Assocince Fellow, AMA.

-Graduate Research Aesiruns, Same Depe, Member AlAA

"*Graduague Research Ascisuat, Same Dept. Member AlAA

tGroup Leader. Theorecieal Flow Physica Brunch, Senior Mamber AlAA
}

This paper is declared a work of the U.S. Government and is not subject to copyright protection in the United States. perturbations due to imperfections in the nose geometric symmetry or likewise disturbances of permanent nature. In addition to the relative incidence as one of the determinable parameters for the onset of flow asymmetry, the freestream Mach number, Reynolds number and shape of the body-cross sectional area are important determinable parameters.

Kandil, Wong and $\mathrm{Liu}^{1}$ used the unsteady, thin-layer Navier-Stokes equations along with two different implicit schemes to simulate asymmetric vortex flows around cones with different cross-section shapes. The numerical investigation was focused on a $5^{\circ}$-semiapex angle circular cone and local conical flow was assumed. The first computational scheme was an implicit, upwind, fluxdifference splitting, finite-volume scheme and the second one was an implicit, central-difference, finite-volume scheme. Keeping the Mach number and Reynolds number constants at 1.8 and $10^{5}$, respectively, the angle of attack was varied from $10^{\circ}$ to $30^{\circ}$. At $\alpha=10^{\circ}$, a steady symmetric solution was obtained and the results of the two schemes were in excellent agreement. At $\alpha=20^{\circ}$ and irrespective of the type or level of the disturbance, a steady asymmetric solution was obtained and the results of the two schemes were in excellent agreement. Two types of flow disturbances were used; a random roundoff error or a random truncation-error disturbance and a controlled transient side-slip disturbance with short duration. For the controlled transient side-slip disturbance, the solution was unique, and for the uncontrolled random disturbance, the solution was also unique with the exception of having the same asymmetry changing sides on the cone. At $\alpha=30^{\circ}$, an unsteady asymmetric solution with vortex shedding was obtained, and the vortex shedding was perfectly periodic. Next, the angle of attack was kept fixed at $20^{\circ}$ and the Mach number was increased from 1.8 to 3.0 with a step of 0.4 . The solutions showed that the asymmetry become weaker as the Mach number is increased. The flow recovered its symmetry when the Mach number reached 3.0. Selected solutions of steady and unsteady asymmetric flows have also been presented for cones with elliptic and diamond cross-sectional areas. Passive control of the flow asymmetry has been tentatively demonstrated by using a fin on the leeward side of the body along the plane of geometric symmetry.

Siclari ${ }^{2}$ used the unsteady, Navier-Stokes equations with a multi-grid, central-difference, finite-volume scheme to solve for steady asymmetric conical flows 
around cones with elliptic, diamond and biparabolic sections. He addressed steady-flow problems similar to those of the present authors in reference 1 . He considered the flow around circular cones with semiapex angles of $5^{\circ}$, $6^{\circ}, 7^{\circ}$ and $8^{\circ}$ at an angle of attack of $20^{\circ}$ and a Reynolds number of $10^{5}$. Varying the Mach number from 1.4 to 3.0 with a step of 0.4 , he showed that the flow recovered its symmetry as the Mach number increased. The higher the semiapex angle was, the lower the Mach number

for the flow to recover it symmetry. Fixing the Mach number at 1.8 , the angle of attack at $20^{\circ}$, the Reynolds number at $10^{5}$ and the semiapex angle at $5^{\circ}$, he decreased the cross-section fineness ratio (ratio of width to length) for different cross-sectional shapes. He showed that the flow recovered its symmetry at a fineness ratio of 0.4 for the elliptic-section cone, at 0.6 for the biparabolic-section cone and at 0.6 for the diamond-section cone.

In a very recent paper by Kandil, Wong, and $\mathrm{Liu}^{3}$. several issues related to the asymmetric flow solutions have been addressed. It has been shown that a unique asymmetric flow solution is obtained irrespective of the size of the minimum grid spacing at the solid boundary. The asymmetry could reverse sides due to the random nature of the disturbance. It has been also shown that for the same flow conditions and same section fineness ratio, diamond-section cones with sharp edges have less flow asymmetry than those of the elliptic-section cones. Moreover, it has been shown that passive control of flow asymmetry of diamond-section cones requires fin heights that are not necessarily equal to the local section width. On the other hand, passive control of flow asymmetry of circular and elliptic-section cones requires fins with heights that are, at least, equal to the local section width. Again, it was also shown that unsteady periodic asymmetric flow with vortex shedding has been predicted.

In reference 4 by Kandil, Wong and Liu, several unsteady, asymmetric vortex flows with periodic vortex shedding for circular and noncircular section cones using the thin-layer Navier-Stokes equations were presented and studied. In reference 5 by Kandil, et. al, the authors addressed the problem of asymmetric flow control using side strakes and the thin-layer Navier-Stokes equations. Two asymmetric flow cases have also been solved using the full Navier-Stokes equations.

In the present paper, we address several issues related to the flow asymmetry around circular cones. Under the locally-conical flow assumption, steady and unsteady asymmetric solutions using the thin-layer and full NavierStokes equations are presented and compared. Also, control of flow asymmetry using vertical fins and side strakes are covered. The solutions for the control cases are carried out using the thin-layer and full Navier-Stokes equations and their results are compared and discussed. Next, the effect of the freestream Reynolds number on the flow asymmetry is studied under the locally-conical flow assumption using the full Navier-Stokes equations. This is achieved by keeping the axial station, at which the locally-conical solution is obtained, constant at the value of unity and changing the Reynolds number. The effect of scaled Reynolds number (Reynolds number based on the local axial station) on the flow asymmetry is also studied using the full Navier-Stokes equations. This is achieved by reducing the axial station and accordingly scaling the Reynolds number, the cross-section diameter, the grid fineness and the computational domain. Finally, three-dimensional flow asymmetry is investigated around a circular cone and a cone-cylinder configuration using the thin-layer Navier-Stokes equations. The purpose of this study is obtain flow asymmetry due to short-duration disturbances and to investigate the effect of Reynolds number, angle of attack and the cylinder afterbody on the flow asymmetry.

\section{Formulation}

\section{Full and Thin-Layer Navier-Stokes Equations}

The conservative form of the dimensionless, unsteady, compressible, full Navier-Stokes equations in terms of time-independent, body-conformed coordinates $\xi^{1}, \xi^{2}$ and $\xi^{3}$ is given by

$$
\frac{\partial \overline{\mathrm{Q}}}{\partial \mathrm{t}}+\frac{\partial \overline{\mathrm{E}}_{\mathrm{m}}}{\partial \xi^{\mathrm{m}}}-\frac{\partial\left(\overline{\mathrm{E}}_{\mathrm{v}}\right)_{\mathrm{s}}}{\partial \xi^{\mathbf{s}}}=0 ; \mathrm{m}=1-3, \mathrm{~s}=1-3
$$

where

$$
\begin{gathered}
\xi^{\mathrm{m}}=\xi^{\mathrm{m}}\left(\mathrm{x}_{1}, \mathrm{x}_{2}, \mathrm{x}_{3}\right) \\
\overline{\mathrm{Q}}=\frac{\hat{\mathrm{q}}}{\mathrm{J}}=\frac{1}{\mathrm{~J}}\left[\rho, \rho \mathrm{u}_{1}, \rho \mathrm{u}_{2}, \rho \mathrm{u}_{3}, \rho \mathrm{e}\right]^{\mathrm{t}}
\end{gathered}
$$

$$
\begin{aligned}
\bar{E}_{m} & \equiv \text { inviscid flux } \\
& =\frac{1}{J}\left[\partial_{k} \xi^{m} E_{k}\right]^{l} \\
& =\frac{1}{J}\left[\rho U_{m}, \rho u_{1} U_{m}+\partial_{1} \xi^{m} p, \rho u_{2} U_{m}\right. \\
& \left.+\partial_{2} \xi^{m} p, \rho u_{3} U_{m}+\partial_{3} \xi^{m} p,(\rho e+p) U_{m}\right]^{\mathrm{t}}
\end{aligned}
$$

$$
\begin{aligned}
& \left(\bar{E}_{\mathrm{v}}\right)_{\mathrm{s}} \equiv \text { viscous and heat-conduction flux in } \xi^{s} \\
& \begin{array}{l}
\text { direction } \\
=\frac{1}{\mathrm{j}}\left[0, \partial_{\mathrm{k}} \xi^{3} n_{\mathrm{k} 1}, \partial_{\mathrm{k}} \xi^{3} n_{\mathrm{k} 2}, \partial_{\mathrm{k}} \xi^{3} n_{\mathrm{k} 3}\right. \text {, }
\end{array} \\
& \left.\partial_{k} \xi^{3}\left(u_{n} \eta_{k n}-q_{k}\right)\right]^{t} ; \quad k=1-3, n=1-3 \\
& \mathrm{U}_{\mathrm{m}}=\partial_{\mathrm{k}} \xi^{\mathrm{m}} \mathrm{u}_{\mathrm{k}}
\end{aligned}
$$

The first element of the three momentum elements of Eq. (5) is given by

$$
\begin{aligned}
\partial_{\mathrm{k}} \xi^{s} \nu_{\mathrm{k}} & \equiv \frac{\mathrm{M}_{\propto \mu} \mu}{\operatorname{Re}}\left[\left(\partial_{\mathrm{k}} \xi^{s} \partial_{1} \xi^{\mathrm{n}}-\frac{2}{3} \partial_{1} \xi^{s} \partial_{\mathrm{k}} \xi^{\mathrm{n}}\right) \frac{\partial \mathrm{u}_{\mathrm{k}}}{\partial \xi^{\mathrm{n}}}\right. \\
& \left.+\partial_{\mathrm{k}} \xi^{s} \partial_{\mathrm{k}} \xi^{\mathrm{n}} \frac{\partial \mathrm{u}_{1}}{\partial \xi^{s}}\right]
\end{aligned}
$$


The second and third elements of the momentum elements are obtained by replacing the subscript 1 , everywhere in Eq. (7), with 2 and 3 , respectively. The last element of Eq. (5) is given by

$$
\begin{aligned}
\partial_{k} \xi^{s}\left(u_{p} \pi_{k p}\right. & \left.-q_{k}\right) \equiv \frac{M_{x} \mu}{\operatorname{Re}}\left[\left(\partial_{k} \xi^{s} \partial_{p} \xi^{n}\right.\right. \\
& \left.-\frac{2}{3} \partial_{p} \xi^{s} \partial_{k} \xi^{n}\right) u_{p} \frac{\partial u_{k}}{\partial \xi^{n}} \\
& +\partial_{k} \xi^{s} \partial_{k} \xi^{n} u_{p} \frac{\partial u_{p}}{\partial \xi^{n}} \\
& \left.+\frac{1}{(\gamma-1) P_{r}} \partial_{k} \xi^{s} \frac{\partial\left(a^{2}\right)}{\partial \xi^{n}}\right] ; p=1-3
\end{aligned}
$$

The single thin-layer approximations of the full NavierStokes equations demand that we only keep the derivatives in the normal direction to the body, $\xi^{2}$, in the viscous and heat flux terms in Eqs. (1), (7) and (8). Thus, we let $s=2$ for the term $\frac{\partial\left(\hat{C}_{v}\right)}{\partial \xi_{1}}$ in Eq. (1) and $s=2$ and $n=2$ in Eqs. (7) and (8). These equations reduce to

$$
\begin{aligned}
& \frac{\partial \overline{\mathrm{Q}}}{\partial \mathrm{t}}+\frac{\partial \overline{\mathrm{E}}_{\mathrm{m}}}{\partial \xi^{\mathrm{m}}}-\frac{\partial\left(\overline{\mathrm{E}}_{\mathrm{v}}\right)_{2}}{\partial \xi^{2}}=0 \\
& \partial_{k} \xi^{2} \eta_{k 1} \equiv \frac{M_{x} \mu}{\operatorname{Re}}\left(\psi \partial_{1} \xi^{2}+\phi \frac{\partial u_{1}}{\partial \xi^{2}}\right) \\
& \partial_{k} \xi^{2}\left(u_{p} r_{k p}-q_{k}\right) \equiv \frac{M_{\propto} \mu}{R e}\{\psi W \\
& +\phi\left[\frac{1}{2} \frac{\partial}{\partial \xi^{2}}\left(u_{1}^{2}+u_{2}^{2}+u_{3}^{2}\right)\right. \\
& \left.\left.+\frac{1}{(\gamma-1) P_{r}} \frac{\partial\left(\mathrm{a}^{2}\right)}{\partial \xi^{2}}\right]\right\}
\end{aligned}
$$

where

$$
\phi_{1}=\partial_{k} \xi^{2} \partial_{k} \xi^{2}, \psi=\frac{1}{3} \partial_{k} \xi^{2} \frac{\partial u_{k}}{\partial \xi^{2}}, W=\partial_{p} \xi^{2} u_{p}
$$

The reference parameters for the dimensionless form of the equations are $L, a_{\infty}, L / a_{\infty}, \rho_{\infty}$ and $\mu_{\infty}$ for the length, velocity, time, density and molecular viscosity, respectively. The Reynolds number is defined as $\mathbf{R e}=$ $\rho_{\infty} V_{\infty} \mathrm{L} / \mu_{\infty}$, where $\mathrm{L}$ is the body length, and the pressure, $p$, is related to the total energy per unit mass and density by the gas equation

$$
\mathrm{p}=(\gamma-1) \rho\left[\mathrm{e}-\frac{1}{2}\left(\mathrm{u}_{1}^{2}+\mathrm{u}_{2}^{2}+\mathrm{u}_{3}^{2}\right)\right]
$$

The viscosity is calculated from the Sutherland law

$$
\mu=\mathrm{T}^{3 / 2}\left(\frac{1+\mathrm{C}}{\mathrm{T}+\mathrm{C}}\right), \mathrm{C}=0.4317
$$

and the Prandtl number $P_{r}=0.72$. In Eqs. $(1)-(12)$, the indicial notation is used for convenience.
Boundary and Initial Conditions

Boundary conditions are explicitly implemented. They include inflow-outflow conditions and solidboundary conditions. At the plane of geometric symme. try, periodic conditions are used for symmetric or asymmetric flow applications on the whole computational domain (right and left domains). At the farfield inflow boundaries freestream conditions are specified since we are dealing with supersonic flows, while at the far-field outflow boundaries first-order extrapolation from the interior points is used. On the solid boundary, the no-slip and no-penetration conditions are enforced; $u_{1}=u_{2}=u_{3}$ $=0$ and the normal pressure gradient is set equal to zero. For the temperature, the adiabatic boundary condition is enforced on the solid boundary.

For the passive control applications using vertical fins, double thin-layer Navier-Stokes equations are used, where one thin-layer is used normal to the body and another thin-layer is used normal to the fin surface. For these applications, solid-boundary conditions are enforced on both sides of the fin.

The initial conditions correspond to the uniform flow with $u_{1}=u_{2}=u_{3}=0$ on the solid boundary.

\section{Computational Scheme}

The main computational scheme used to solve the thin-layer and full Navier-Stokes equations is an implicit, upwind, flux-difference splitting, finite-volume scheme. It employs the flux-difference splituing scheme of Roe. The Jacobian matrices of the inviscid fluxes, $A_{s}=\frac{\partial E_{i}}{\partial q}$; $s=1-3$, are split into backward and forward fluxes according to the signs of the eigenvalues of the inviscid Jacobian matrices. Flux limiters are used to eliminate oscillations in the shock region. The viscous and heat-flux terms are centrally differenced. The resulting difference equation is solved using approximate factorization in the $\xi^{1}, \xi^{2}$ and $\xi^{3}$ directions. The resulting computer program can be used to solve the thin-layer Navier-Stokes equations and the full Navier-Stokes equations. This code is a modified version of the CFL3D which is currently called "FTNS3D". In this code, the implicit, flux-vector splitting, finite-volume scheme, which is based on the Van-Leer scheme ${ }^{6}$, can also be used instead of the fluxdifference splitting scheme.

\section{Computational Applications and Discussions}

\section{Locally-Conical Asymmetric Flow Applications}

Locally-conical solutions of the thin-layer or full Navier-Stokes equations are obtained using one of two methods. In the first method, the governing equations 
are transformed using the conical-coordinate transformation. Invoking the conical flow conditions which require that the flow variables be independent of the radial distance (or axial distance, depending on the transformation) from the cone vertex, equating the radial distance (or axial distance) which appears in the transformed equations to a constant (equals to unity in most of the present locally-conical solutions), the resulting equations are solved on one spherical (or cross-flow) surface. In the second method, the three-dimensional flow equations are solved on two spherical (or cross-flow) surfaces which are located in the very near proximity of a constant radial (or axial) distance. During the psuedo-time or accuratelime stepping, the flowfield vector is forced to be equal at the corresponding grid centers on the two surfaces. This method is used in the present paper to obtain locallyconical solutions. The resulting solutions from these two methods are the same locally-conical solutions. These solutions correspond to the specified radial (or axial) distance and hence they change as we change the radial (or axial) distance. The reason behind that is simply because the ransformed equations, according to the first method, are not self-similar and hence they are not globally conical. This is shown below by developing the transformed equations of the first method. Considering the unsteady, compressible, Navier-Stokes equations in the Cartesian coordinates,

$$
\frac{\partial q}{\partial t}+\frac{\partial\left(E-E_{v}\right)_{i}}{\partial x_{i}}=0 \quad ; i=1-3
$$

introducing the conical coordinates,

$$
\eta_{1}=\frac{x_{1}}{x_{3}}, \quad \eta_{2}=\frac{x_{2}}{x_{3}}, \quad \eta_{3}^{2}=x_{i} x_{i}
$$

and using the chain rule, Eq. (15) is transformed to

$$
\begin{gathered}
\frac{\eta_{3}}{\mathrm{~m}} \frac{\partial \mathrm{q}}{\partial \mathrm{t}}+\frac{\partial}{\partial \eta_{1}}\left(\overline{\mathrm{E}}-\overline{\mathrm{E}}_{\mathrm{v}}\right)_{1}+\frac{\partial}{\partial \eta_{2}}\left(\overline{\mathrm{E}}-\overline{\mathrm{E}}_{\mathrm{v}}\right)_{2} \\
+\frac{\eta_{3}}{m^{2}} \frac{\partial}{\partial \eta_{3}}\left(\bar{E}-\bar{E}_{v}\right)_{3}+2\left(\bar{I}-\bar{I}_{\mathrm{v}}\right)=0
\end{gathered}
$$

where

$$
\begin{aligned}
& \mathrm{m}=\sqrt{1+\eta_{1}^{2}+\eta_{2}^{2}}, \quad \overline{\mathrm{E}}_{1}=\mathrm{E}_{1}-\eta_{1} \mathrm{E}_{3}, \overline{\mathrm{E}}_{2} \\
& \quad=\mathrm{E}-\eta_{2} \mathrm{E}_{3}, \overline{\mathrm{E}}_{3}=\mathrm{E}_{3}+\eta_{1} \mathrm{E}_{1}+\eta_{2} \mathrm{E}_{2}, \\
& \overrightarrow{\mathrm{I}}=\overline{\mathrm{E}}_{3}, \overline{\mathrm{E}}_{\mathrm{v} 1}=\mathrm{E}_{\mathrm{v} 1}-\eta_{1} \mathrm{E}_{\mathrm{v} 3}, \overline{\mathrm{E}}_{\mathrm{v} 2} \\
& =\mathrm{E}_{\mathrm{v}}-\eta_{2} \mathrm{E}_{\mathrm{v} 3}, \overline{\mathrm{E}}_{\mathrm{v} 3}=\mathrm{E}_{\mathrm{v} 3}+\eta_{1} \mathrm{E}_{\mathrm{v} 1}+\eta_{2} \mathrm{E}_{\mathrm{v} 2}, \\
& \overline{\mathrm{I}}_{\mathrm{v}}=\overline{\mathrm{E}}_{\mathrm{v} 3}
\end{aligned}
$$

The conical flow condition requires the flow variables be independent of the coordinate $\eta_{3}$ (radial distance). Invoking this condition in $\mathrm{Eq}$. (17) by dropping the derivatives with respect to $\eta_{3}, \mathrm{Eq}$. (17) reduces to

$$
\frac{\eta_{3}}{\mathrm{~m}} \frac{\partial \mathrm{q}}{\partial \mathrm{t}}+\frac{\partial}{\partial \eta_{1}}\left(\overline{\mathrm{E}}-\overline{\mathrm{E}}_{\mathrm{v}}\right)_{1}+\frac{\partial}{\partial \eta_{2}}\left(\overline{\mathrm{E}}-\overline{\mathrm{E}}_{\mathrm{v}}\right)_{2}+2\left(\overline{\mathrm{I}}-\overline{\mathrm{I}}_{\mathrm{v}}\right)=0
$$

It is obvious that the unsteady term includes $\pi_{3}$. Moreover, the viscous terms $\frac{\partial \bar{U}_{z 1}}{\partial \eta_{1}}, \frac{\partial \bar{F}_{r_{2}}}{\partial \eta_{2}}$ and $\overline{\mathrm{I}}_{v}$ include $\eta_{3}$, and hence Eq. (19) is not self-similar. The explicit dependence of the viscous terms on $\eta_{3}$ can be shown through one of the elements of these vectors. For example, we consider

$$
\begin{aligned}
& \frac{\partial}{\partial \eta_{1}}\left(\tau_{\mathbf{x x}}-\eta_{1} \tau_{\mathbf{x z}}\right)=\frac{\mathrm{M}_{x}}{\mathrm{R}_{3}} \frac{\partial}{\partial \eta_{1}}\left[\mu \left(2 \frac{\partial \mathrm{u}_{1}}{\partial \mathrm{x}}\right.\right. \\
& \left.\left.-\frac{2}{3}\left(\frac{\partial \mathrm{u}_{1}}{\partial \mathrm{x}}+\frac{\partial \mathrm{u}_{2}}{\partial \mathrm{y}}+\frac{\partial \mathrm{u}_{3}}{\partial z}\right)-\eta_{1}\left(\frac{\partial \mathrm{u}_{1}}{\partial z}+\frac{\partial \mathrm{u}_{3}}{\partial \mathrm{x}}\right)\right)\right] \\
& =\frac{\mathrm{M}_{\mathrm{x}} \mathrm{m}}{\mathrm{R}_{\mathrm{t}} \eta_{3}} \frac{\partial}{\partial \eta_{1}}\left[\mu \left(\left(\frac{4}{3}-\eta_{1}^{2}\right) \frac{\partial \mathrm{u}_{1}}{\partial \eta_{1}}\right.\right. \\
& \left.\left.+\eta_{1} \frac{\partial \mathrm{u}_{1}}{\partial \eta_{2}}-\frac{2}{3} \frac{\partial \mathrm{u}_{2}}{\partial \eta_{2}}-\frac{\eta_{1}}{3} \frac{\partial \mathrm{u}_{3}}{\partial \eta_{1}}+\frac{2}{3} \eta_{2} \frac{\partial \mathrm{u}_{3}}{\partial \eta_{2}}\right)\right]
\end{aligned}
$$

Thus, the unsteady term and viscous terms are scaled by the radial distance $\eta_{3}$ and $\mathrm{Eq}$. (19) does not represent a globally-conical flow. The best to be done to make use of this equation is to select a constant value for $\eta_{3}$, and solve the resulting equation for what we call "locallyconical flow". If $\eta_{3}$ is assigned another constant value, the resulting equation will have another scale for the unsteady term and viscous terms. It is concluded that Eq. (19) becomes globally conical if the unsteady term and viscous terms vanish, and hence only the steady Euler equations are globally conical.

Next, we present comparison of the solutions using the thin-layer and full Navier-Stokes equations. We also present steady flow solutions using different Reynolds numbers keeping $\eta_{3}=1$ and steady solutions using $\eta_{3}$ $=0.5,0.25,0.1$ and $R_{e}=50,000 ; 25,000 ; 10,000$; respectively.

Steady Asymmetric Flow $\left(\alpha=20^{\circ}, \mathrm{M}_{x}=1.8, \mathbf{R}_{\mathrm{e}}=10^{5}\right)$

Supersonic flow around a $5^{\circ}$-semiapex angle circular cone at $20^{\circ}$ angle of attack, freestream Mach number of 1.8 and Reynolds number of $10^{5}$ is considered. The thin-layer and full Navier-Stokes equations are used to solve for asymmerric flow on a grid of $161 \times 81$ points in the circumferential and normal directions, respectively. The grid is generated by using a modified Joukowski transformation with a geometric series for grid clustering in the normal direction. The minimum grid spacing in the nomal direction at the solid boundary is $10^{-4}$. The maximum radius of the computational domain is $21 \mathrm{r}$, where $r$ is the radius of the circular cone at the axial station of unity. Figure 1 shows the results of thinlayer solution. The color graphics figure shows six snap shots for the evolution of the steady asymmetric solution during 10,000 iteration steps. The number from $1-6$ on the total-pressure-loss contours correspond to the number from 1-6 on the logarithmic residual-iterations curve. It is seen that the solution is symmetric during the first 3000 iteration when the residual drops to machine zero (No. 1). Then, the solution becomes slightly asymmetric as the residual grows up (Nos. 2, 3). As the residual drops again to machine zero, the flow asymmetry becomes strong until it reaches to the stable asymmetric solution 
(Nos. 4, 5, 6). The final total-pressure-loss contour and the corresponding surface-pressure coefficient are also shown.

The full Navier-Stokes solution is shown in Fig. 2. The residual-error drops 4.5 orders of magnitude in 2,000 steps, increases one order of magnitude after a total of 3,000 steps and then drops to machine zero in a total number of iteration steps of 6,000 . It is clear that the full Navier-Stokes equations produce the asymmetric solution faster than the thin-layer Navier-Stokes equations. The total-pressure-loss contours show that the full NavierStokes solution produces thicker shear layers than those of the thin-layer solution. More contour resolution in the vortex cores is produced by the full Navier-Stokes solutions than that of the thin-layer solution. Finally, the free-shear layer on the body right-side of the full NavierStokes solution is shorter than that of the thin-layer solution. However, the $C_{p}$ figures of the two solutions are exactly the same.

\section{Asymmetric Flow Control Using Vertical Fins $(h=0.5 r, h=r)$}

Figures 3 and 4 show comparisons of the thin-layer and full Navier-Stokes solutions for the control of the flow of the preceding case. Two vertical fins of heights $h$ $=0.5 \mathrm{r}$ and $\mathrm{r}$ are placed in the leeward plane of geometric symmetry, where $r$ is the cone local radius. Figures 3 and 4 show the total-pressure-loss contours and the surfacepressure coefficient. The thin-layer solutions are shown on the left and the full Navier-Stokes solutions are shown on the right. With $h=0.5 r$ and using the thin-layer equations, two vortex cores which are connected to each other and to the body through free-shear layers, develop from the left side of the body. From the right side of the body, a free-shear layer develops and crosses over the fin to the left side of the body. It produces two vortex cores; one at each comer of the body-fin juncture with secondary separation below them. This case has been solved accurately in time but it does not show any vortex shedding or unsteadiness. When the fin height is increased to $h=r$, perfect flow symmetry is obtained.

With $h=0.5 r$ and using the full Navier-Stokes equations, a mirror image of the vortex system and flow separations of the thin-layer solutions is obtained. This is due to the random nature of the disturbance. However, the freeshear layer and vortex cores on the right are thicker and shorter in height than those of the thin-layer solution. However, the $C_{p}$ coefficients of the two sets of equations are exact mirror image of each other. With $h=r$, the full Navier-Stokes equations produce the exact same solution as that of the thin-layer equations. The reason behind the flow asymmetry with $h=0.5 r$ is that the free-shear layer from the right-hand side of the body is still higher than the fin height, which allows the flow disturbances from the right and left side to interact.
Asymmetric Flow Control Using Side

Strakes $(h=0.3 r)$

Figure 5 shows a comparison of the thin-layer and full Navier-Stokes solutions for the control of the flow of the case of Fig. 1. Side strakes of height $h=0.3 r$. when $r$ is the local radius of the cone, are used for this purpose. The grid used is generated using a hyperbolic grid generator with transfinite grid interpolation to refine the grid at the strake sharp edge. While the thin-layer solution shows perfect symmetric fow, the full NavierStokes solution shows slightly asymmetric flow. This can be seen by comparing both the total-pressure-loss contours and the surface-pressure coefficient of the two solutions. Both solutions are obtained using a grid of $161 \times 81$ with the same transfinite interpolation. Both surface-pressure coefficients show a jump in the pressure value at the leading edges of the strakes. It should be noted that side-strakes produce higher lift than that of the vertical fin control.

Unsteady Asymmetric Flow ( $\alpha=30^{\circ}, \mathrm{M}_{\infty}$ $=1.8, R_{e}=10^{5}$ )

Figures 6 and 7 show comparisons of the full NavierStokes solutions and the thin-layer solutions for unsteady asymmetric flow with vortex shedding. The solutions are obtained by using the full Navier-Stokes equations with a grid of $161 \times 81$ and the flux difference splitting (FDS) scheme, thin-layer equations with a grid of $161 \times 81$ and the FDS scheme, thin-layer equations with a grid of $161 \times 81$ and the flux-vector spliting (FVS) scheme, and full Navier-Stokes equations with a grid of $241 \times 81$ and the FDS scheme. Typical of all the four solutions, the residual error curves show a drop in the error followed by a transient response and ending by a periodic response. The lift coefficient curves show the final periodic response. The first three solutions exactly show the same number of time steps $\left(\Delta t=10^{-3}\right)$ of 700 for one-half of the cycle of vortex shedding. The full Navier-Stokes solution on the fine grid (Fig. 7) shows a slightly bigger number of time steps $\left(\Delta t=10^{-3}\right)$ of 725 for one-half of the cycle of vortex shedding. This is expected of the full Navier-Stokes equations on the fine grid since the flow has more real damping and viscous-force resolution than the other three solutions. In the fourth case, the damping will elongate the period of shedding and hence it reduces the frequency of shedding. The frequency of shedding of the first three solutions is 4.488 and the frequency of shedding of the fourth solution is 4.333 .

\section{Asymmetric Flow Control Using a Vertical Fin $(h=r)$}

Figure 8 shows the full Navier-Stokes solution of asymmetric flow control using a vertical fin of $h=r$. This is the flow considered in the preceding case. It is seen that the vertical fin height is not enough to eliminate the flow asymmetry. The flow is still asymmetric but steady. 
Effect of Decreasing the Reynolds Number Keeping the Axial Distance Fixed $\left(x=1, \alpha=20^{\circ}, M_{x}\right.$ $=1.8, R_{e}=20,000 ; 15,000 ; 10,000$ )

Figure 9 shows the results of a study to investigate the effect of reducing the Reynolds number on the flow asymmetry. The full Navier-Stokes equations are used on a grid of $241 \times 81$. As the Reynolds number is decreased keeping $x=1$, the flow asymmetry decreases but it does not completely diminish. In this study, the computational domain is kept fixed in size and the minimum grid spacing at the solid boundary is kept at $10^{-4}$. It should be stressed here that with decreasing the Reynolds number to the values considered, only the full Navier-Stokes solutions on a relatively fine grid will be valid. Strictly speaking, the cut-off Reynolds number for flow asymmetry is below 10,000 .

Effect of Decreasing the Reynolds Number and the Axial Distance $\left(x=0.5,0.25,0.1, \alpha=20^{\circ}\right.$, $M_{x}=1.8, R_{e}=50,000 ; 25,000 ; 10,000$ )

Figure 10 shows the results of a study to investigate the effect of reducing the axial distance and proportionally the Reynolds number, the computational domain and the minimum grid spacing at the solid boundary. In other words, we are investigating whether the flow is selfsimilar or not. The solutions shown in the figure ensure that the flow is not self similar as the problem is scaled at each axial station. However, in another numerical study, we have shown that if the Reynolds number is increased by the same ratio as that of decreasing the axial station (e.g. if $x=0.5$ then $R_{e}=200,000$ such that $x R_{e}=100,000$ ) and the problem is solved for the corresponding reduced radius of the cone section and its reduced computational domain, the asymmetric flow solution is self similar at any section.

$$
\begin{aligned}
& \text { Unsteady Asymmetric Flow }\left(\alpha=35^{\circ}\right. \text {, } \\
& M_{\infty}=1.8, R_{e}=10^{5}
\end{aligned}
$$

Figure 11 shows snap shots of the unsteady asymmetric flow of the full Navier-Stokes equations for the same circular cone as the angle of attack is increased to $35^{\circ}$. The residual error curve and the lift coefficient curve show the same typical responses as those of Figs. 6 and 7. The period of shedding is smaller and equals to $550 \times 10^{-3}=0.55$ and the corresponding shedding frequency is 11.424 .

\section{Three-Dimensional Flow Applications}

In Figs. 12-19, we present the results of threedimensional asymmetric flow solutions of the thin-layer equations on a grid of $161 \times 81 \times 65$ in the circular, normal and axial directions, respectively. There are several issues to be answered through the present computational study. First, for the same circular cone and for the same flow conditions (angle of atack, Mach number and Reynolds number), will the three-dimensional flow solution be the same as that of the locally-conical solution of Fig. 1? If the answer is negative, the next question to address is: Is there a length scale which relates the three-dimensional solution to the locally-conical solution? The second issue to be addressed is the effect of the Reynolds number, the angle of attack and the cylindrical-afterbody length on the three-dimensional flow asymmetry?

To address these issues, a $5^{\circ}$-semiapex angle circular cone of unit length (cone length is the characteristic length) is considered. The three-dimensional grid of $161 \times 81 \times 65$ is generated by using a modified Joukowski transformation at axial stations. The grid is clustered in the normal direction of the body using a geometric series with the minimum grid spacing of $10^{-5}$ at the vertex and $10^{-6}$ at the axial station of unit length. A typical grid is shown in Fig. 12. With the flow conditions set at $\alpha=$ $20^{\circ}, M_{\infty}=1.8$ and $R_{e}=10^{5}$, which are the same conditions as those of the locally conical flow of Fig. 1, the three-dimensional solution produced a symmetric flow, unlike the local-conical solution which produces asymmetric steady flow. The reason for the difference is well understood since the local-conical solution is obtained at an axial station of $x=1.0$. Hence, a length scale is involved in the Reynolds number, as can be seen from the analytical conical equation for steady viscous flow, Eq. (19). Next, the search is directed at obtaining asymmetric flow solutions for the three-dimensional cone flow. In Fig. 13, we show the solution for the same cone at $\alpha$ $=40^{\circ}, M_{\infty}=1.4$ and $R_{e}=4 \times 10^{6}$. It is seen that the solution is asymmetric and is nearly self-similar over a long axial distance of the cone length.

Next, the Reynolds number is increased to $6 \times 10^{6}$ and $8 \times 10^{6}$ keeping the other flow conditions constant at $a$ $=40^{\circ}$ and $\mathrm{M}_{x}=1.4$. Figures 14 and 15 show strong asymmetric-flow solutions with already shed vortices. It should be noticed that the flow asymmetry changes sides as we move in the downstream direction. Hence, we have spatial asymmetric vortex shedding which is qualitatively similar to the temporal asymmetric vortex shedding of the locally-conical flow solutions of Figs. 6,7 and 11. The flow instability is of spatial type.

In an attempt to address the issue of the effect of the cylindrical afterbody on the flow asymmetry, a cylindrical afterbody of unit length is added to the unit conical forebody. The flow configuration is solved for the flow conditions of the isolated cone of Fig. 13. The results are shown in Fig. 16. Comparing the total-pressureloss contours of Figs. 13 and 16, we see that the flow asymmetry is stronger for the cone-cylinder configuration in comparison with that of the cone alone. It should be noted that inside the shock cone surrounding the conecylinder configuration, subsonic flow exists and hence the downstream boundary has an upstream effect. Figures 17 and 18 show the total-pressure-loss contours and surfacepressure coefficients in cross-flow planes for the cases of Figs. 15 and 16. 
Finally, the angle of attack is increased to $50^{\circ}$ keeping the Mach number and Reynolds number constants at 1.4 and $8 \times 10^{6}$, respectively. The solution is obtained accurately in time. In Fig. 19, we show two snap shots of the solution at the time steps of $n=10,116$ and 11,818 which correspond to $t=0.10116$ and 0.11818 , respectively, since $\Delta t=10^{-5}$. Comparing the two solutions, one can see vortex shedding. This case of three-dimensional unsteady vortex shedding is computationally very expensive since the stable time step is $10^{-5}$, and hence it was not completed.

\section{Concluding Remarks}

In the present paper, the unsteady, compressible thinlayer and full Navier-Stokes equations have been used to solve for steady and unsteady asymmetric flows and their passive control around a $5^{\circ}$-semiapex angle cone. For the steady locally-conical asymmetric solutions, we have shown that the full Navier-Stokes solutions produce thicker and shorter free-shear layers than those of the thin-layer solutions. For the unsteady locally-conical asymmetric solutions, we have shown that the full NavierStokes solutions on a fine grid produce longer periods of vortex shedding and hence smaller shedding frequencies than those of the thin-layer solutions. Next, we addressed the effects of reducing the Reynolds number on the flow asymmetry. First, the Reynolds number is reduced keeping the axial distance constant. We have shown that the cut-off Reynolds number for the flow asymmetry is less than 10,000 . Second, the Reynolds number is reduced along with the proportional reduction of the axial distance, the computational domain and the minimum grid spacing. We have shown that the flow asymmetry disappears below the Reynolds number value of 25,000 . Moreover, we have shown that the flow is not globally conical. Finally, the three-dimensional flow problem is addressed for the same cone and a cone-cylinder configuration. Flow asymmetry has been obtained using short-duration disturbances. The flow asymmetry becomes stronger as the Reynolds number and the angle of attack are increased. It also becomes strong due to the addition of a cylindrical afterbody. Unsteady flow asymmery has also been obtained. It has also been noticed that for certain flow conditions, the flow asymmetry shows spatial vortex shedding which is qualitatively similar to the temporal vortex shedding of the unsteady locally-conical asymmetric flow.

\section{Acknowledgement}

This research work has been supported by the NASA Langley Research Center under grants No. NAS. 1-18584-71 and NAG-1-994. The authors would like to acknowledge the computational resources provided by the NAS-Ames computing facilities and the NASA-Langley computing facilities.

\section{References}

1. Kandil, O. A., Wong, T-C. and Liu, C. H., "Prediction of Steady and Unsteady Asymmetric Vortical Flow Around Cones," AIAA 90-0598, January 1990. Also to appear in AIAA Journal, Vol. 29, No. 7, 1991.

2. Siclari, M. J., "Asymmetric Separated Flows at Supersonic Speeds," AIAA 90-0595, January 1990.

3. Kandil, O. A., Wong, T.C. and Liu, C. H., "Asymmetric Flow Around Cones with Noncircular Sections," AGARD Symposium on Missile Aerodynamics, AGARD CP No. 493, Friedrickshafen, Germany, October 1990, pp. 16.1-16.11.

4. Kandil, O. A. Wong, T-C. and Liu, C. H., "Numerical Simulation of Steady and Unsteady Asymmetric Vortical Flows," ASME Symposium on Non-Steady Fluid Dynamics, FED-Vol. 92, Toronto, Canada, June 1990, pp. 99-108.

5. Kandil, O. A., Wong, T.C., Kandil, H. A. and Liu, C. H., "Computation and Control of Asymmetric Vortex Flow Around Circular Cones Using Navier-Stokes Equations," ICAS Paper No. 3.5.3, Vol. 2, Stockholm, Sweden, September 1990, pp. 883-893.

6. Rumsey, L. C. and Anderson, W. Kyle, "Some Numerical Aspects of Unsteady Navier-Stokes Computations of Unsteady Navier-Stokes Computations over Airfoils Using Dynamic Meshes," AIAA 88-0329, January 1988. 


\section{Steady Asymmetric Flow $\alpha=20^{\circ}, M=1.8, \operatorname{Re}=100,000$ Total-Pressure-Loss Contours}
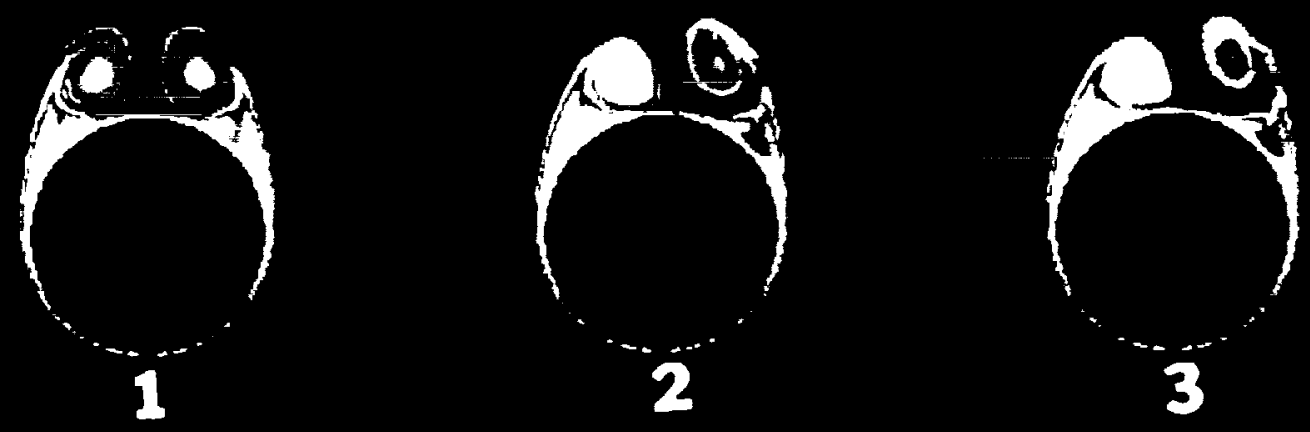

2

3
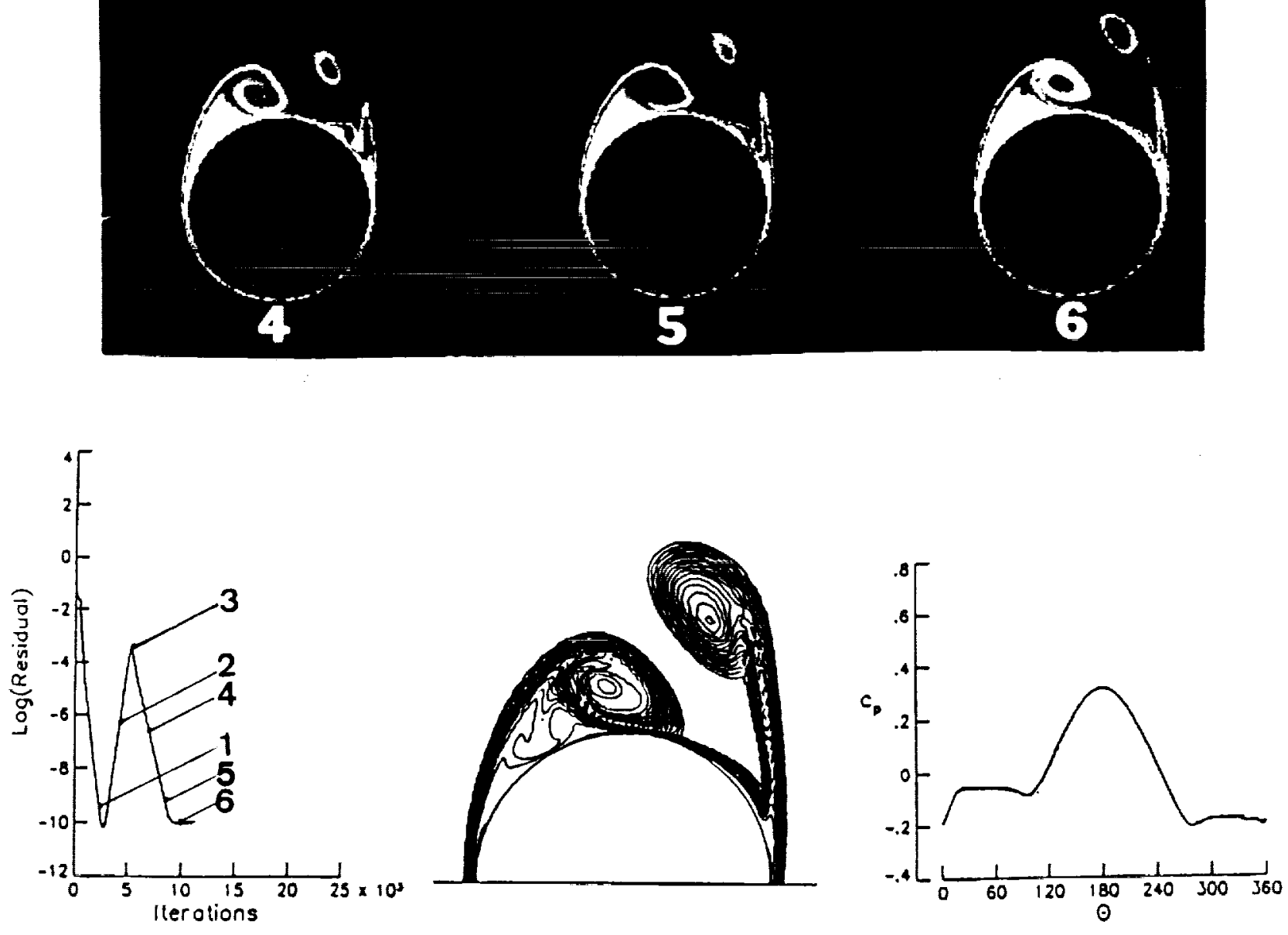

Thin-Layer Navier-Stokes Solution

Figure 1. Evolution of a steady flow asymmerty, total-pressure-loss contours, logarithmic residual history, converged solution, surface-pressure coefficient, thin-layer solution. 

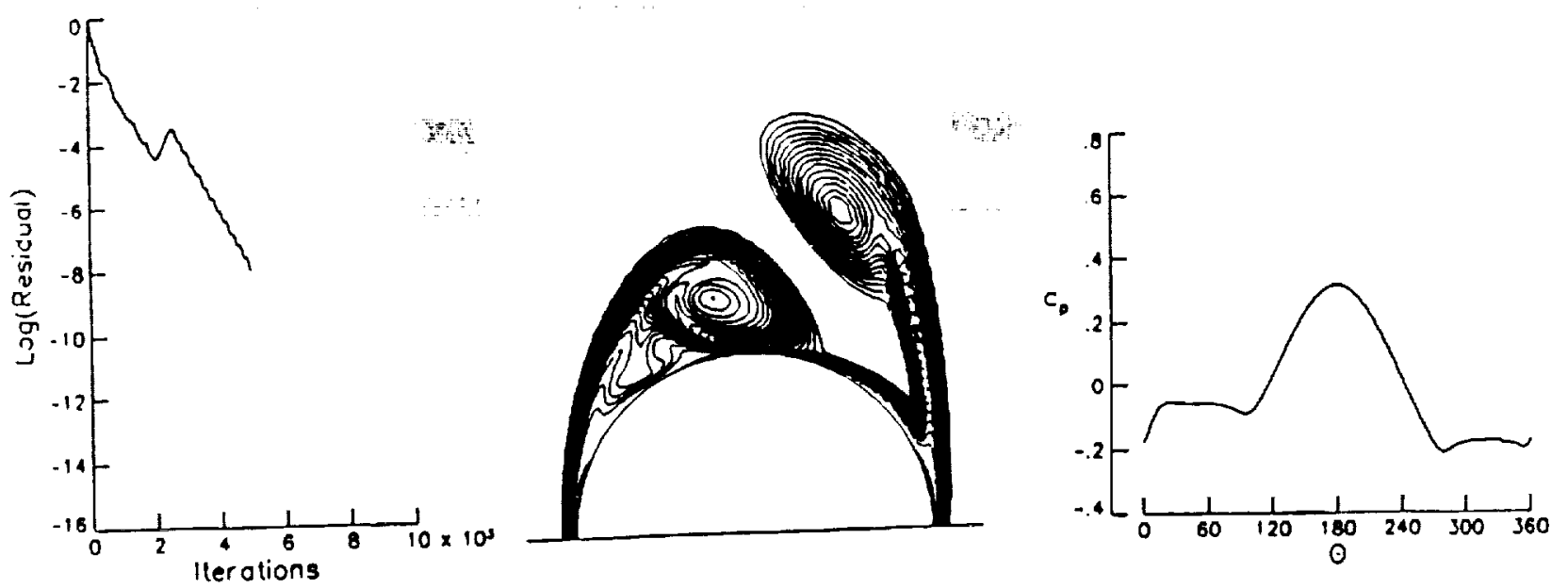

Figure 2. A steady flow asymmetry, logarithmic residual history, converged solution, surface-pressure coefficient, full Navier-Stokes solution.
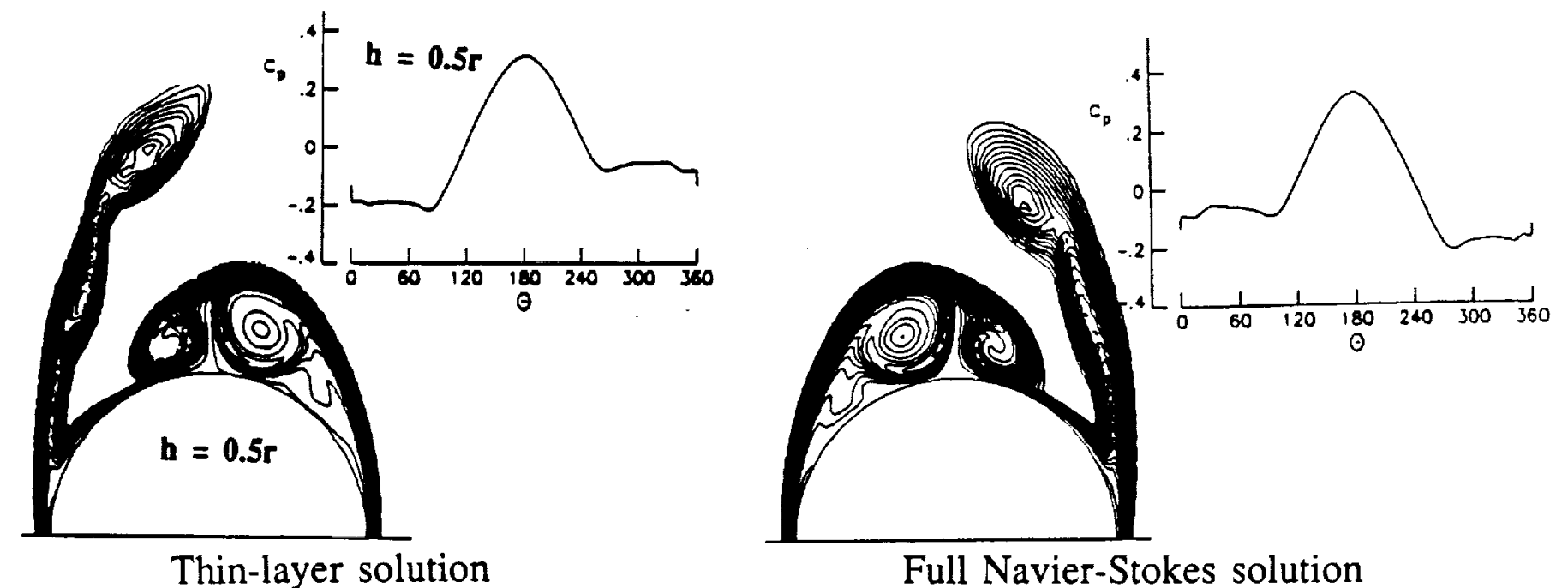

Figure 3. Comparison of passive flow control using a vertical fin, $h=0.5 r$.

$=$

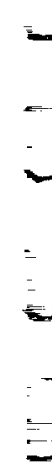

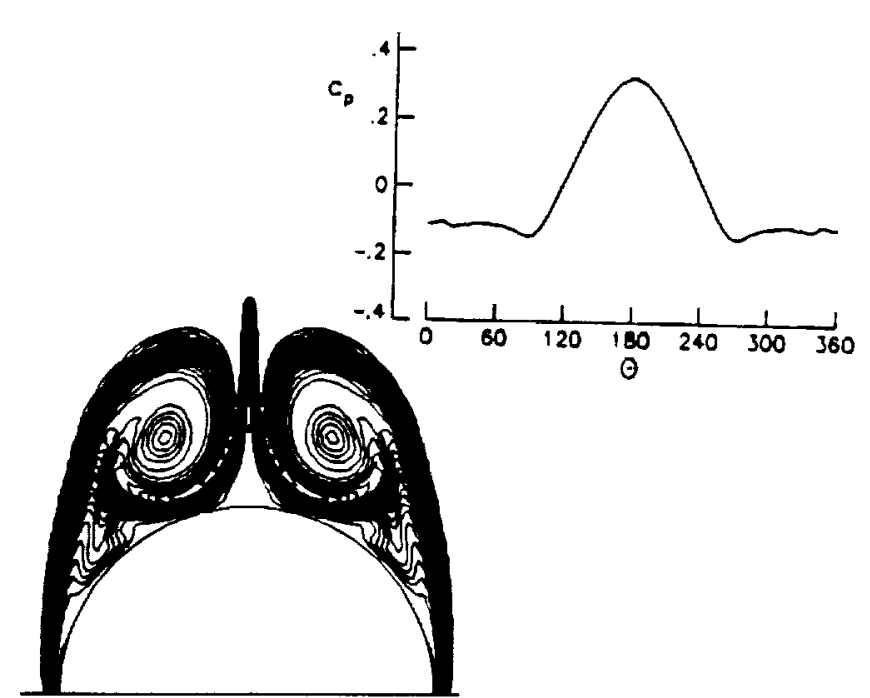

Full Navier-Stokes solution

Figure 4. Comparison of passive flow control using a vertical fin, $h=r$ - 

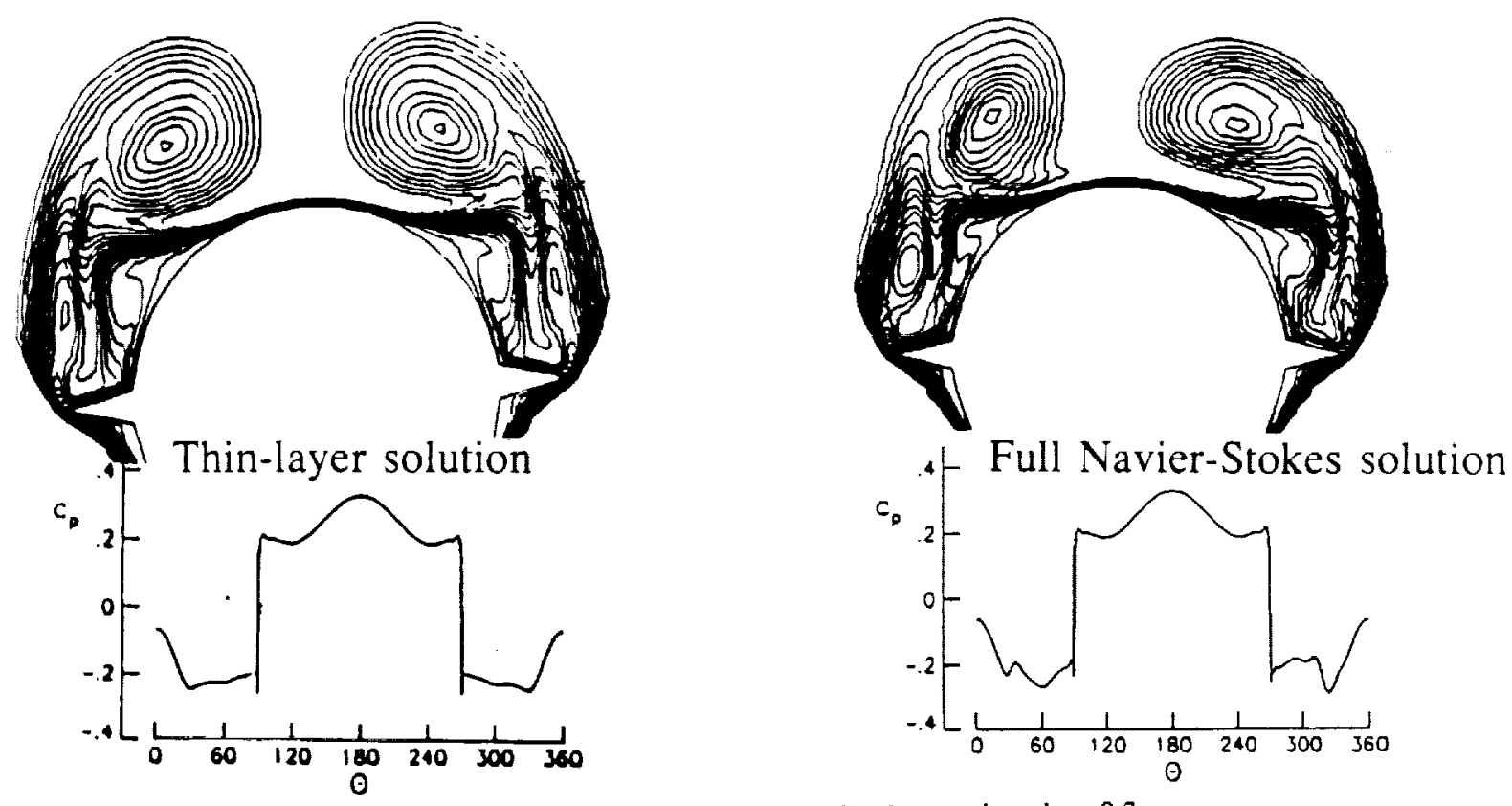

Figure 5. Comparison of passive flow control using strakes, $h=0.3 r$.
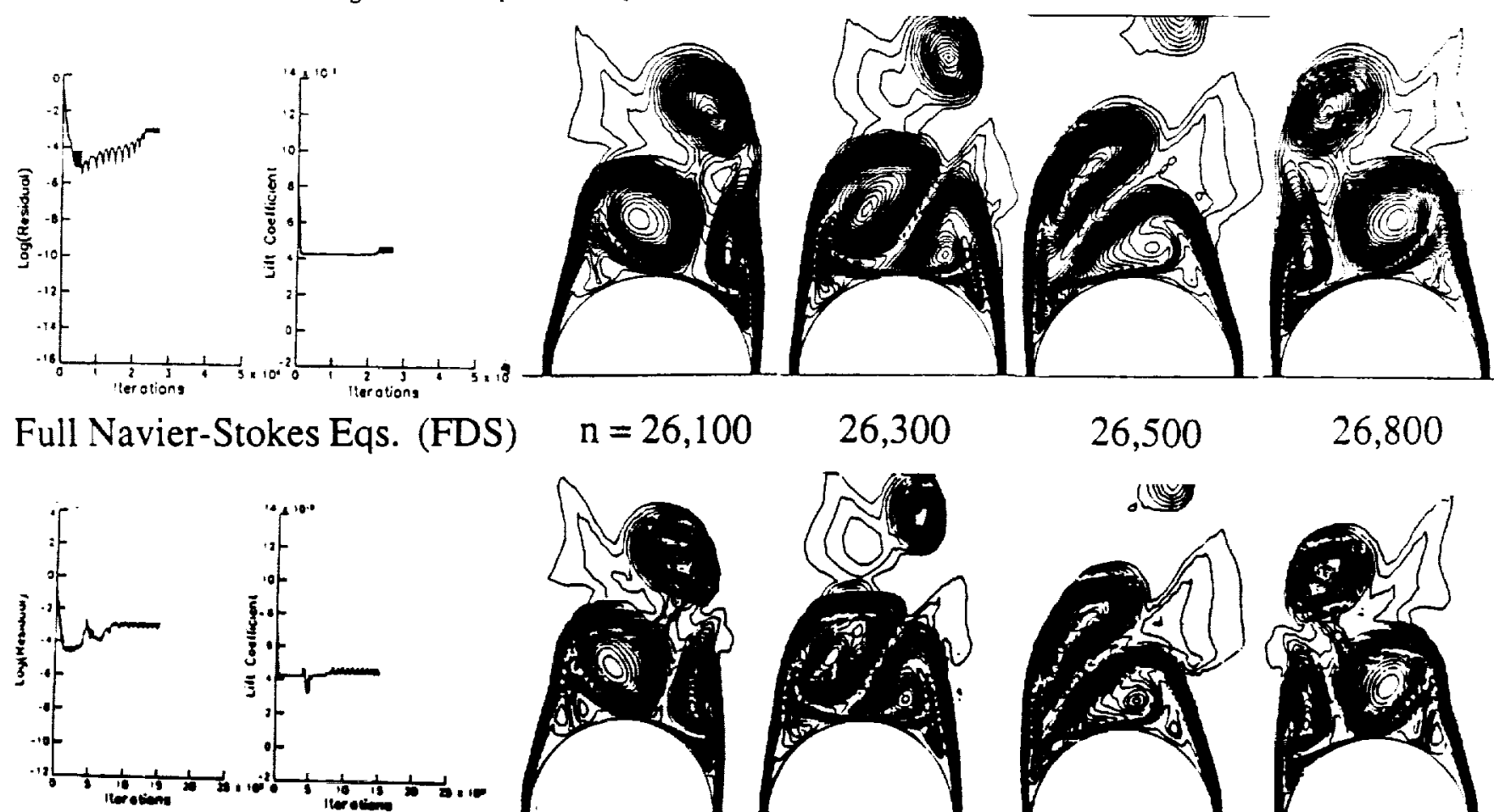

Thin-Layer Eqs. (FDS)
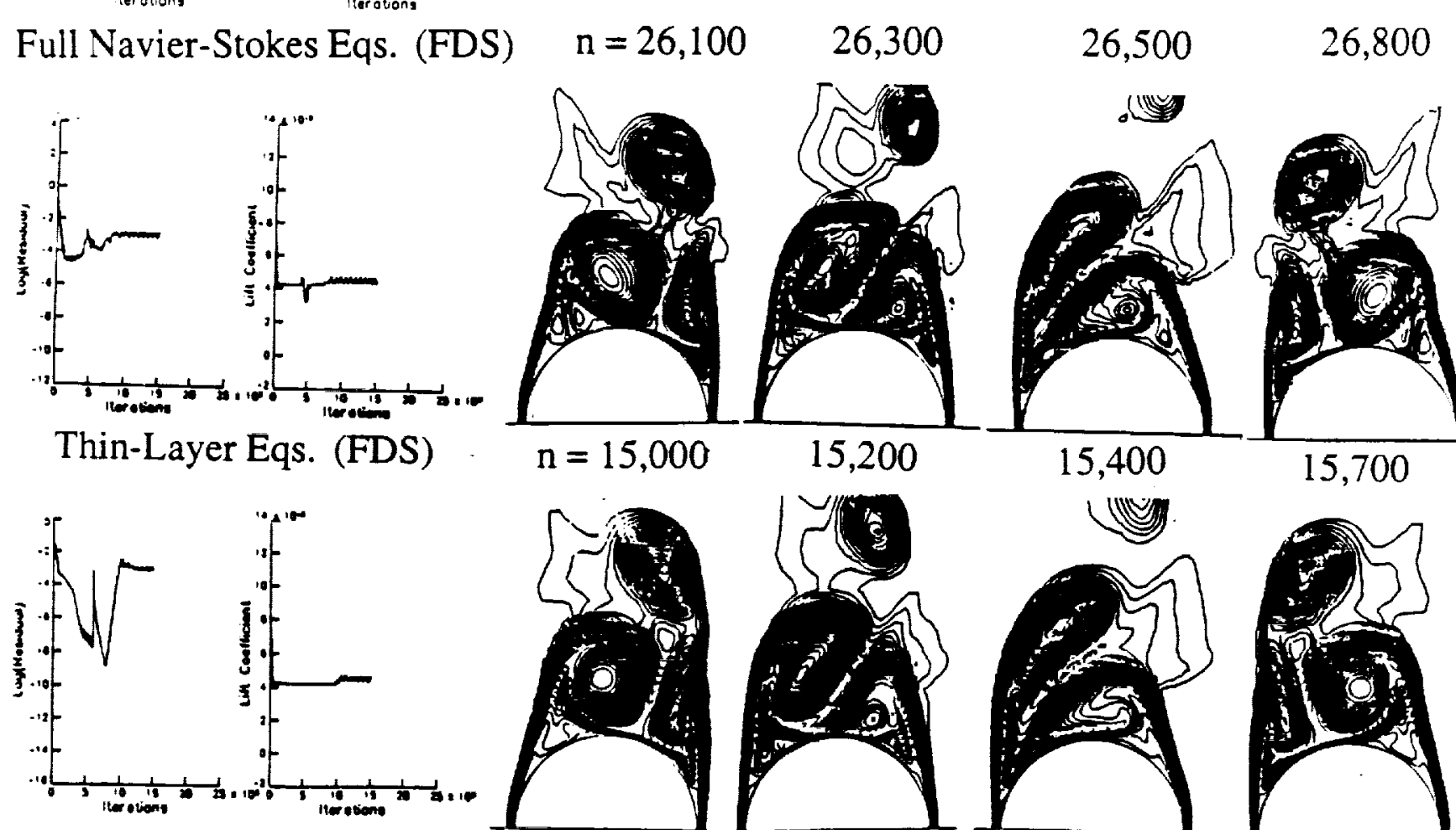

Thin-Layer Eqs. (FVS)
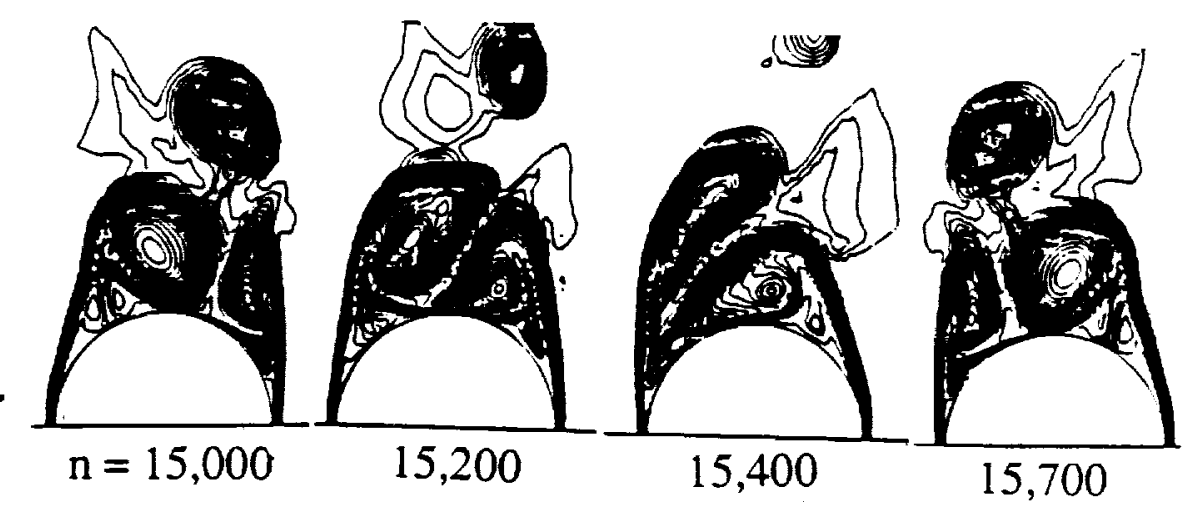

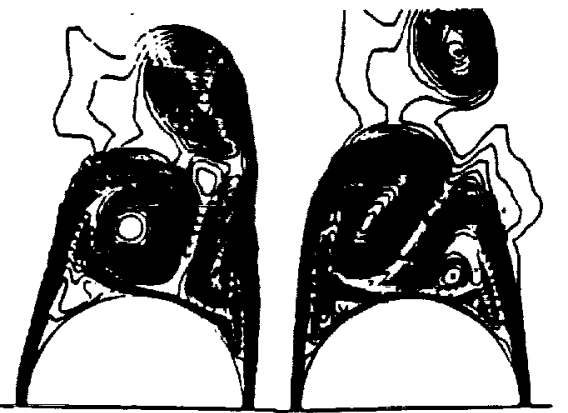

$\mathrm{n}=13,900$

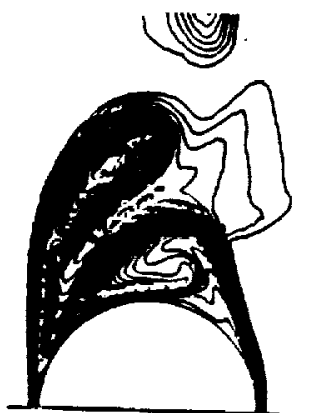

14,300

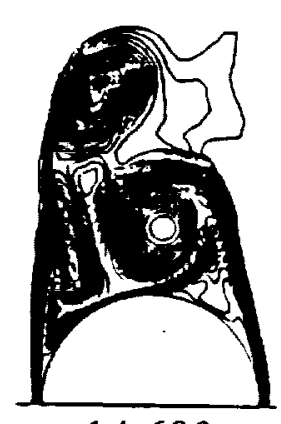

14,600

Figure 6. Comparison of unsteady flow asymmetry using the full Navier-Stokes equations and the thin-layer equations. 

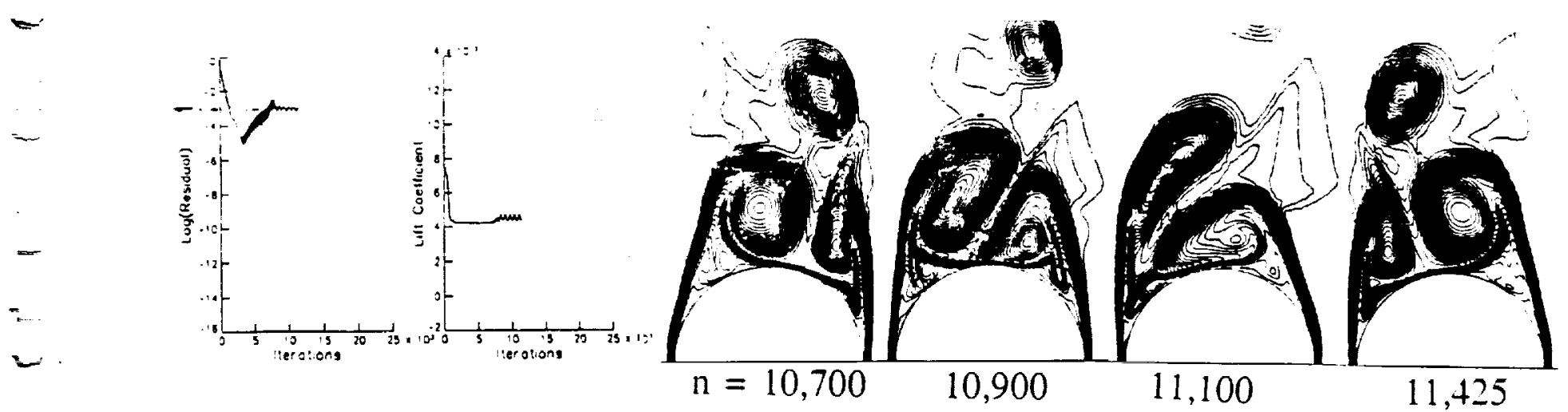

Figure 7. Unsteady flow asymmetry using the full Navier-Stokes equations on a fine grid $241 \times 81$.

Figure 8. Passive flow control using a vertical fin, $h=r$, full Navier-Stokes solution.
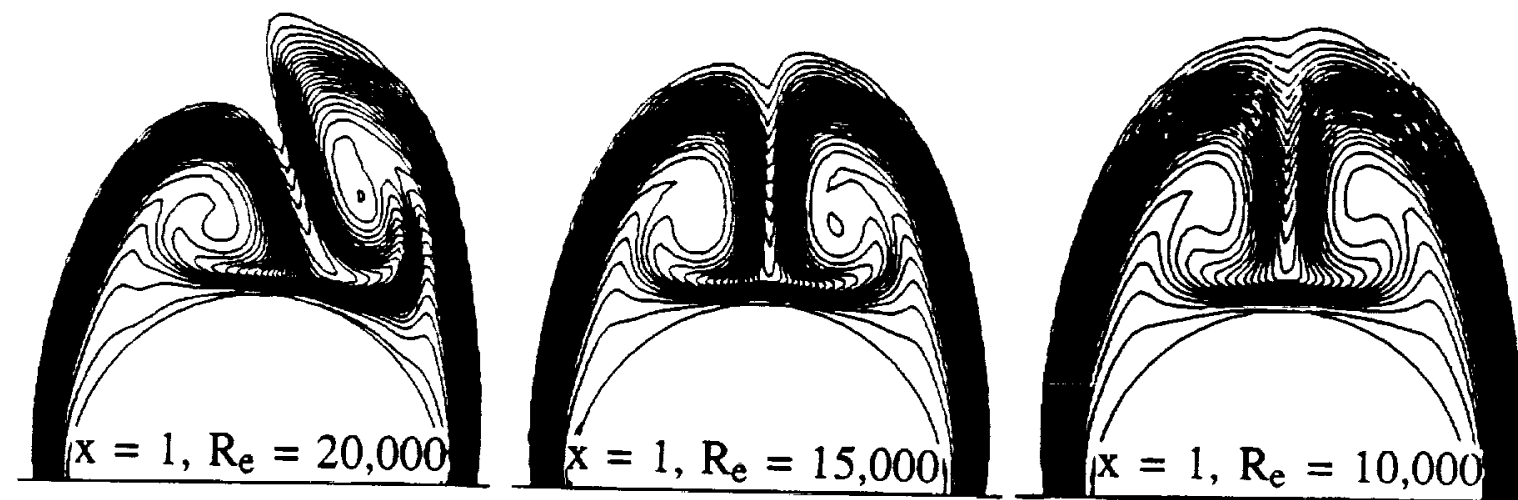

Figure 9. Effect of decreasing the Reynolds number keeping the axial station constant, $x=1,241 \times 81$, full Navier-Stokes solutions.

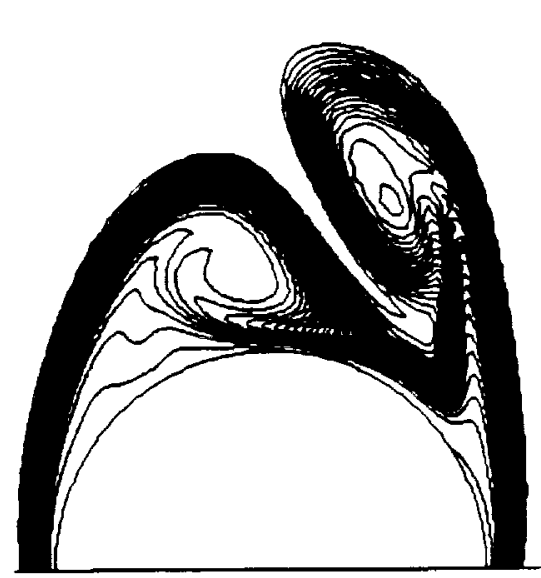

$$
x=0.5, R_{e}=50,000
$$
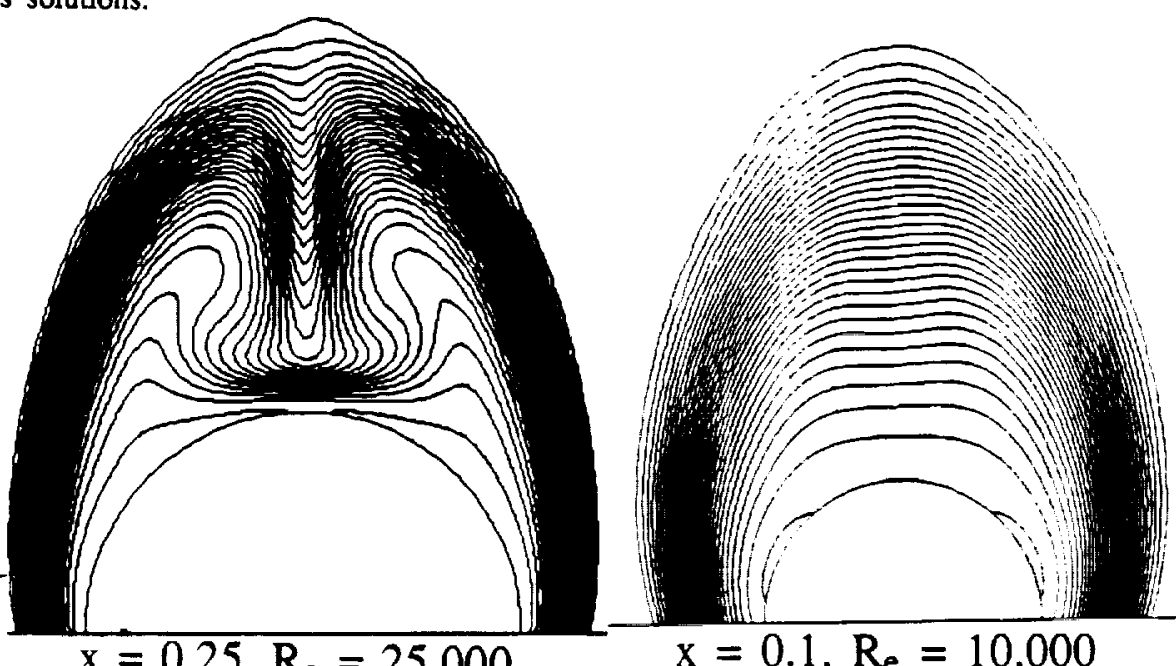

Figure 10. Effect of decreasing the Reynolds number and the axial station, $R_{e}=50,000 ; 25,000 ; 10,000$; $x=0.5,0.25,0.1 ; 241 \times 161$, full Navier-Stokes solutions. 

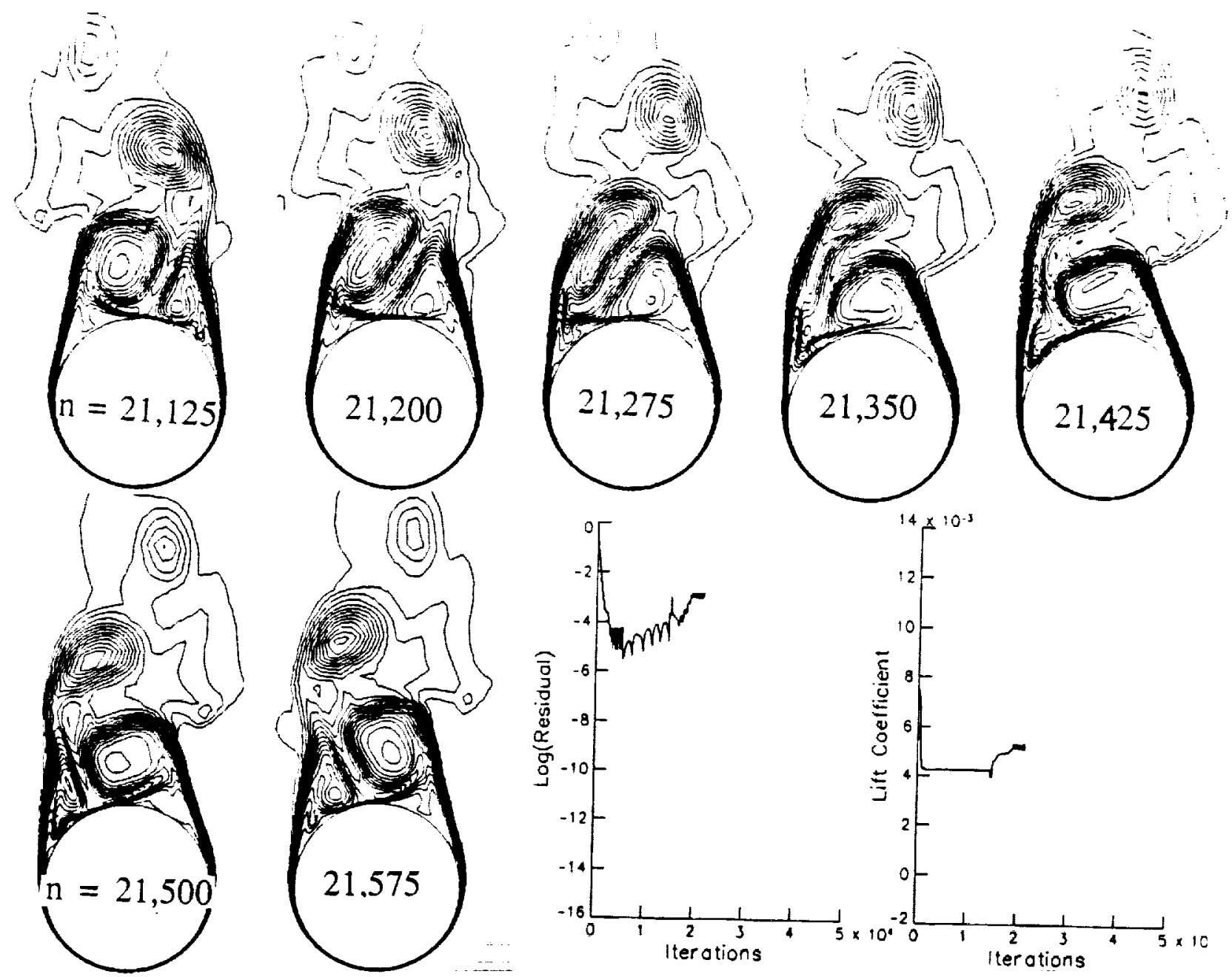
Figure 11. Unsteady flow asymmetry using the full Navier-Stokes equations, $\alpha=35^{\circ}, \mathrm{M}_{\infty}=1.8, \mathrm{R}_{e}$
$=10^{5}$.

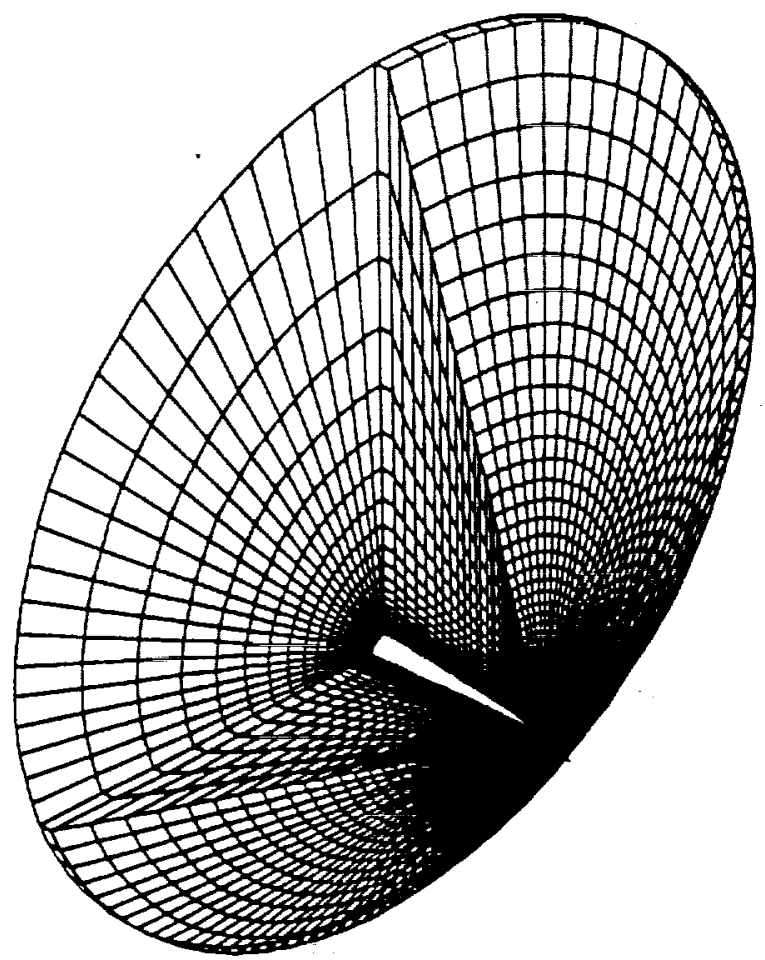

Figure 12. Typical conical grid for a three-dimensional cone, $161 \times 81 \times 65, \Delta \xi_{\min }^{2}=10^{-6}$ at the vertex. 


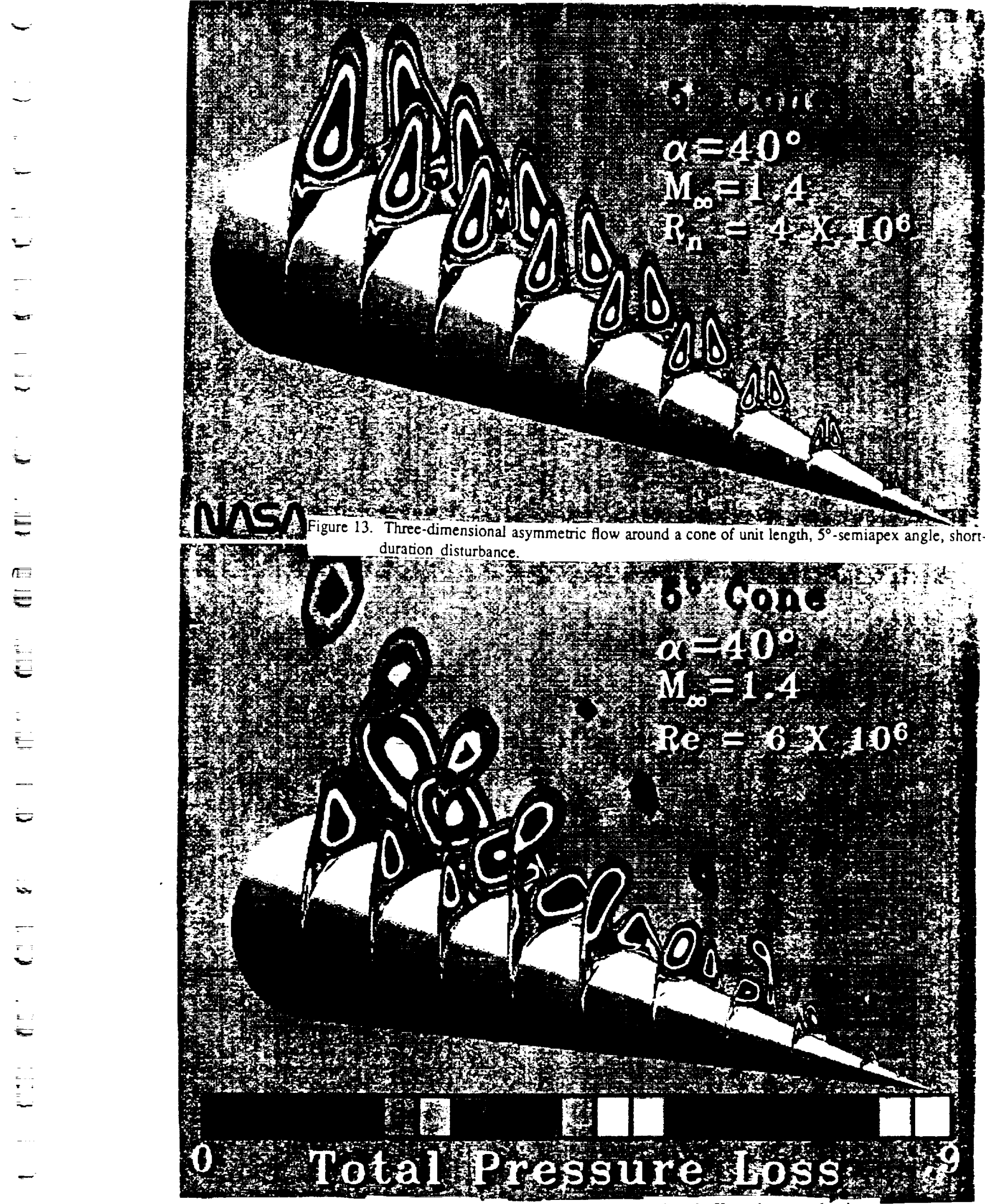

Figure 14. Three-dimenisonal asymmetric flow around a cone of unit length, $5^{\circ}$-semiapex angle, shortduration disturbance. 


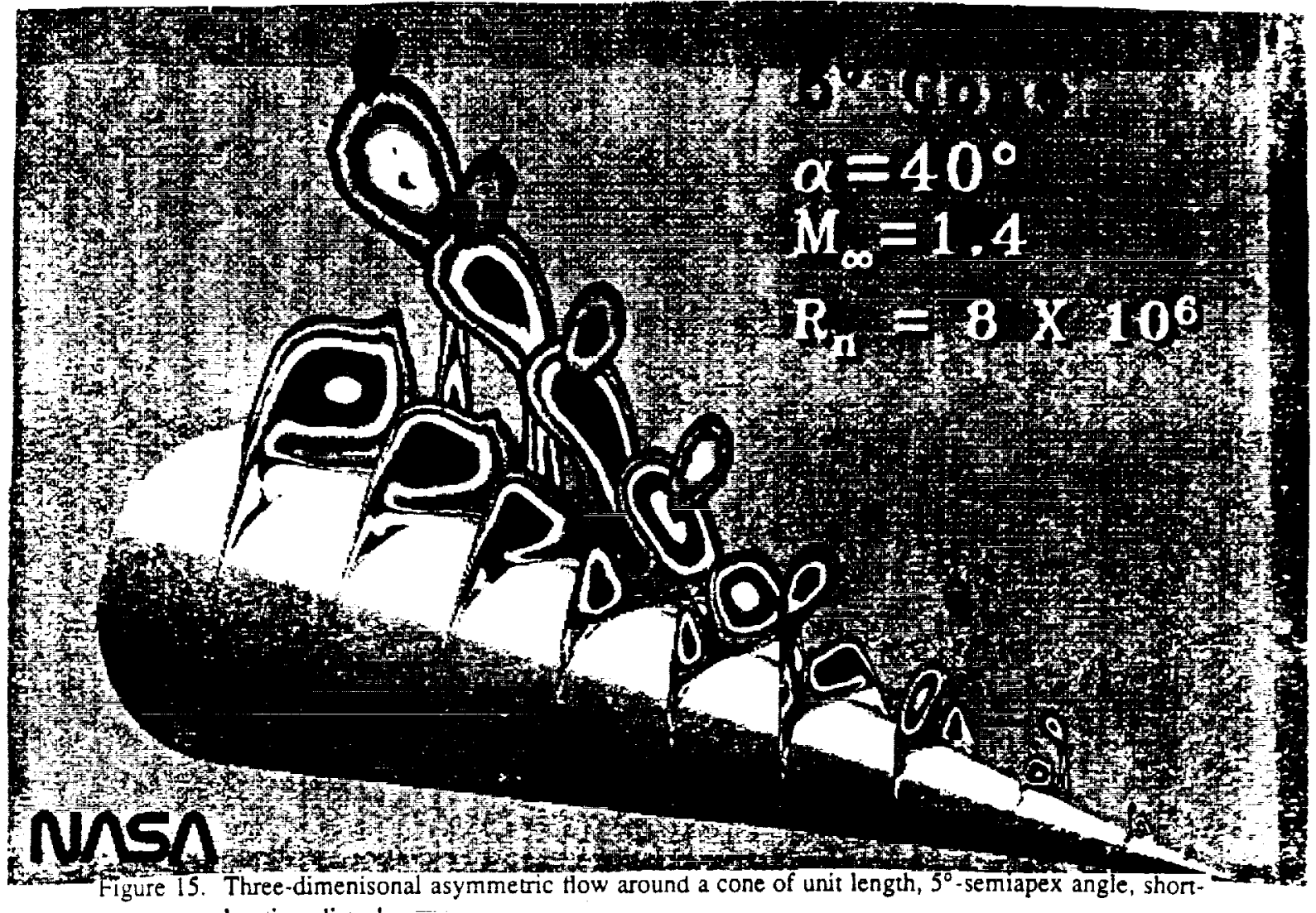
duration disturbance.

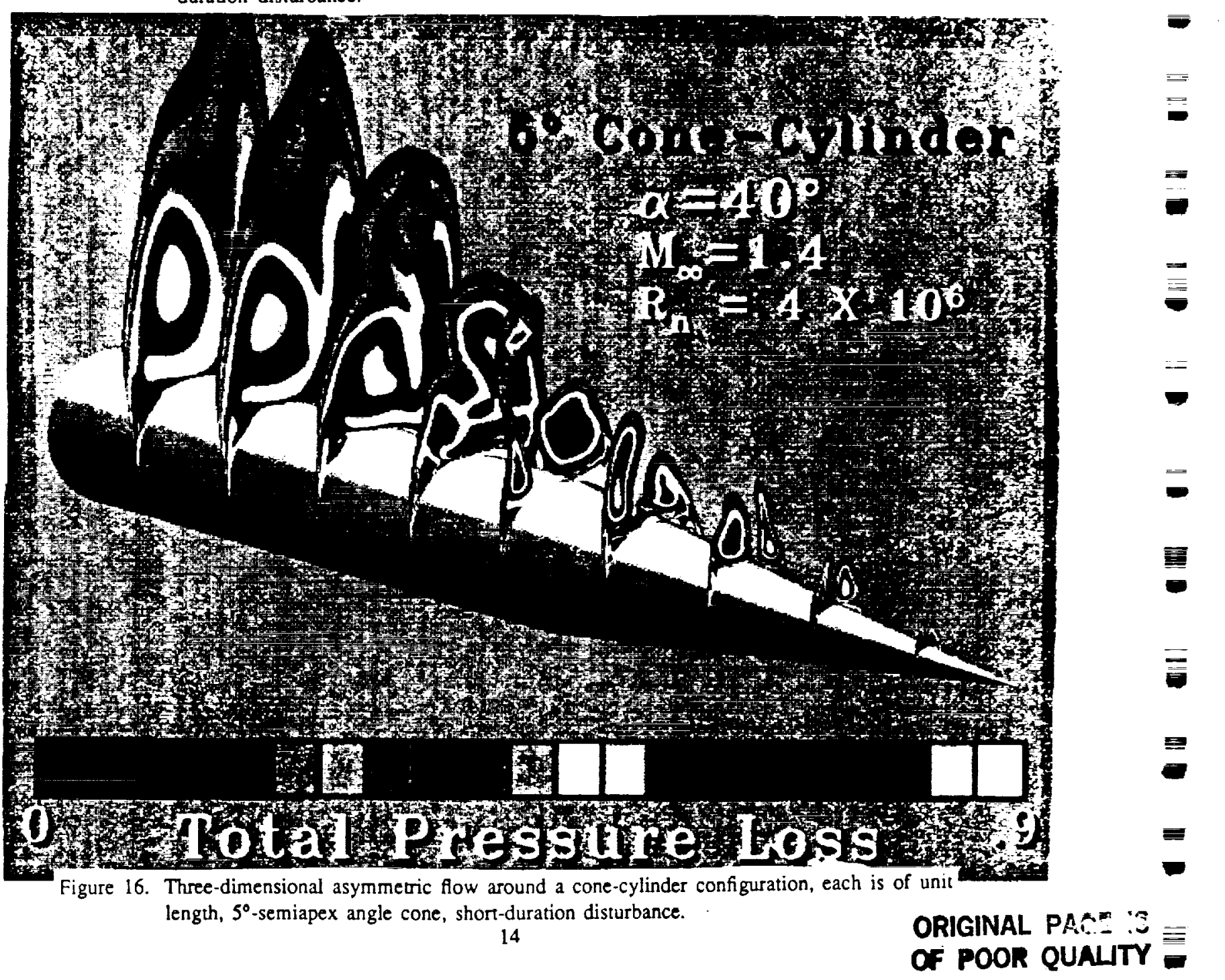



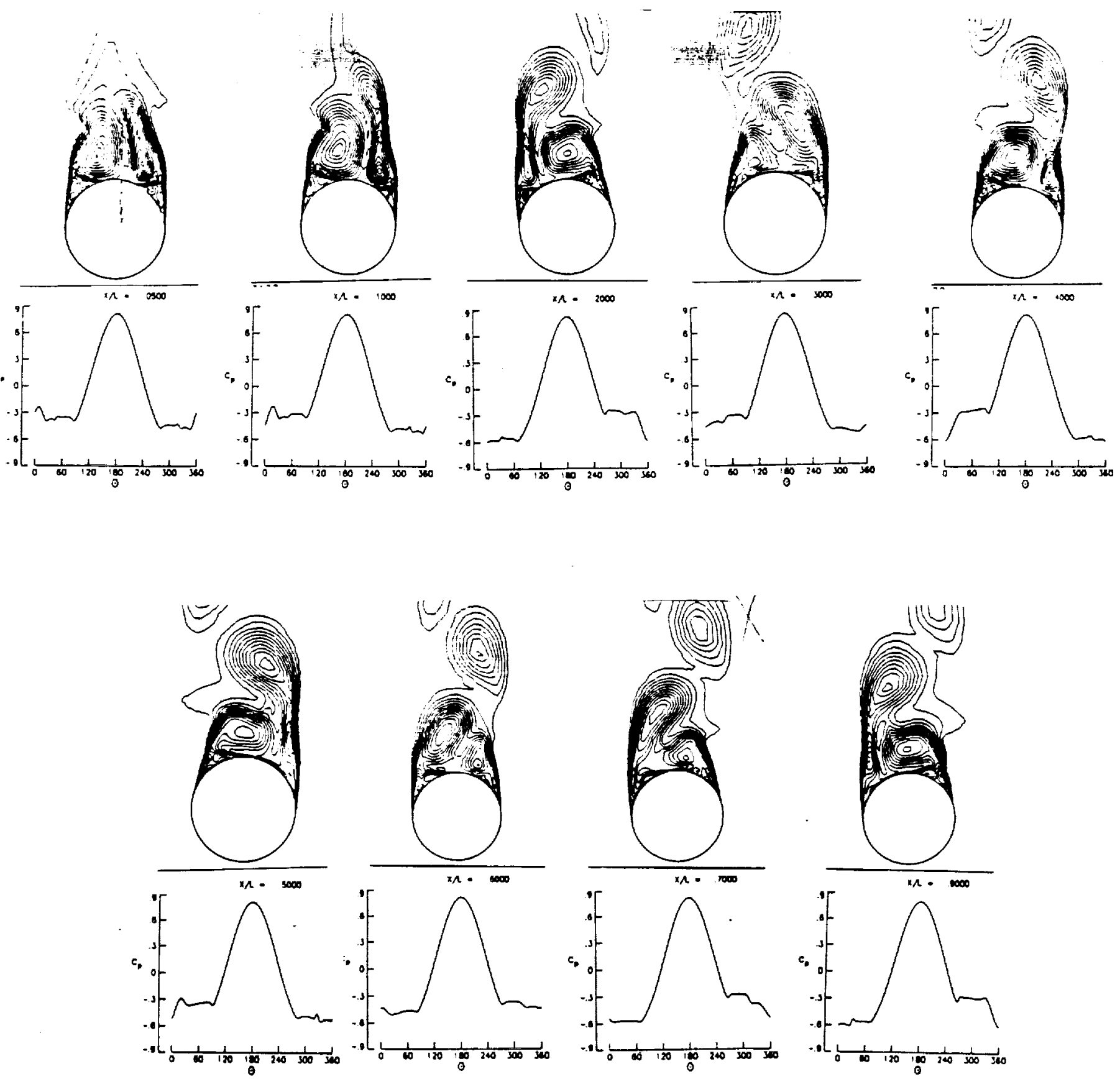

Figure 17. Total-pressure-loss contours and surface-pressure coefficients on cross-flow planes for asymmetric flow around a cone of unit length, $\alpha=40^{\circ}, \mathrm{M}_{\infty}=1.4, \mathrm{R}_{e}=8 \times 10^{6}$. 

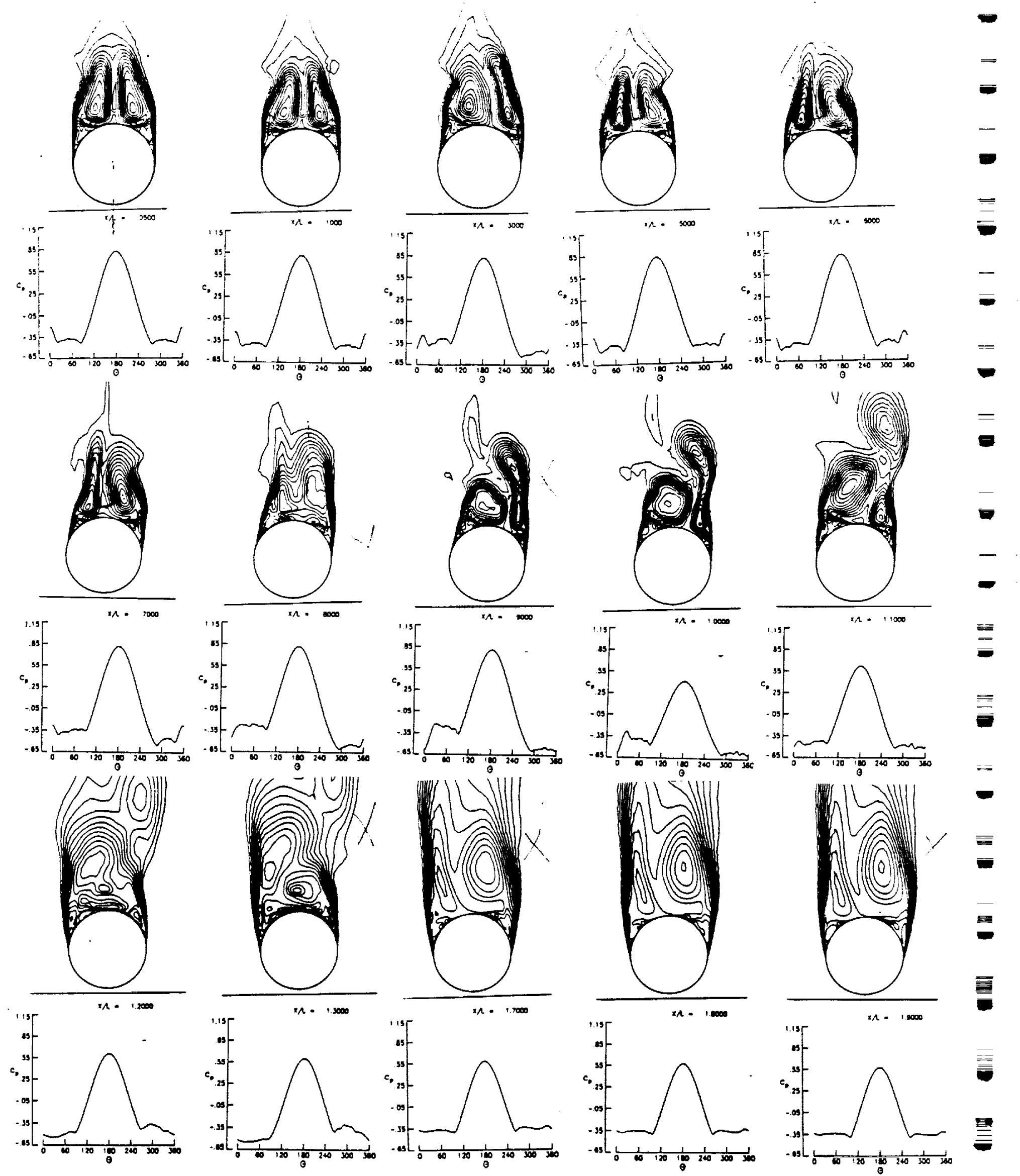

Figure 18. Total-pressure-loss contours and surface-pressure coefficients on cross-flow planes for asymmetric flow around a cone-cylinder configuration, $\alpha=40^{\circ}, \mathrm{M}_{\infty}=1.4, \mathrm{R}_{\mathrm{e}}=4 \times 10^{6}$. 


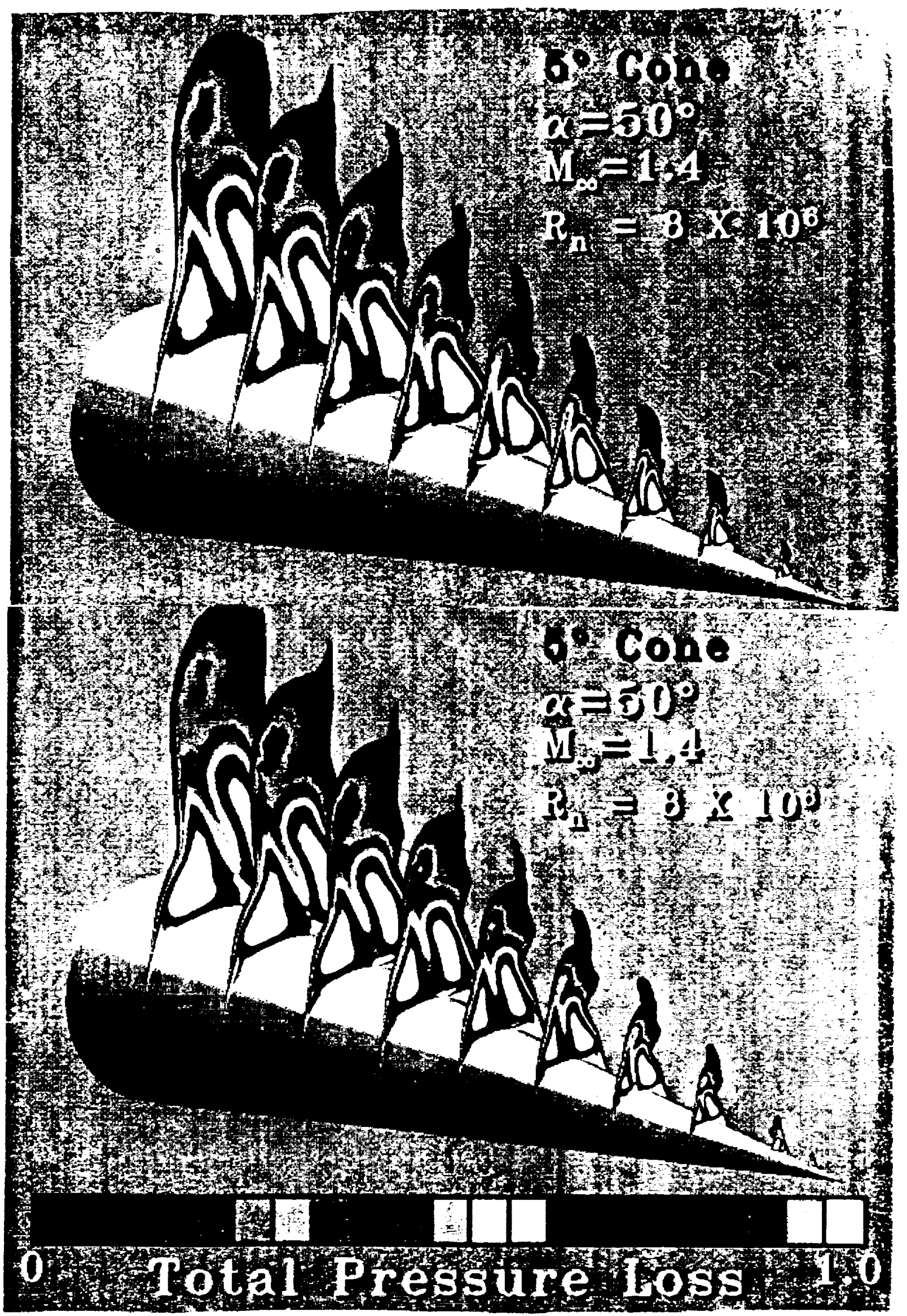

Figure 19. Unsteady three-dimensional asymmetric flow around a cone of unit length, $5^{\circ}$-semiapex angle, $n_{1}=10,116, n_{2}=11,818, \Delta t=10^{-5}$. 



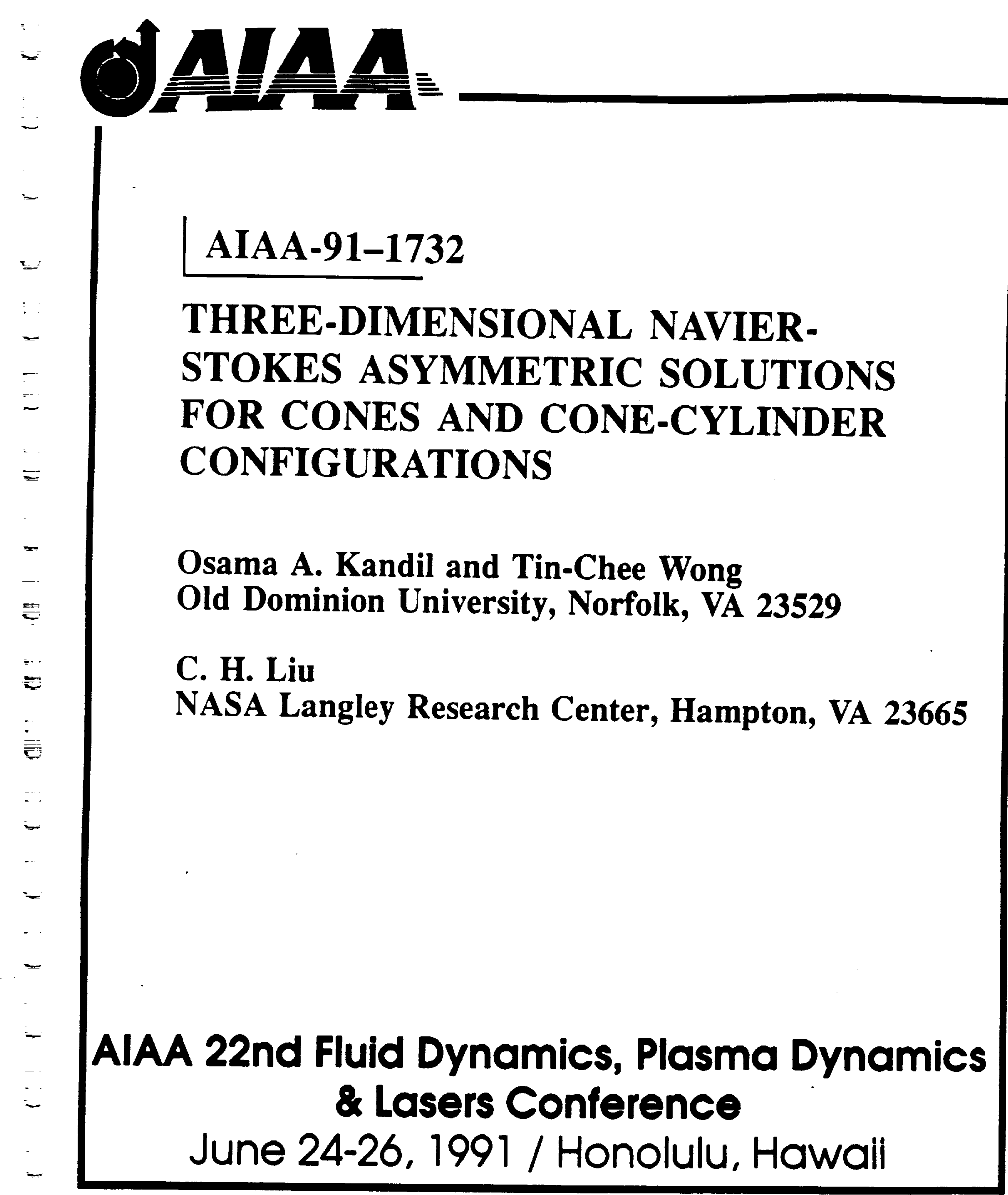

For permiation to copy or republith, contact the Amerken Inattute of Aeronoultics and Aftronoultes 370 L'Entert Fromenads, S.W., Wathington, D.C. 20024 
$\underline{\underline{2}}$

틀

$\bar{\square}$

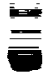

$\equiv$

$-$

$\sigma$

$\overline{\bar{E}}$

흐르

$=$

픔

$\equiv$
$\equiv$

$=$

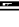

$\bar{\theta}$

$\underline{\underline{\underline{\underline{\underline{n}}}}}$

$=$

- 


\title{
THREE-DIMENSIONAL NAVIER-STOKES ASYMMETRIC SOLUTIONS FOR CONES AND CONE-CYLINDER CONFIGURATIONS
}

\author{
Osama A. Kandil" and Tin-Chee Wong ${ }^{\dagger}$ \\ Old Dominion University, Norfolk, VA 23529 \\ C. H. Liu $^{\ddagger}$ \\ NASA Langley Research Center, Hampton, VA 23665
}

\begin{abstract}
The unsteady, compressible, thin-layer Navier-Stokes equations are used to obtain three-dimensional, asymmetric, vortex-flow solutions around cones and conecylinder configurations. The equations are solved using an implicit, upwind, flux-difference splitting, finitevolume scheme. The computational applications cover asymmetric flows around a $5^{\circ}$ semi-apex angle cone of unit length at various Reynolds number. Next, a cylindrical afterbody of various length is added to the conical forebody to study the effect of the length of cylindrical afterbody on the flow asymmetry. One of the computational solutions has been validated by comparing the computed surface pressure with those of the experimental data. All the asymmetric flow solutions have been obtained by using a short-duration side-slip disturbance.
\end{abstract}

\section{Introduction}

In this paper, the problem of asymmetric vortex-flow around three-dimensional cone and cone-cylinder body is addressed. This problem has received considerable attention by researchers in the computational fluid dynamics area $a^{1.7}$ and by researchers in the experimental fluid dynamics $\operatorname{area}^{6-13}$. The problem is of vital importance to the dynamic stability and controllability of missiles and fighter aircraft. When flow asymmetry develops, it produces side forces, asymmetric lifting forces and corresponding yawing, rolling and pitching moments that might be larger than those available by the control system of the vehicle. The onset of flow asymmetry $\propto c$ curs when the relative incidence (ratio of angle of attack to nose semi-apex angle) of pointed forebodies exceeds certain critical values. At these critical values of relative incidence, flow asymmetry develops due to natural and/or forced disturbances. The origin of natural disturbances may be a transient side-slip, an acoustic disturbance, or similar disturbance of short duration. The origin of forced disturbances is geometric perturbations due to imperfections in the nose geometric symmetry or similar disturbances of permanent nature. In addition to

\footnotetext{
Professor and Eminent Scholar, Depe of Mecharical Engineering and Mecharics, Associate Fellow ALAA.

'Research Assistant Professor, Sume Dept, ALA member. tGroup Leader, Theoredical Flow Physics Branch, Semior member ALAA.

Copyright 1991 by the American Institute of Aeronautica and Astronautics, Inc. All rights reserved.
}

the relative incidence as one of the influential parameters for the onset of flow asymmetry, the freestream Mach number, Reynolds number and shape of the body-cross sectional area are also important parameters.

In several recent papers by the present authors ${ }^{1.4}$, the unsteady, thin-layer, compressible Navier-Stokes equations have been used to simulate steady and unsteady, asymmetric vortex flows, including their passive control, around cones with different cross-sectional shapes. The emphasis of these papers was extensive computational studies of the parameters which influence the asymmetric flow phenomenon and its passive control. Since the computational cost associated with the solution of threedimensional-flow problems with reasonable flow resolution is very expensive, all the computational solutions were obtained using a locally-conical flow assumption. Such an assumption reduces the problem solution to that on two conical planes, which are in close proximity of each other, and hence it reduces the computational cost by an order of magnitude. Moreover, such solutions still provide extensive understanding of the fow physics since one can use very fine grids for reasonable flow resolution. These sudies showed that asymmetric flow solutions were unique irrespective of the type of flow disturbance; a random disturbance in the form of a machine round-off error or a controlled disturbance in the form of a shortduration side-slip disturbance. Unsteady asymmetric flow solutions with perfectly periodic vortex shedding were successfully simulated, and the solutions were unique irrespective of the computational scheme used. We also showed that as the Mach number was increased, the flow asymmetry was decreased and as the Reynolds number was increased, the flow asymmetry was increased. The cross-sectional shape of the cone has been shown to be a very influential parameter on the flow asymmetry. Circular sections produced very strong flow asymmetry and diamond sections produced relatively-weaker flow asymmetry. Passive control of the flow asymmerry was demonstrated by using vertical fins of different heights along the leeward plane of geometric symmetry and by using thin and thick side strakes with different orientations. It was also shown that side-strakes control is more practical than the vertical-fin control since it was effective over a wide range of angle of attack and provided additional lifting force. In a later paper, by the present authors', the full Navier-Stokes solutions were compared with the thin-layer Navier-Stokes solutions. It was shown that the full Navier-Stokes solutions produced thicker free-shear 
layers and more vortex-core resolution as compared with those of the thin-layer Navier-Stokes equations. In reference 5 , a few tentative three-dimensional flow solutions were also presented.

In an attempt to simulate asymmetric vortex flow around an ogive-cylinder body at an angle of attack of $40^{\circ}$, a freestream Mach number of 0.2 and a freestream Reynolds number of 200,000; Degani and Schiff $f^{6}$ used the unsteady, thin-layer, Navier-Stokes equations along with an implicit scheme which is second-order accurate in time. The scheme uses central-differencing in the crossflow plane and upwind flux-vector splitting in the streamwise direction. By introducing a forced asymmetric disturbance near the body nose in the form of a small surface jet, asymmetric flow solution was obtained. When the jet was turned off, the flow asymmetry dissipated and the flow recovered its symmetry.

In a later paper by Degani ${ }^{7}$, the same computational scheme was used to solve for the flow around the same ogive-cylinder body over a wide range of angle of attack; $\alpha=20^{\circ}-80^{\circ}$. His numerical experiments focused on investigating the origin of vortex asymmetry. Based on his results, he suggested that the flowfield around slender bodies could be divided into three main groups depending on the angle of attack range. This range might change by $\pm 10^{\circ}$, depending on the flow conditions. In the range $0^{\circ}<\alpha<30^{\circ}$, the flow was symmetric and introduction of small disturbances near the nose had a small effect on the flow symmetry. In the second range, $30^{\circ}<\alpha<60^{\circ}$, the flow became steady asymmetric upon introduction of a space-fixed forced disturbance near the nose. The level of asymmetry was a function of the location and size of the forced disturbance, and for large size disturbances, the asymmetry became unsteady with very high frequency. However, when the disturbance was removed the flow recovered its symmetric shape. He attributed the origin of asymmetry to a convective-type-instability mechanism. In the very high range, $60^{\circ}<\alpha<80^{\circ}$, the flow became unsteady with vortex shedding upon introduction of a small transient disturbance with short duration. He attributed the origin of flow unsteadiness and vortex shedding to an absolute-type-instability mechanism. In that range of angle of attack, he also showed that the convective-typeinstability mechanism was possible upon introduction of a space-fixed disturbance near the nose. Although this investigation revealed good tentative conclusions, there are several remaining questions to be addressed, which are related to the scheme dissipative effects, particularly in the cross-flow planes, and the grid fineness and its resolution of the disturbance growth.

In the present paper, we focus on the threedimensional asymmetric flow problem. In particular, we address several important issues concerning the flow asymmetry around three-dimensional bodies. First, the three-dimensional asymmetric flow around a $5^{\circ}$ semi-apex angle cone of unit length is considered in response to a short-duration disturbance in the form of a transient side slip. With guidance from the locally-conical solutions, the angle of attack is varied between $30^{\circ}$ and $50^{\circ}$, the Mach number is varied between 1.4 and 1.8 and the Reynolds number is varied between $10^{5}$ and $8 \times 10^{8}$ searching for asymmetric flow solutions due to a short-duration disturbance. Next, the flow conditions are fixed and a cylindrical afterbody is added to the same cone of unit length to study the effect of the length of the cylindrical afterbody. The computational results have also been verified using comparison of the surface-pressure coefficient with that of the experimental data of Landrum ${ }^{8}$.

\section{Formulation}

\section{Thin-Layer Navier-Stokes Equations}

The conservative form of the dimensionless, unsteady, compressible, thin-layer Navier-Stokes equations in terms of time-independent, body-conformed coordinates $\xi^{1}, \xi^{2}$ and $\xi^{3}$ is given by

$$
\frac{\partial \bar{Q}}{\partial \mathrm{t}}+\frac{\partial \overline{\mathrm{E}}_{\mathrm{s}}}{\partial \xi^{\mathrm{s}}}-\frac{\partial\left(\overline{\mathrm{E}}_{\mathrm{v}}\right)_{2}}{\partial \xi^{2}}=0 ; \mathrm{s}=1-3
$$

where

$$
\begin{gathered}
\xi^{\mathrm{m}}=\xi^{\mathrm{m}}\left(\mathrm{x}_{1}, \mathrm{x}_{2}, \mathrm{x}_{3}\right) \\
\overline{\mathrm{Q}}=\frac{\hat{\mathrm{q}}}{\mathrm{J}}=\frac{1}{\mathrm{~J}}\left[\rho, \rho \mathrm{u}_{1}, \rho \mathrm{u}_{2}, \rho \mathrm{u}_{3}, \rho \mathrm{e}\right]^{\mathrm{t}} \\
\overline{\mathrm{E}}_{\mathrm{m}} \equiv \text { inviscid flux } \\
=\frac{1}{\mathrm{~J}}\left[\partial \xi^{\mathrm{m}} \hat{\mathrm{E}}_{\mathrm{k}}\right]^{\mathrm{t}} \\
=\frac{1}{\mathrm{~J}}\left[\rho \mathrm{U}_{\mathrm{m}}, \rho \mathrm{u}_{1} \mathrm{U}_{\mathrm{m}}+\partial_{1} \xi^{\mathrm{m}} \mathrm{p}, \rho \mathrm{u}_{2} \mathrm{U}_{\mathrm{m}}\right. \\
\left.+\partial_{2} \xi^{\mathrm{m}} \mathrm{p}, \rho \mathrm{u}_{3} \mathrm{U}_{\mathrm{m}}+\partial_{3} \xi^{\mathrm{m}} p,(\rho \mathrm{e}+\mathrm{p}) \mathrm{U}_{\mathrm{m}}\right]^{\mathrm{t}}
\end{gathered}
$$

$$
\begin{aligned}
& \left(\bar{E}_{\mathrm{v}}\right)_{2} \equiv \text { viscous and heat-conduction flux in } \xi^{2}
\end{aligned}
$$

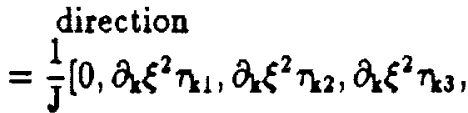

$$
\begin{aligned}
& \left.\partial_{k} \xi^{2}\left(u_{n} \eta_{n}-q_{k}\right)\right]^{t} ; \quad k=1-3, n=1-3 \\
& \mathrm{U}_{\mathrm{m}}=\partial_{\mathrm{k}} \xi^{\mathrm{m}} \mathrm{u}_{\mathrm{k}}
\end{aligned}
$$

The first element of the three momentum elements of Eq. (5) is given by

$$
\partial_{k} \xi^{\mathbf{s}} n_{1} \equiv \frac{\mathbf{M}_{x \mu} \mu}{\operatorname{Re}}\left(\psi \partial_{1} \xi^{2}+\phi \frac{\partial u_{1}}{\partial \xi^{2}}\right)
$$

The second and third elements of the momentum elements are obtained by replacing the subscript 1 , everywhere in 
Eq. (7), with 2 and 3, respectively. The last element of Eq. (5) is given by

$$
\begin{aligned}
\partial_{k} \xi^{2}\left(u_{n} \pi_{\mathrm{n}}\right. & \left.-q_{k}\right) \equiv \frac{\mathrm{M}_{x} \mu}{\operatorname{Re}}\{\psi \mathrm{W} \\
& +\phi\left[\frac{1}{2} \frac{\partial}{\partial \xi^{2}}\left(u_{\mathrm{p}} u_{\mathrm{p}}\right)\right. \\
& \left.\left.+\frac{1}{(\gamma-1) \mathrm{Pr}_{\mathrm{r}}} \frac{\partial\left(\mathrm{a}^{2}\right)}{\partial \xi^{2}}\right]\right\} ; \mathrm{p}=1-3
\end{aligned}
$$

where

$$
\phi=\partial_{k} \xi^{2} \partial_{k} \xi^{2}, \psi=\frac{1}{3} \partial_{k} \xi^{2} \frac{\partial u_{k}}{\partial \xi^{2}}, W=\partial_{n} \xi^{2} u_{n}
$$

In Eqs. (1)-(9), the dimensionless variables are referenced to their appropriate freestream values. The dimensionless density $\rho$, Cartesian velocity components $u_{1}, u_{2}$ and $u_{3}$, total energy per unit mass, e, dynamic viscosity, $\mu$ and speed of sound, a, are defined as the ratio of the corresponding physical quantities to those of the freestream; namely, $\rho_{x}, a_{x}, \rho_{x} a_{x}^{2}, \mu_{x}$ and $a_{x}$; respectively. The pressure, $\mathrm{p}$, is non-dimensionalized by $\rho_{\propto} a_{\infty}^{2}$, and is related to the total energy for a perfect gas by the equation of state

$$
\bar{p}=(\gamma-1) \rho\left(e-\frac{1}{2} u_{j} u_{j}\right) ; j=1-3
$$

where $\gamma$ is the ratio of specific heats and its value is 1.4 . The viscosity, $\mu$, is calculated from the Sutherland's law

$$
\mu=\mathrm{T}^{3 / 2}\left(\frac{1+\mathrm{c}}{\mathrm{T}+\mathrm{c}}\right), \mathrm{c}=0.4317
$$

where $\mathrm{T}$ is the temperature which is non-dimensionalized by $\mathrm{T}_{x}$. The Prandul number, $\mathrm{P}_{\mathrm{r}}$ is fixed at 0.72 . The Reynolds number is defined as $R_{e}=\rho_{\infty} \mathrm{U}_{\infty} \mathrm{L} / \mu_{\infty}$ and the characteristic length, $L$, is chosen as the length of the body.

In Eqs. (1)-(10), the indicial notation is used for convenience. The subscripts $k, n, p$ and $j$ are summation indices, the superscript or subscript $s$ is a summation index and the superscript or subscript $m$ is a free index. The partial derivative $\frac{\partial}{\partial x_{k}}$ is referred to by $\partial_{k}$.

\section{Boundary and Initial Conditions}

Boundary conditions are explicitly implemented. They include the inflow-outflow conditions and the solid-boundary conditions. At the plane of geometric symmetry, periodic conditions are used. Since the freestream Mach number is supersonic and the inflowoutflow boundaries are also supersonic, freestream conditions are specified at the inflow boundaries and first-order extrapolation of the flow variables is used at the outflow boundaries. The conical shock enclosing the body is captured as part of the solution. On the solid boundary, the no-slip and no-penetration conditions are enforced; $u_{1}=$ $\mathrm{u}_{2}=\mathrm{u}_{3}=0$, and the normal pressure gradient is set equal to zero. For the temperature, the adiabatic boundary condition is enforced at the solid boundary,

The initial conditions correspond to the freestream conditions with $u_{1}=u_{2}=u_{3}=0$ on the solid boundary. The freestream conditions are given by

$$
\begin{aligned}
& \rho_{x}=\mathrm{a}_{x}=\mathrm{T}_{x}=1, \\
& \mathrm{u}_{1 x}=\mathrm{M}_{x} \cos \alpha \cos \beta, \\
& \mathrm{u}_{2 x}=-\mathrm{M}_{x} \sin \beta, \\
& \mathrm{u}_{3 x}=\mathrm{M}_{x} \sin \alpha \cos \beta, \\
& \mathrm{p}_{x}=1 / \gamma, \mathrm{e}_{x}=\frac{1}{\gamma(\gamma-1)}+\frac{\mathrm{M}_{x}^{2}}{2}
\end{aligned}
$$

where $\alpha$ is the angle of attack and $\beta$ the side slip angle.

\section{Computational Scheme}

The implicit, upwind, flux-difference splitting finitevolume scheme is used to solve the unsteady, compressible, thin-layer Navier-Stokes equations. The scheme uses the flux-difference splitting scheme of Roe which is based on the solution of the approximate Riemann problem. In the Roe scheme, the inviscid flux difference at the interface of computational cells is split into two parts; left and right flux differences. The splitting is accomplished according to the signs of the eigenvalues of the Roe averaged-Jacobian matrix of the inviscid fluxes at the cell interface. The min-mod flux limiter is used to eliminate oscillations in the shock region. The viscousand heat-flux terms are linearized and the cross-derivative terms are eliminated in the implicit operator. The viscous terms are differenced using a second-order accurate central differencing. The resulting difference equation is approximately factored and is solved in three sweeps in the $\xi^{1}, \xi^{2}$ and $\xi^{3}$ directions. The computational scheme is coded in the computer program "CFL3D."

\section{Computational Applications and Discussions}

In the present computational applications, we consider the three-dimensional solutions of the unsteady, compressible, thin-layer Navier-Stokes equations for asymmetric vortex flows around a circular cone and circular cone-cylinder configurations. There are several issues to be addressed through the present study. First, for the same circular cone of $5^{\circ}$-semi-apex angle ${ }^{l}$ and for the same flow conditions and source of disturbance, will the three-dimensional flow solution be the same as that of the locally-conical flow solution? If the answer is negative, the next question to address is: Is there a length scale which relates the three-dimensional solution to the locally-conical solution? The second issue to be addressed is the effect of Reynolds number on the flow asymmetry? The third issue to be addressed is the effect of the cylindrical-afterbody length on the flow asymmetry. Finally, we address the question of code and grid validation by comparing the results of an asymmetric flow solution with those of the experimental data'. 


\section{Circular Cone}

A $5^{\circ}$-semi-apex angle circular cone of unit length (cone length is the characteristic length) is considered to address the first two issues mentioned above. This is the same circular cone which was considered by the authors in Ref. 1 for the locally-conical flow solutions. A threedimensional grid of $161 \times 81 \times 65$ in the wrap around, normal and axial directions, respectively, is generated by using a modified Joukowski transformation at axial stations. The grid is clustered algebraically in the normal direction of the body using a geometric series with minimum grid spacing of $10^{-6}$ at the cone vertex and $10^{-5}$ at the axial station of unit length. A typical grid is shown in Fig. 1. The cross-flow grid size of $161 \times 81$ is the same grid size which was used for the locally-conical flow solutions of Ref. 1.

With the flow conditions set at $\alpha=20^{\circ}, M_{x}=1.8$ and $R_{\mathrm{e}}=10^{5}$, which are the same conditions as those of the locally-conical flow of Ref. 1, the three-dimensional solution produces a symmetric steady flow, unlike the locallyconical solution which produces asymmetric steady flow. The reason for the difference is well understood since the locally-conical solution is obtained at an axial station of $x=1.0$. Hence, a length scale is involved in the Reynolds number, as can be seen from the analytical conical equation for steady viscous flow. Next, the search is directed at obtaining asymmetric flow solutions for the three-dimensional cone flow. In Fig. 2, we show the solution in the form of total-pressure loss for the same cone at $\alpha=40^{\circ}, M_{x}=1.4$ and $R_{e}=4 \times 10^{6}$. It is seen that the solution is asymmetric and is nearly self-similar over a long axial distance of the cone length. This solution is obtained using a short-duration side-slip disturbance. When the residual error drops four orders of magnitude, a side-slip disturbance of $\beta=2^{\circ}$ is applied for 100 iteration steps, then it is removed. Thereafter, the pseudo time stepping is continued until the residual error drops again four to five orders of magnitude and a stable asymmetric solution is obtained. It should be noted that a machine round-off error type of random disturbance cannot be used to obtain three-dimensional asymmetric solutions since the residual error never drops to machine zero.

Next, the Reynolds number is increased to $5 \times 10^{6}$ and $6 \times 10^{6}$ keeping the other flow conditions constant at $\alpha=40$ and $M_{\infty}$ 1.4. Figures 3 and 4 show the totalpressure-loss solutions for these cases. Figure 3 shows that the asymmetry of the vortical flow gets strong and the self similarity of the flow asymmetry is substantially lost. However, it is noticed that the flow asymmetry does not change sides as the solution develops in the downstream direction. Figure 6, which corresponds to the $R_{0}$ of $6 \times 10^{6}$, shows that the flow asymmetry changes sides as the solution develops in the downstream direction. Moreover, it is noticed that shed vortices exist in the flow. A close study of the solutions between the shown fourth cross-flow plane and seventh cross-flow plane reveals that the flow asymmetry changes from the right side (fourth cross-flow plane) to the left side (seventh crossflow plane). The solutions on these two planes are nearly scaled mirror-images of each other. The present spatial flow asymmetry is qualitatively similar to the temporal flow asymmetry of the locally-conical flow solution of Ref. 1 (see Figure 8 of the present paper).

Figures 5 and 6 show front and rear side views of the limiting streamlines for the cases of $R_{e}=4 \times 10^{6}$ and $R_{c}=6 \times 10^{6}$, respectively. By comparing the streamlines, lines of separation and attachment of the front and rear sideviews in each figure, it is noticed that the flow asymmetry exists on the boundary and becomes stronger as the Reynolds number increases. As the Reynolds number increases, the separation and reattachment lines changes from radial straight lines to highly curved lines.

Figure 7 shows the total-pressure-loss solution for the same cone for a higher Reynolds number, $R_{e}=8 \times 10^{6}$. The asymmetry of the vortex flow becomes much stronger as compared with the previous cases of Figs. 2-4. By comparing the solution of this case with that of the $R_{e}$ $=6 \times 10^{6}$, it is noticed the flow asymmetry of the case with high $R_{e}$ changes sides along a shorter axial distance (third and fifth cross-flow planes) in comparison with that of the low $R_{e}$. Moreover, the flow asymmetry of the case with high $R_{8}$ changes sides one more time (fifth and ninth cross-flow planes) and thus a complete wave length of flow asymmetry is formed between the third and ninth cross-flow planes. Strong spatial shed vortex exists in the flowfield. This solution is strongly similar to the unsteady asymmetry local-conical flow solution at different time steps which is depicted in Fig. 8 on a cylinder with the axis of the cylinder representing time. The behavior of the flow asymmetry over one period in Fig. 8 is qualitatively similar to the behavior of the flow asymmetry over one wave length in Fig. 7. Figure 9 shows the total-pressureloss contours and surface-pressure coefficient at different axial stations for the case of Fig. 7. The solutions at axial stations of $\mathrm{X} / \mathrm{L}=0.2$ and 0.9 are almost the same (the total pressure losses are drawn to a scale given by the ratio of the circular diameters at $X / L=1$ station and the local axial station). The flow asymmetry between these two stations represents a full wave length.

\section{Circular Cone-Cylinder Configurations}

To address the issue of the effect of cylindrical afterbody length on the flow asymmetry a cylindrical afterbody of different lengths is added to the unit-length conical forebody. The flow around the resulting conecylinder configurations is solved with the flow conditions of $\alpha=40, M_{\infty}=1.4$ and $R_{e}=4 \times 10^{6}$, which are the same flow conditions of the isolated unit-length cone of Fig. 2. The lengths of the cylindrical afterbody are chosen as $1,1.5$ and 2 and the corresponding grid sizes are taken as $161 \times 81 \times 65,161 \times 81 \times 69$ and $161 \times 81 \times 72$; respectively. The source of flow disturbance is the same short duration $2^{\circ}$-side-slip disturbance. The computed total-pressure loss for these cases are given in Figs. 10. 
11 and 12; respectively. For the cone-cylinder configuration of 1:1 (cone length: cylinder length), Fig. 10 shows a very strong asymmetric flow on the cone, in comparison with the flow asymmetry of the isolated cone of Fig. 2, and on the cylindrical afterbody as well. It should be noted that inside the conical shock surrounding the cone-cylinder configuration, subsonic flow regions exist and hence the downstream cylindrical-afterbody boundary has an upstream effect. The cylindrical afterbody has dual effects which increases the flow asymmetry, the first is due to the cone-cylinder juncture and the second is due to the increase of the local angle of attack of the leeward side of the cylinder. Both of these effects increase the spatial growth of the flow asymmetry.

For the cone-cylinder configuration of 1:1.5, Fig. 11 shows stronger forebody asymmetry (first five cross-flow planes) in comparison with that of Fig. 10. On the cylindrical afterbody, three cross-flow planes are only shown on a portion of its length. It should be noted here that slight flow unsteadiness has been detected during the computations. In Fig. 12, two snap shots are shown for the unsteady asymmetric flow solution of the conecylinder configuration of $1: 2$. Since the time step for a stable time-accurate solution of this case does not exceed $10^{-5}$, it is computationally prohibitive to fully solve this case.

Next, we show a comparison of the computed results with available experimental data. For this purpose, we consider the cone-cylinder configuration of $0.5: 0.5$ which was experimentally tested by Landrum ${ }^{8}$. The configuration angle of attack is $46.1^{\circ}$, the Mach number is 1.6 and the Reynolds number based on the total configuration length (cone + cylinder) is $6.6 \times 10^{6}$. The cone semi-apex angle is $9.5^{\circ}$. The problem is solved using a grid size of $161 \times 81 \times 65$. Figure 13 shows the surfacepressure coefficient along with the experimental data, the total-pressure-loss contours and the total Mach-number contours at the axial stations of $0.075,0.125,0.225$, $0.475,0.575$ and 0.775 . First, the computed and measured surface-pressure coefficient are in good agreement on all the axial stations. Second, by studying the totalpressure-loss contours along with the total Mach-number contours, it is seen that flow asymmetry starts slightly at $X / L=0.0750$, and spatially grows in the downstream direction. Moreover, the asymmetry changes sides in the downstream direction. This comparison conclusively validates our computed results and the grid size.

\section{Concluding Remarks}

The unsteady, compressible, thin-layer Navier-Stokes equations are used to obtain three-dimensional, asymmetric, vortex-flow solutions around cones and cone-cylinder configurations. Several important issues are addressed in the present study. By increasing the flow Reynolds number for flows around a cone, we have shown that the flow asymmetry becomes strong and changes sides in the downstream direction. For the high-Reynolds flows, the spatial asymmetric flow develops in a wavy manner, which is qualitatively similar to the temporal asymmetric flow development of the locally-conical solutions, where the flow asymmetry develops in a periodic manner. By adding a cylindrical afterbody to the conical forebody, the flow asymmetry becomes stronger in comparison with that of the isolated cone. As the length of the cylindrical afterbody is increased, the flow asymmetry becomes stronger and unsteady. All these flow solutions have been obtained by using a short-duration side-slip disturbance. Finally, the computed results and grid used are conclusively validated.

\section{Acknowledgement}

This research work is supported by the NASA Langley Research Center under Grant No. NAG-1-994. The computations have been carried out using the CRAY 2XMP (Voyger) of the NASA Langley Research Center.

\section{References}

1. Kandil, O. A., Wong, T-C. and Liu, C. H., "Prediction of Steady and Unsteady Asymmetric Vortical Flow Around Cones," AIAA 90-0598, January 1990. Also to appear in AIAA Joumal, Vol. 29, NO. 8, 1991.

2. Kandil, O. A., Wong, T-C. and Liu, C. H., "Asymmetric Flow Around Cones with Noncircular Sections," AGARD Symposium on Missile Aerodynamics, AGARD CP No. 493, Friedrickshafen, Germany, October 1990, pp. 16.1-16.11.

3. Kandil, O. A., Wong, T-C. and Liu, C. H., "Numerical Simulation of Steady and Unsteady Asymmetric Vortical Flows," ASME Symposium on Non-Steady Fluid Dynamics, FED-Vol. 92, Toronto, Canada, June 1990, pp. 99-108. To appear in the Joumal of Fluids and Structures, 1991.

4. Kandil, O. A., Wong, T-C., Kandil, H., A. and Liu, C. H., "Computation and Control of Asymmetric Vortex Flow Around Circular Cones Using Navier-Stokes Equations," ICAS Paper No. 3.5.3, Vol. 2. Stockholm, Sweden, September 1990, pp. 883-893.

5. Kandil, O. A., Wong, T-C., Kandil, H. A. and Liu, C. H., "Thin-Layer and Full Navier-Stokes, LocallyConical and Three-Dimensional Asymmetric Solutions," AIAA 91-0547, January 1991.

6. Degani, D. and Schiff, L. B., "Numerical Simulation of the Effect of Spatial Disturbance on Vortex Asymmetry," AIAA Paper 89-0340, January 1989.

7. Degani, D., "Numerical Investigation of the Origin of Vortex Asymmetry," AIAA 90-0593, January 1990. 
8. Landrum, E. J., "Wind-Tunnel Pressure Data at Mach Numbers from 1.6 to 4.63 for a Series of Bodies of Revolution at Angles of Attack from $-4^{\circ}$ to $60^{\circ}$," NASA Technical Memorandum, NASA TM-X-3558, October 1977.

9. Peake, D. J., Fisher, D. f. and McRae, D. S., "Flight Wind Tunnel and Numerical Experiments with a Slender Cone at Incidence," AIAA Journal, Vol. 20, No. 10, October 1979, pp. 1338-1345.

10. Lamont, P. J., "Pressure Around an Inclined Ogive Cylinder with Laminar, Transitional, or Turbulent Separation," AIAA Joumal, Vol. 20, No. 11, November 1980, pp. 1492-1499.

11. Lamont, P. J., "The Complex Asymmetric Flow Over a 3.5D Ogive Nose and Cylindrical Afterbody at High
Angles of Attack," AIAA Paper 82-0053, January 1982.

12. Yanta, W. J. and Wardlaw Jr., "The Secondary Separated Region on a Body at High Angles-of-Attack," AIAA Paper 82-0343, January 1982.

13. Keener, E. R. and Chapman, G. R., "Similarity in Vortex Asymmetrics Over Slender Bodies and Wings," AIAA Journal, Vol. 15, No. 9, September 1977, pp. 1370-1372.

14. Rediniotis, O., Stapountzis, H. and Telinois, D. P., "Vortex Shedding Over Nonparallel Edges," VPI\&SU Engineering Report, VPI-E-88-39, December 1988.

15. Zilliac, G., Degani, D. and Tobak, "Asymmetric Vortices on a Slender Body of Revolution," AIAA 90-0388, January 1990. 


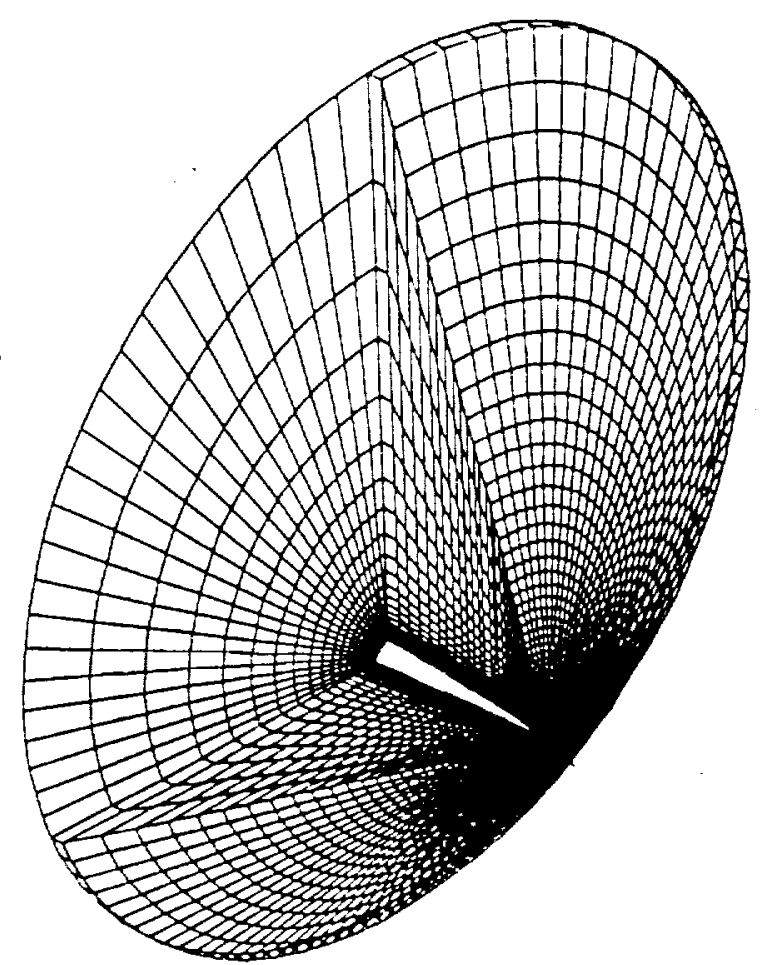

Figure 1. Typical conical grid for a three-dimensional cone, $161 \times 81 \times 65$.
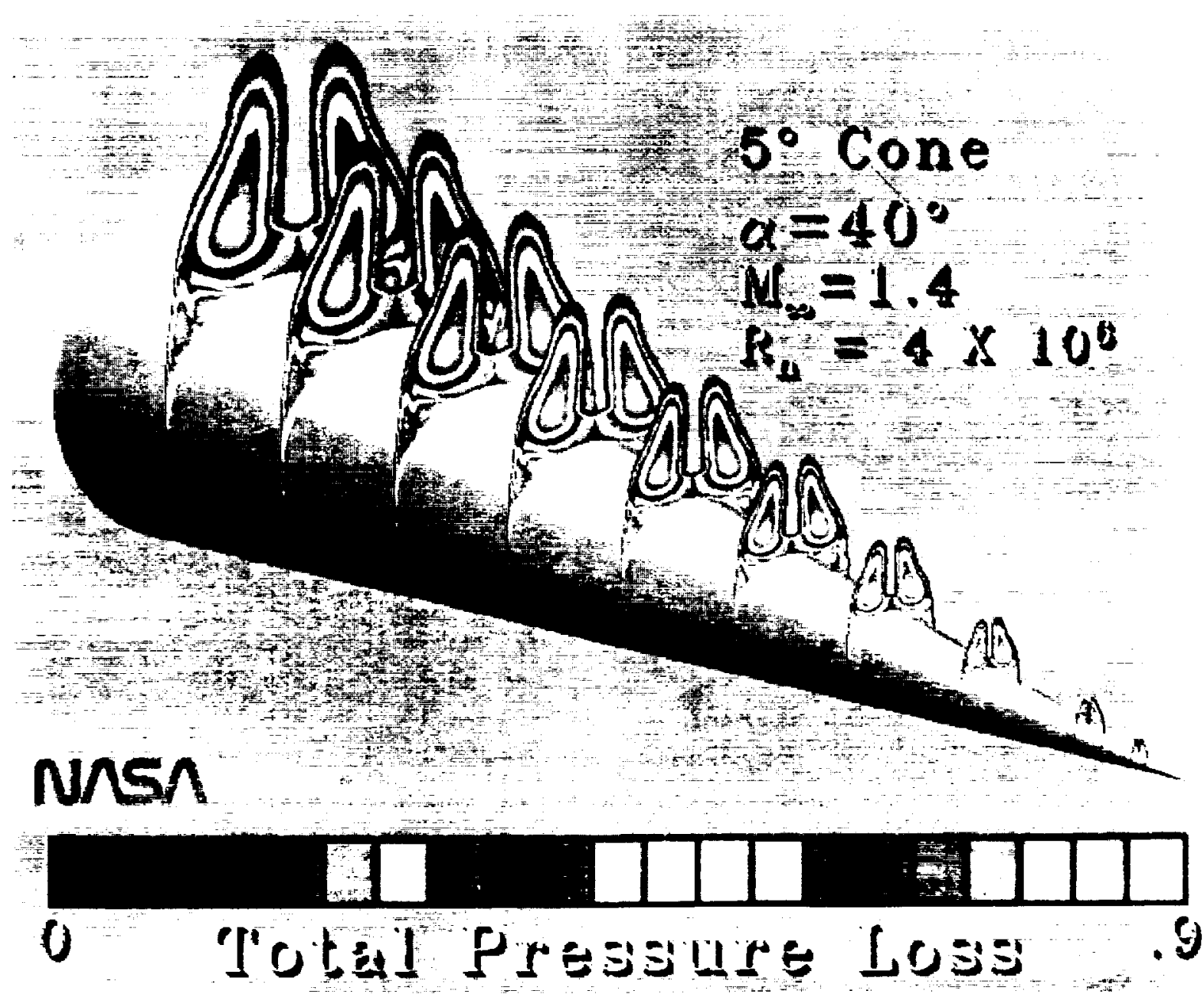

Figure 2. Asymmetric flow solution around a cone of unit length, short-duration side slip. 


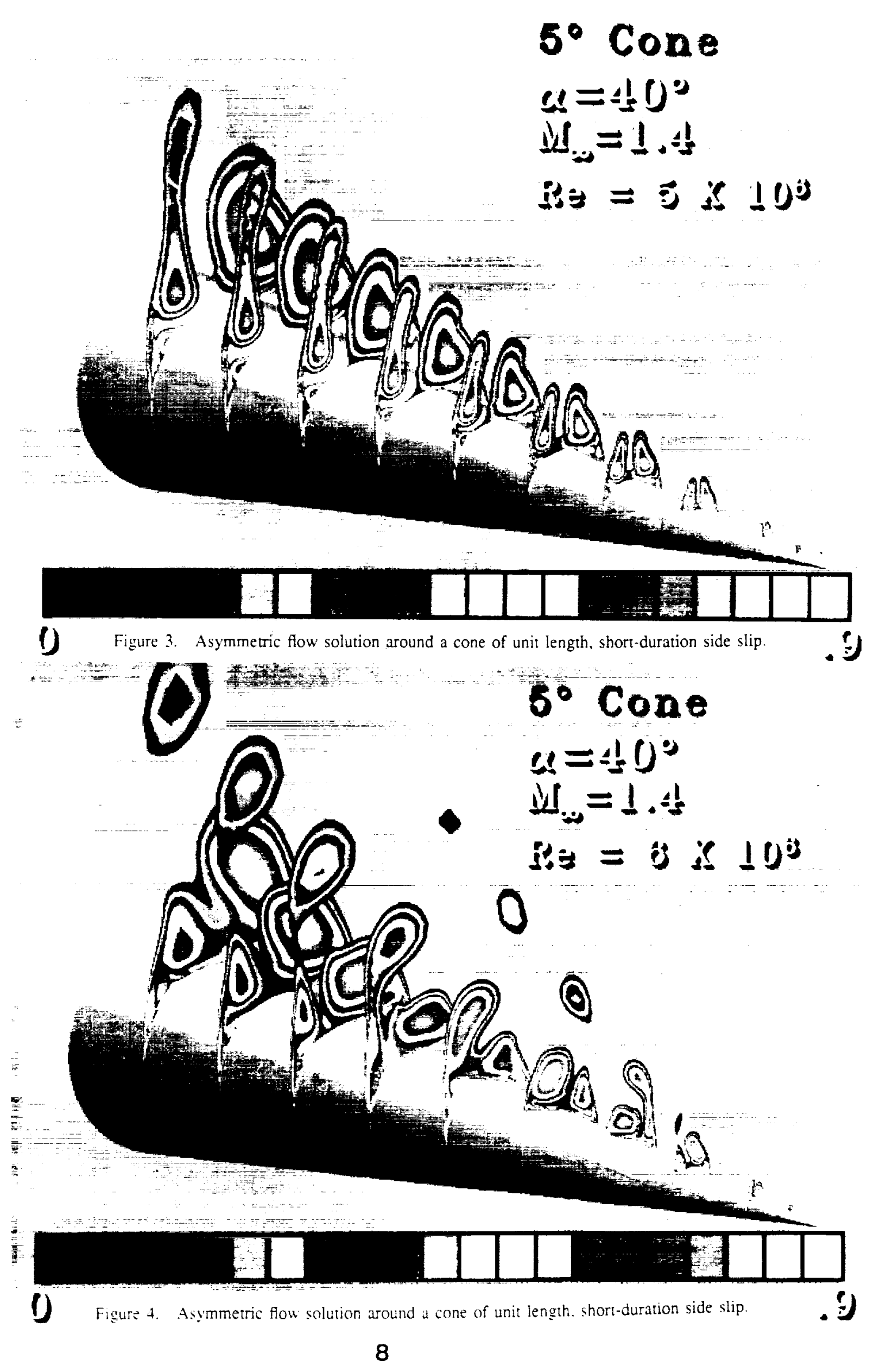



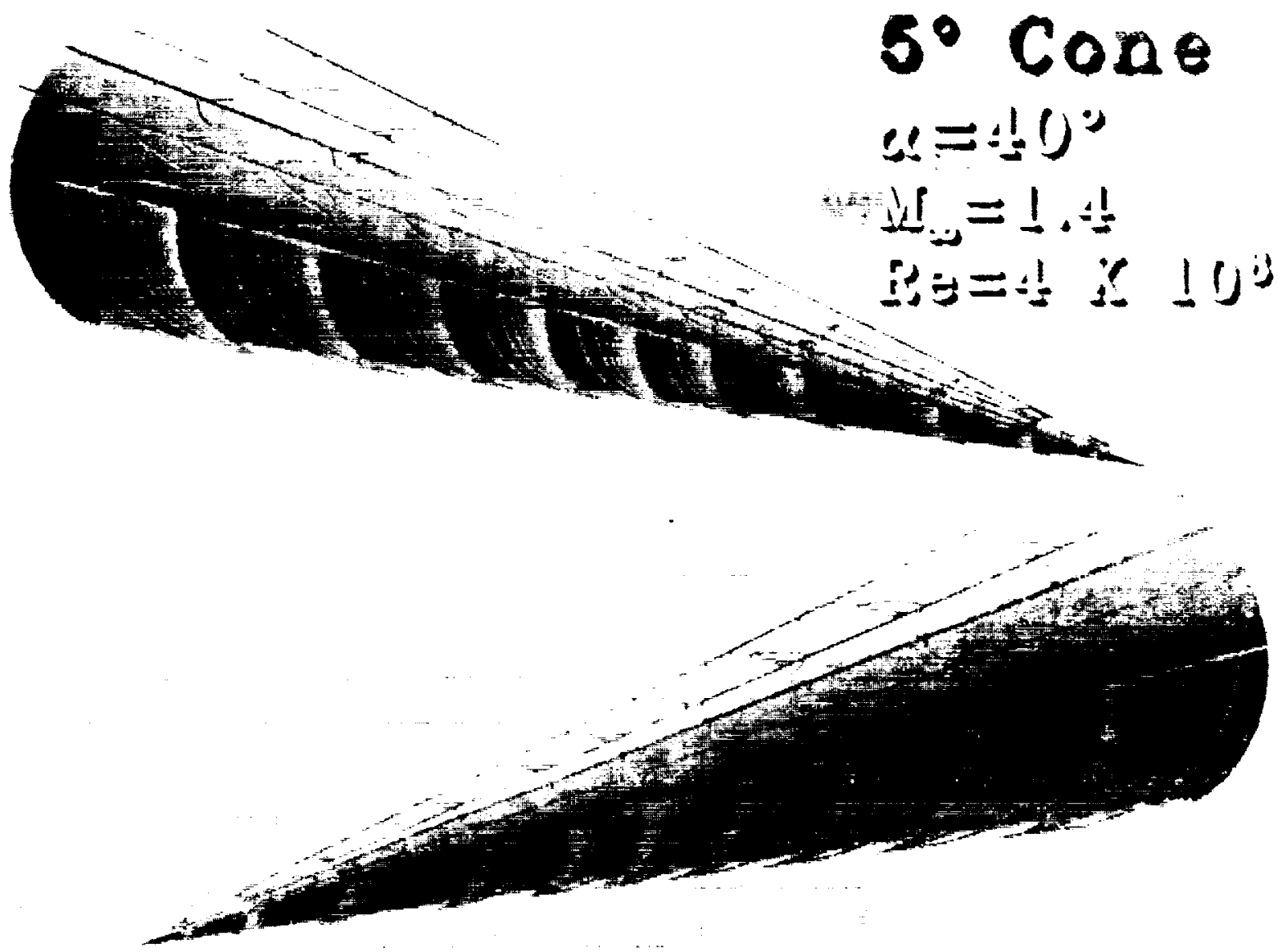

Figure 5. Front and rear sideviews of the limiting streamlines.

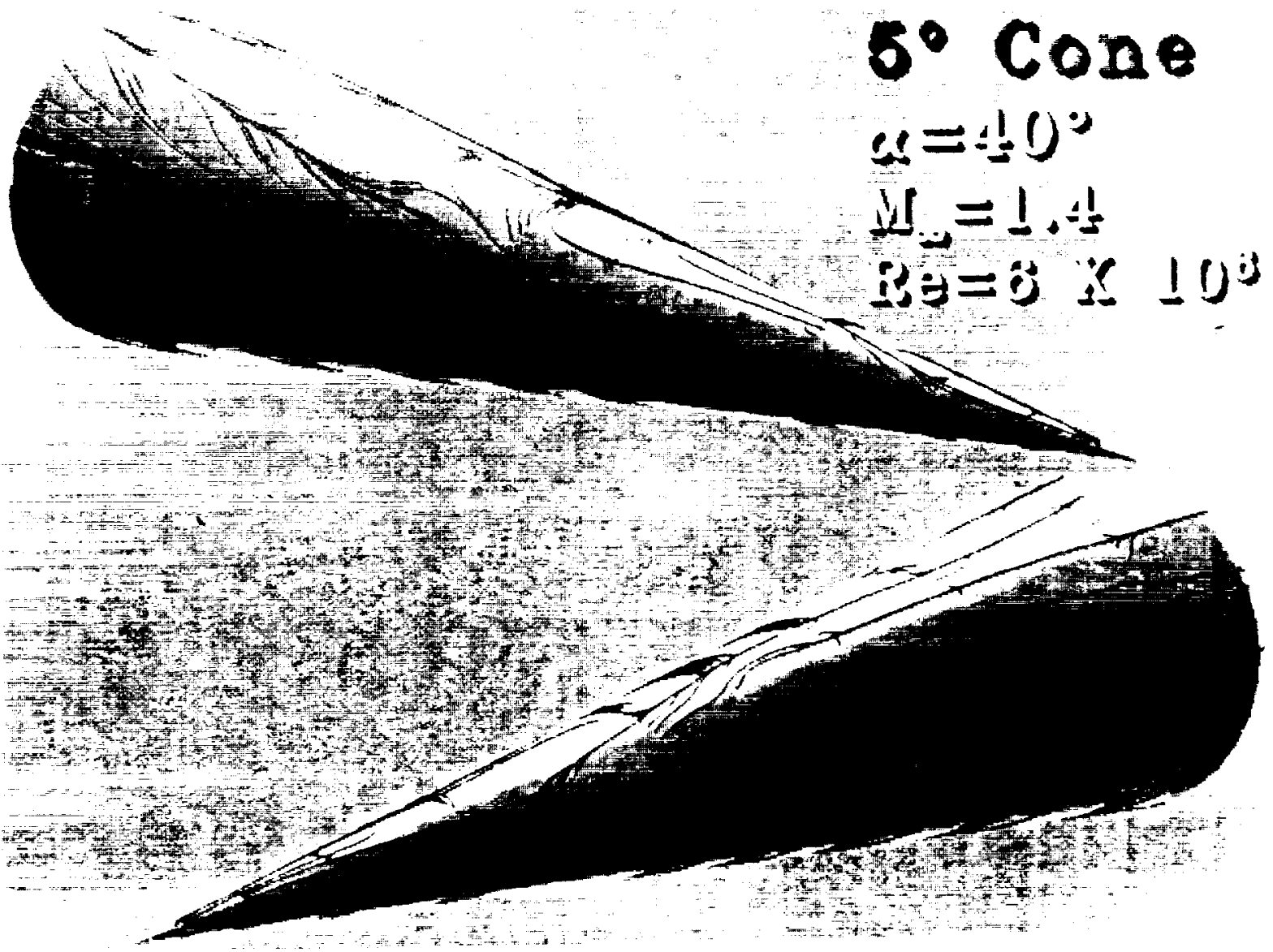

Figure 6. Front and rear sideviews of the limiting streamlines. 


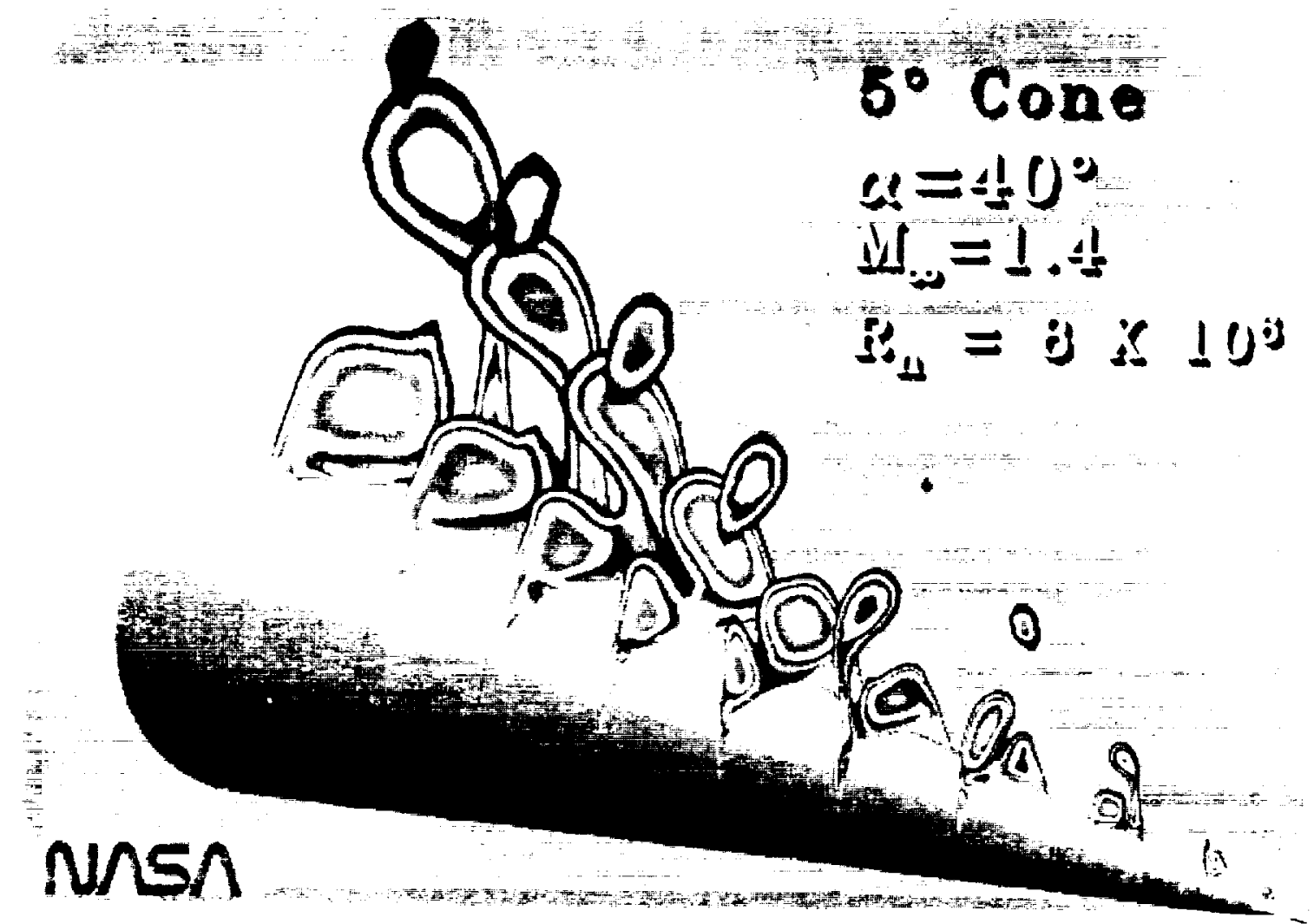

Figure 7. Asymmetric flow solution around a cone of unit length, short-duration side slip.

\section{Locally-Conical Flow}

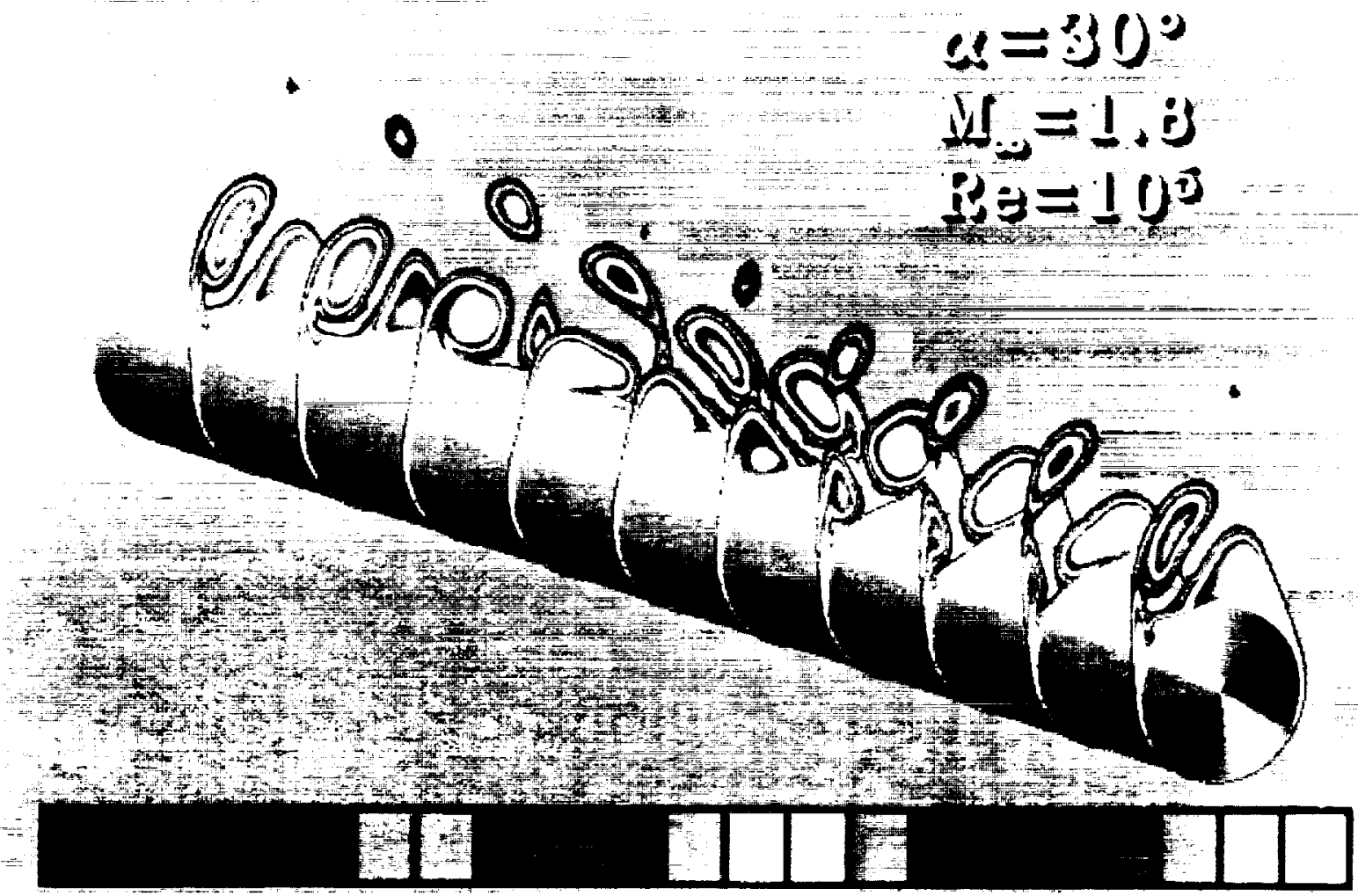

Digure 8. Unsteady asymmetric locally-conical flow solution at different time steps within one
cycle (cylinder axis is a time axis), $\lrcorner t=10^{-3}$. 


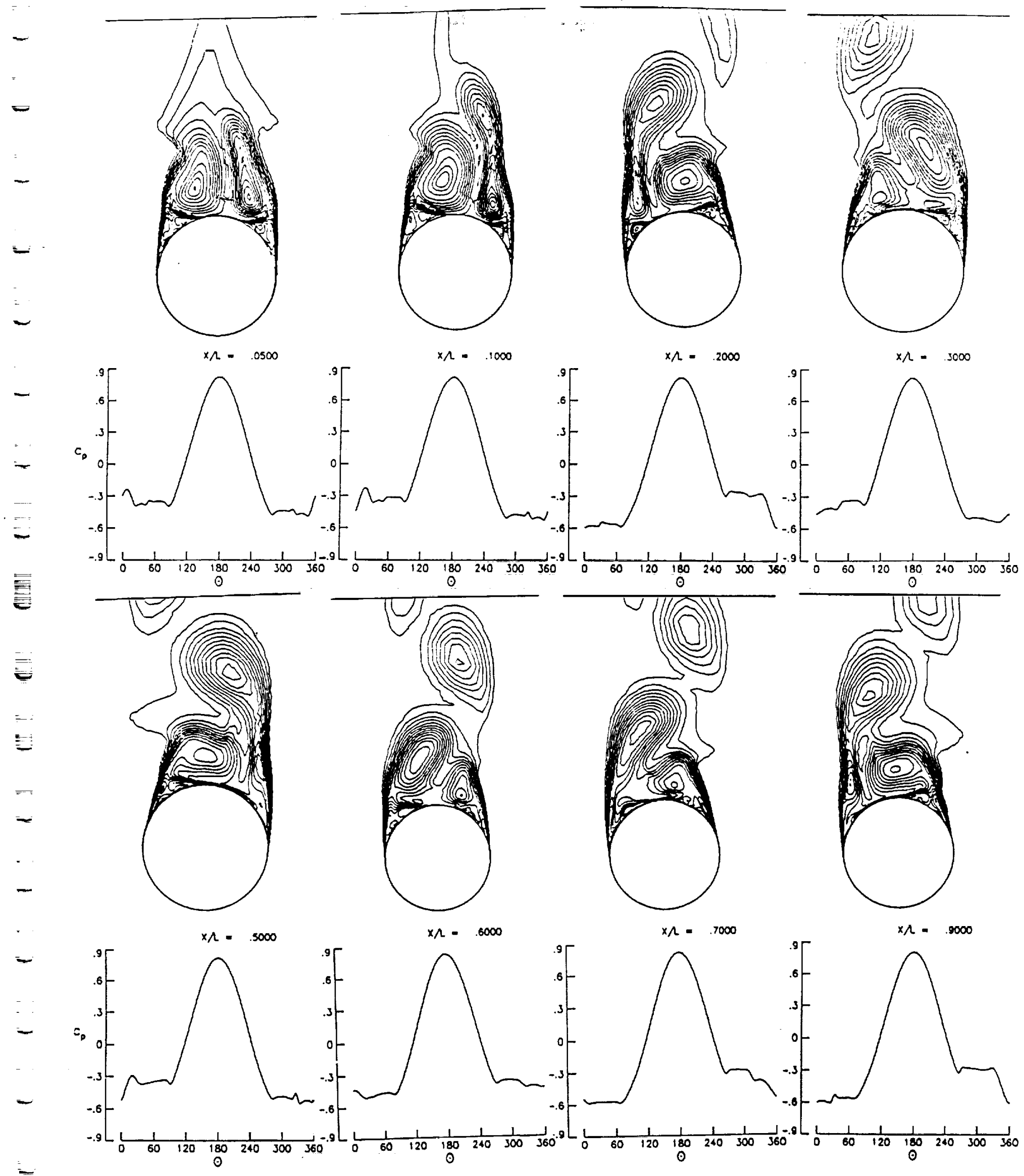

Figure 9. Total-pressure-loss contours and surface-pressure coefficient at different axial statons, a cone of unit length, $\alpha=40^{\circ}, \mathrm{M}_{\infty}=1.4, \mathrm{R}_{\mathrm{e}}=8 \times 10^{6}$.

11 

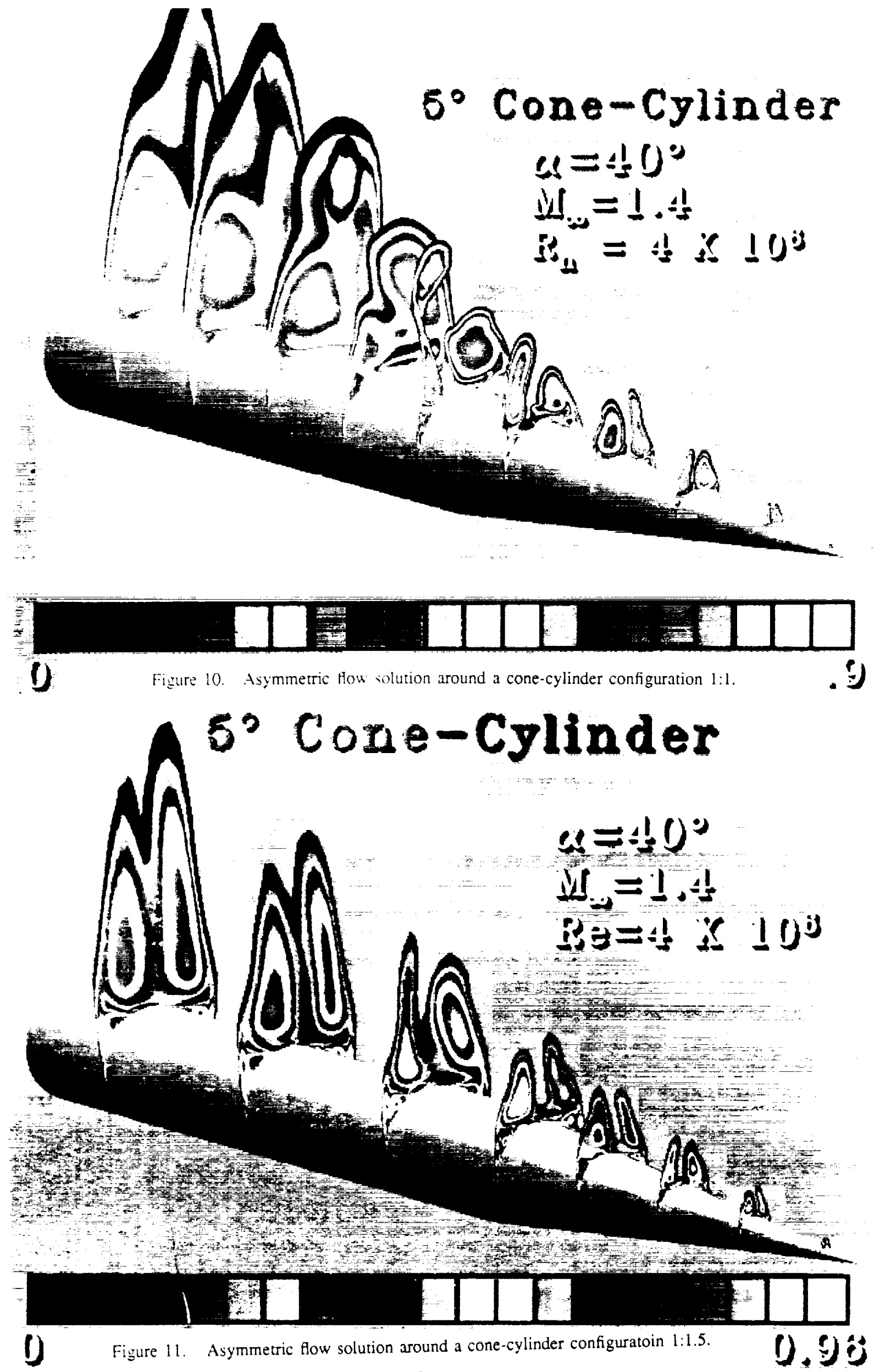


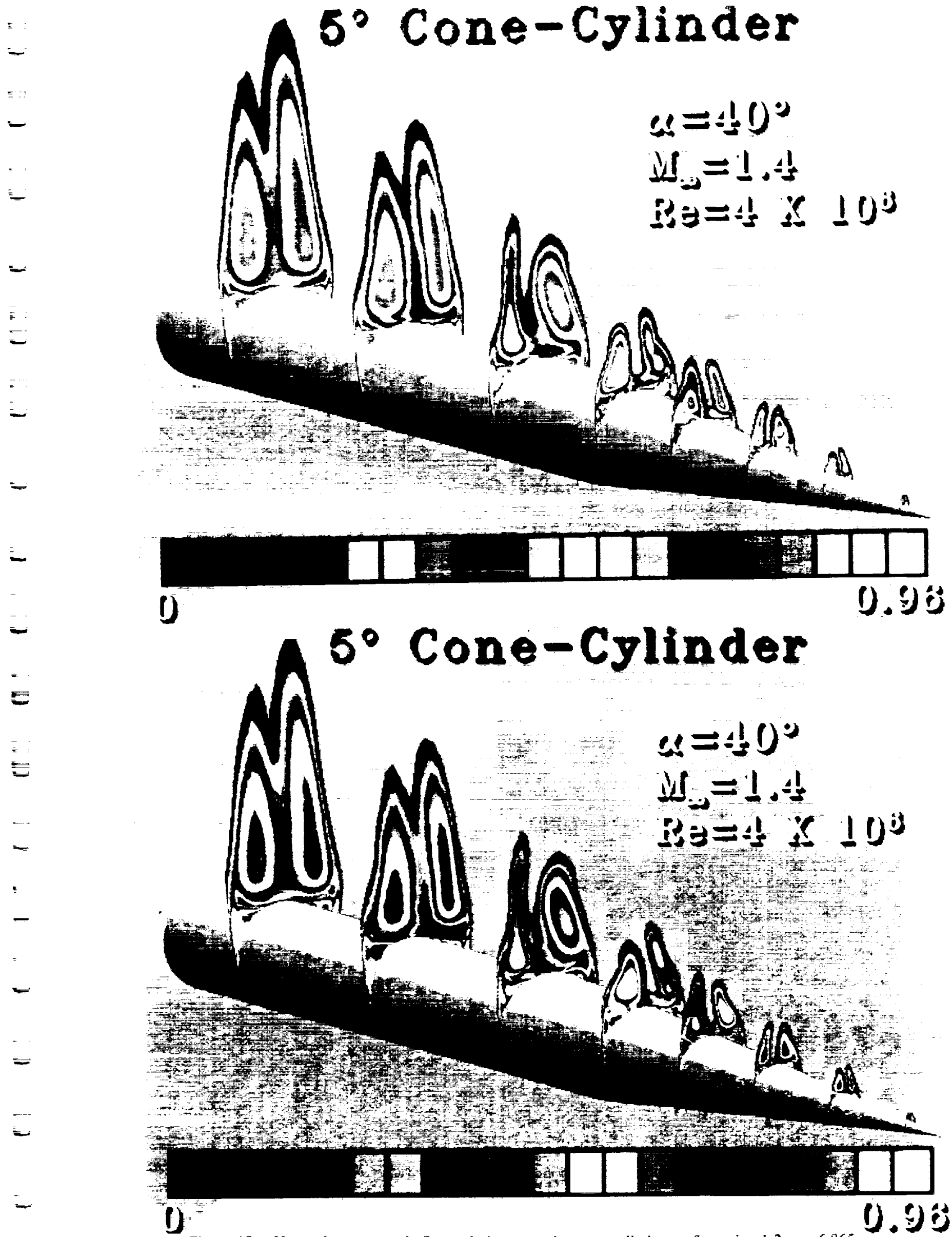

Figure 12. Unsteady asymmetric flow solution around a cone-cylinder configuration $1: 2, n=6,865$; $9767, \Delta t=10^{-5}$. 

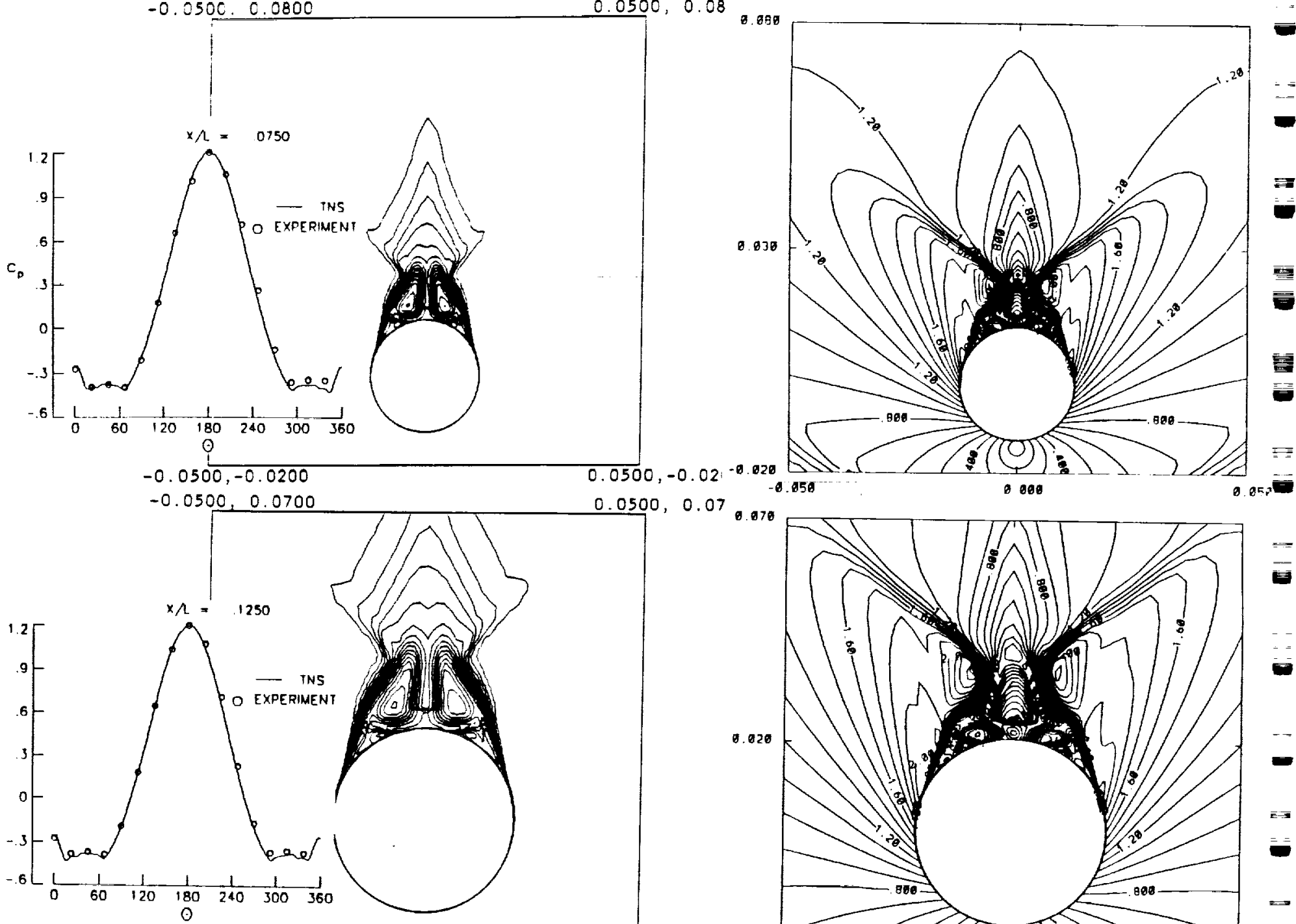

$0.0500,0.07$
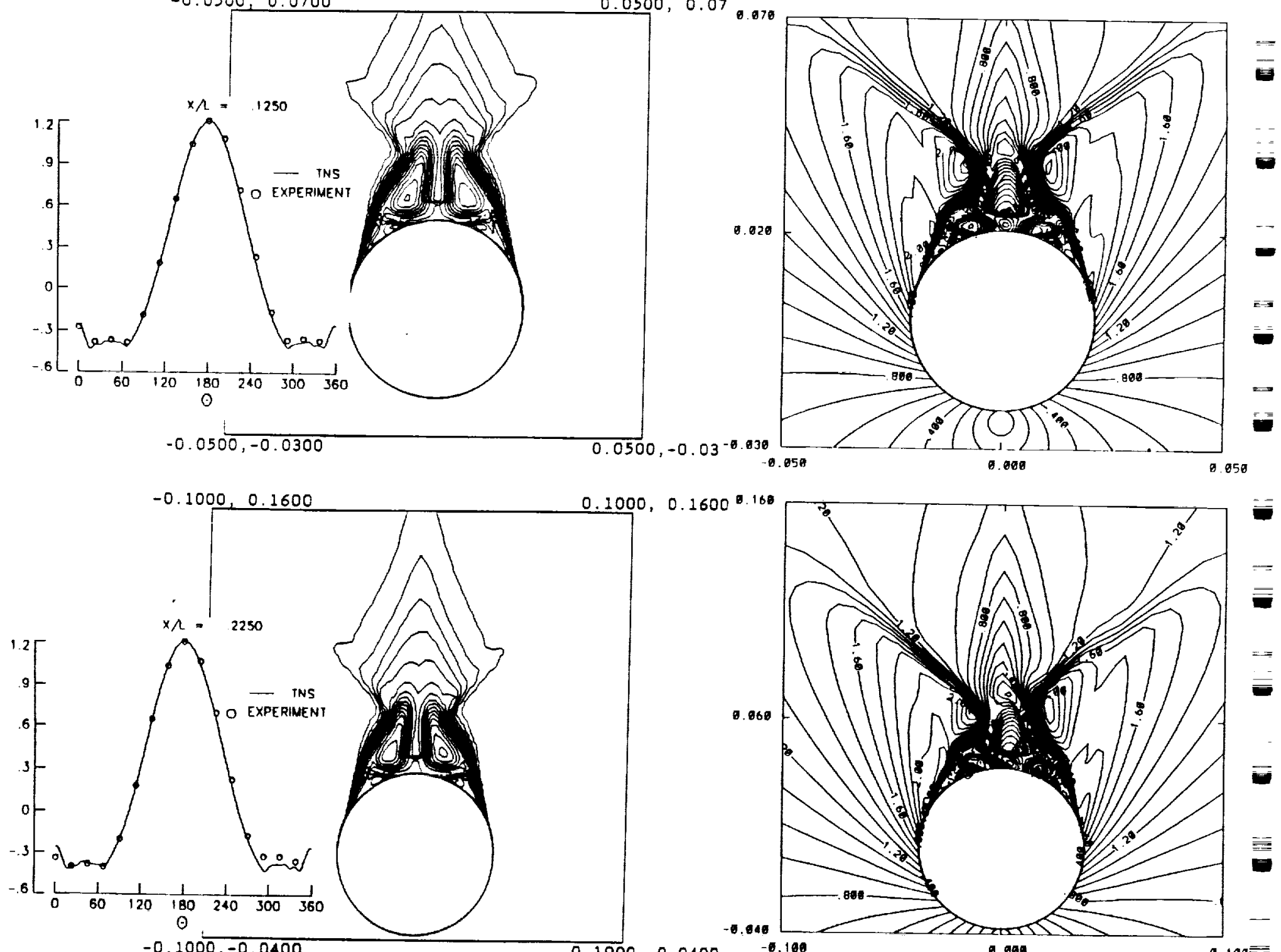

$0.0500,-0.03$

$-0.258$

$a$

05

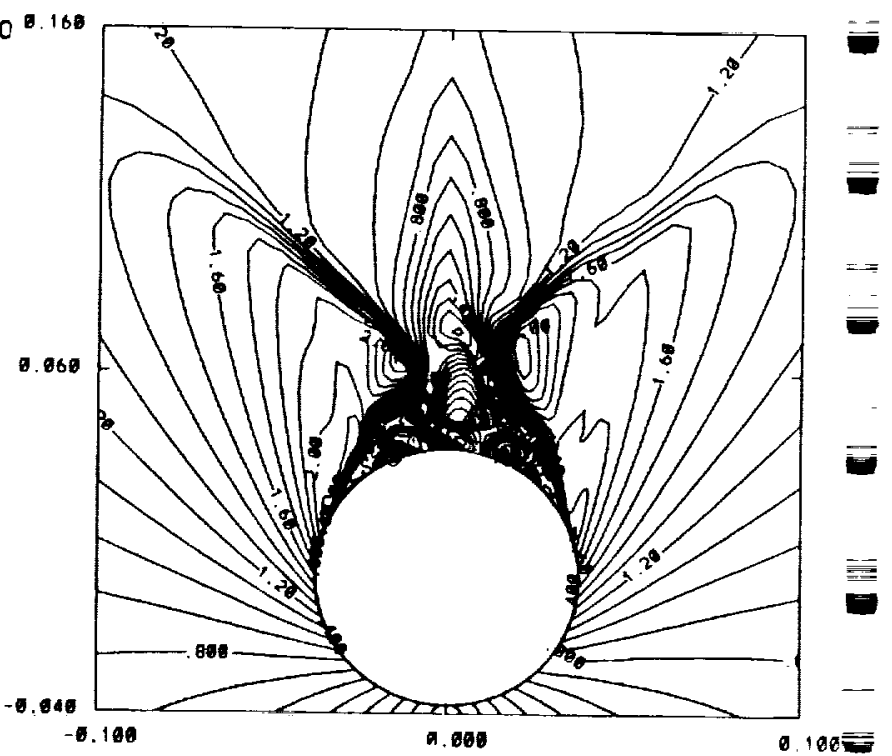

$0.1000,-0.0400$

$0.1000,0.1600$ 

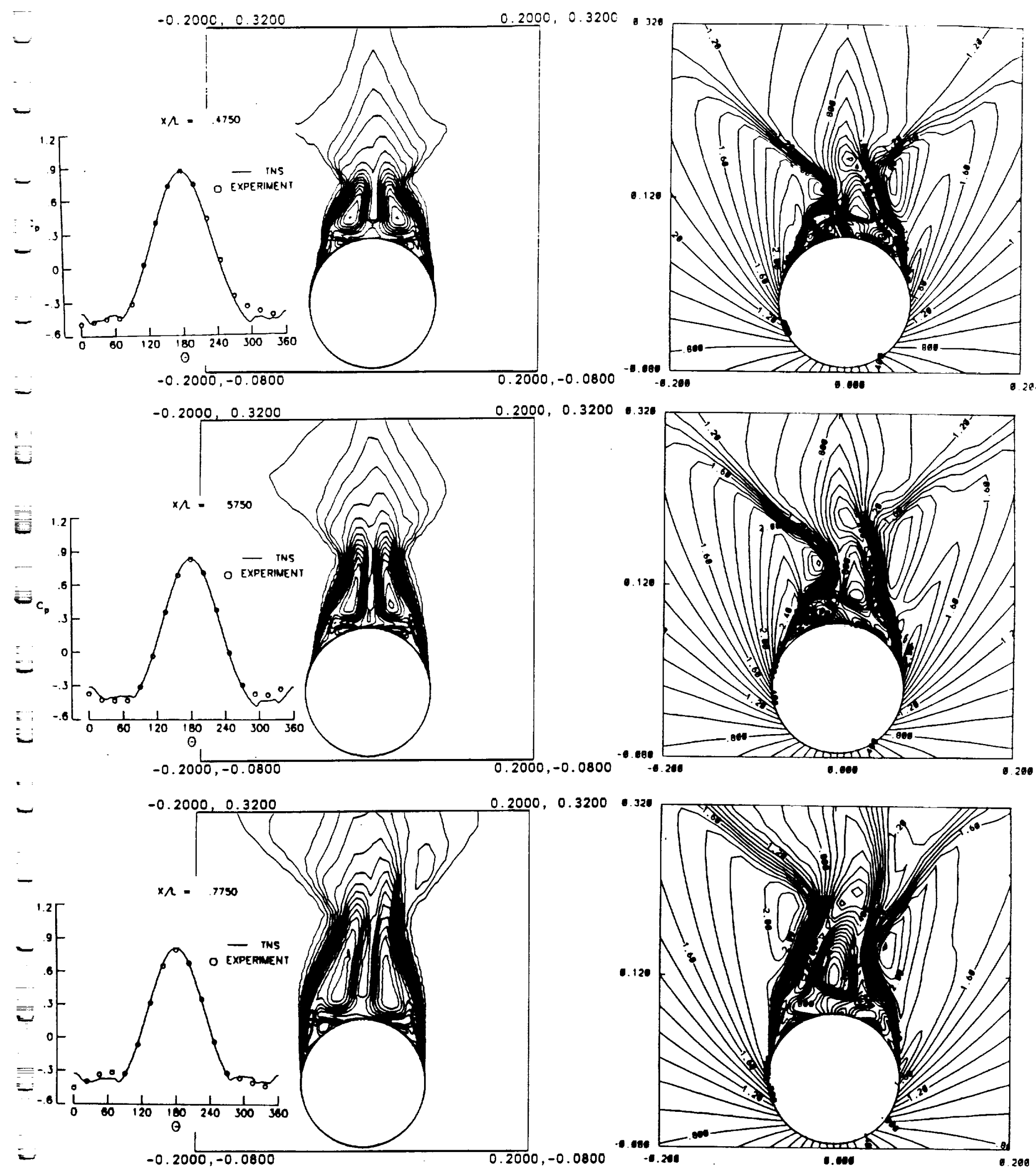

Figure 13. Surface pressure, total-pressure-loss and Mach contours at different axial stations, conecylinder configuration 1:1, $\alpha=46.1^{\circ}, \mathrm{M}_{\infty}=1.6, \mathrm{R}_{e}=6.6 \times 10^{6}$, comparison with experimental data (Ref. 8). 
